\title{
Fabrication of Axial and Radial Heterostructures for Semiconductor Nanowires by Using Selective-Area Metal-Organic Vapor-Phase Epitaxy
}

\author{
K. Hiruma, K. Tomioka, P. Mohan, L. Yang, J. Noborisaka, B. Hua, A. Hayashida, \\ S. Fujisawa, S. Hara, J. Motohisa, and T. Fukui
}

Research Center for Integrated Quantum Electronics, Hokkaido University, North 8, West 13, Sapporo 060-8628, Japan

Correspondence should be addressed to T. Fukui, fukui@rciqe.hokudai.ac.jp

Received 30 April 2011; Accepted 10 August 2011

Academic Editor: Anna Fontcuberta I. Morral

Copyright (C) 2012 K. Hiruma et al. This is an open access article distributed under the Creative Commons Attribution License, which permits unrestricted use, distribution, and reproduction in any medium, provided the original work is properly cited.

\begin{abstract}
The fabrication of GaAs- and InP-based III-V semiconductor nanowires with axial/radial heterostructures by using selective-area metal-organic vapor-phase epitaxy is reviewed. Nanowires, with a diameter of 50-300 nm and with a length of up to $10 \mu \mathrm{m}$, have been grown along the $\langle 111\rangle \mathrm{B}$ or $\langle 111\rangle$ A crystallographic orientation from lithography-defined $\mathrm{SiO}_{2}$ mask openings on a group IIIV semiconductor substrate surface. An InGaAs quantum well (QW) in GaAs/InGaAs nanowires and a GaAs QW in GaAs/AlGaAs or GaAs/GaAsP nanowires have been fabricated for the axial heterostructures to investigate photoluminescence spectra from QWs with various thicknesses. Transmission electron microscopy combined with energy dispersive X-ray spectroscopy measurements have been used to analyze the crystal structure and the atomic composition profile for the nanowires. GaAs/AlGaAs, InP/InAs/InP, and $\mathrm{GaAs} / \mathrm{GaAsP}$ core-shell structures have been found to be effective for the radial heterostructures to increase photoluminescence intensity and have enabled laser emissions from a single GaAs/GaAsP nanowire waveguide. The results have indicated that the coreshell structure is indispensable for surface passivation and practical use of nanowire optoelectronics devices.
\end{abstract}

\section{Introduction}

Interest in semiconductor nanowires has been growing over the past decade when the fabrication technology for Si-integrated circuits entered an advanced phase where the gate length of a field effect transistor (FET) shrank to a few tens of nanometers with the evolution of the technology. Fabrication of nanostructures to explore nanophotonics has also been a focus of interest for those who have been pursuing a single-photon source that can be used in optical communication systems that are expected to provide high degrees of reliability. Etching of semiconductor crystals has been widely used in conventional production processes to fabricate FETs and optical devices. However, eliminating damage or contamination created by etching on the crystal surface has become more difficult as device sizes have reduced. Compared to etching-based top-down methods of fabrication, the bottom-up method based on crystal growth is a counter approach to fabricating nanostructures without any concern for damage during fabrication.

Wagner and Ellis reported the vapor-liquid-solid (VLS) growth of Si whiskers using Au as a catalyst [1] to form semiconductor nanowires in 1964. Since then, various nanowires comprised of $\mathrm{Si}[2,3]$, Ge [2, 3], GaAs [4], InP [5], $\mathrm{In}_{2} \mathrm{O}_{3}$ [6], $\mathrm{GaN}$ [7], and $\mathrm{ZnO}$ [8] grown by VLS have been reported and the crystal structure of the nanowires has been analyzed and applications to light emitting diodes (LEDs) [9], gas sensors [10], piezoelectric generators [11], solar cells [12], resonant tunneling diodes [13], and FETs [14] have been discussed. When we fabricate nanostructures by VLS, we do not always need lithography. So the method has been popular for those who are interested in growth mechanism, nanostructure physics, and novel functional devices. However, incorporating catalysts into the grown crystal might be a concern in using the VLS method. Au, when incorporated into $\mathrm{Si}$, is known to form a mid-gap impurity level and acts as 
a carrier trap that might degrade the performance of devices [15]. Therefore, if the performance or reliability of devices is pursued, the VLS growth process should be investigated with consideration whether or not catalysts form a carrier trap level in semiconductors.

Nanowires formed by VLS using a self-catalyst, that is, a $\mathrm{Ga}$ or In droplet, which is, thought to be consumed as a source of crystal growth, have been reported for GaAs/AlGaAs core-shell and GaAs/InGaAs axial heterostructures in molecular beam epitaxy $[16,17]$. Si and Ge nanowires grown from a thin $\mathrm{SiO}_{2}$ layer without using a metal catalyst have also been reported [18]. The methods are based on the VLS mechanism but have no concern over contamination originated from catalyst materials that are not consumed for growth.

The formation of nanowires by selective catalyst-free growth using a mask pattern and columnar growth based on anisotropy of the crystal structure with wurtzite have been reported since the late 1990s. These methods are different from the VLS, have not needed any catalysts, and there have been no concerns over contamination by them. Hamano et al. reported GaAs pillar (nanowire) arrays selectively grown along the $\langle 111\rangle \mathrm{B}$ axial direction by using metal organic vapor phase epitaxy (MOVPE) on a GaAs (111)B substrate with lithography-defined $\mathrm{SiO}_{2}$ mask openings [19]. They discussed the GaAs growth rate along $\langle 111\rangle \mathrm{B}$ as a function of the pillar radius and the gas supply pressure of an $\mathrm{AsH}_{3}$ source. Motohisa et al. investigated the diameter dispersion of GaAs nanowire arrays selectively grown by MOVPE in an effort to fabricate a photonic crystal lattice [20]. Yoshizawa et al. reported $\mathrm{GaN}$ nanocolumn growth on an $\mathrm{Al}_{2} \mathrm{O}_{3}(0001)$ surface during RF radical-source molecular beam epitaxy (RF-MBE) [21, 22]. GaN nanocolumns as thin as $50 \mathrm{~nm}$ and as long as $600 \mathrm{~nm}$ have been obtained without using a mask pattern. The methods of growth later evolved into fabricating $\mathrm{GaN} / \mathrm{InGaN}$ nanocolumn arrays grown using a lithographydefined mask pattern for red-green-blue or multicolor LEDs in the visible wavelength range [23]. $\mathrm{ZnO}$ nanorods, as thin as $25 \mathrm{~nm}$, grown perpendicular to an $\mathrm{Al}_{2} \mathrm{O}_{3}(00 \cdot 1)$ surface without using a mask pattern during MOVPE have been reported by Park et al. [24]. Using this technology, $\mathrm{ZnO}$ nanorods formed vertically on the top layer of a GaN LED have been demonstrated to enhance the efficiency with which light output is extracted from devices [25].

Paetzelt et al. have recently reported selective-area growth of GaAs and InAs nanowires on a GaAs(111)B surface with $\mathrm{SiN}_{x}$ templates by using MOVPE [26]. They argued for the dependence of the rate of nanowire growth on the growth conditions and nanowire radius/pitch. InAs/GaAs heterostructures have also been grown to investigate how the shape and crystal structure of nanowires change. Sladek et al. reported GaAs and AlGaAs nanowires selectively grown on a GaAs(111)B substrate with a hydrogen-silsesquioxane mask pattern by MOVPE using $\mathrm{N}_{2}$ carrier gas in an effort to fabricate a conductive channel in modulationdoped GaAs/AlGaAs heterostructure nanowires [27]. They observed that the lateral growth of GaAs nanowires had increased due to the reduced surface diffusion of growth species on the mask when $\mathrm{Si}_{2} \mathrm{H}_{6}$ was added to the source gases, or due to polycrystalline layer growth on the mask when GaAs/AlGaAs core-shell nanowires were grown.

We have concentrated on the selective-area growth of group III-V semiconductor nanowire arrays by using MOVPE over the past several years. GaAs/InGaAs axial (vertical) heterostructures [28], GaAs/AlGaAs radial (lateral) [29] and axial heterostructures [30], an InAs quantum tube sandwiched by InP [31], and GaAs/GaAsP radial/axial heterostructures [32, 33] have been fabricated to characterize crystal structures, atomic composition profiles, and photoluminescence properties with quantum size effects. Laser emissions have quite recently been achieved in GaAs/GaAsP core-shell nanowires under pulsed photoexcitation [32]. We review our studies on fabricating axial and radial heterostructures obtained by selective-area MOVPE (SA-MOVPE) and discuss their growth characteristics and optical properties in this paper.

\section{Method for Nanowire Growth and Characterization}

There is a schematic of the process for growing nanowires in Figure 1. An $\mathrm{SiO}_{2}$ layer with a thickness of 20-30 nm, which was used as a mask during SA-MOVPE, was deposited on a GaAs(111)B substrate by plasma sputtering or chemical vapor deposition. An array of mask openings was fabricated for the $\mathrm{SiO}_{2}$ layer by electron beam (EB) lithography and wet chemical etching of $\mathrm{SiO}_{2}$ through an $\mathrm{EB}$ resist pattern. The diameter of the openings ranged from 20 to $1000 \mathrm{~nm}$, and the pitch was changed between 300 and $3000 \mathrm{~nm}$. The mask opening array was fabricated in a $100 \times 100-\mu \mathrm{m}$ square area, which was separated with a $100-\mu \mathrm{m}$ isolation distance by removing $\mathrm{SiO}_{2}$ from neighboring square array areas that had the same size. The shape of the opening was designed to be hexagonal in EB lithography, but it was rounded into a circular shape after wet chemical etching when the pattern size was less than $100 \mathrm{~nm}$. The source materials used for MOVPE were trimethylgallium (TMG), trimethylaluminium (TMA), trimethylindium (TMI), arsine $\left(\mathrm{AsH}_{3}: 5\right.$ or $20 \%$ in $\mathrm{H}_{2}$ ), and tertiarybutylphosphine (TBP). Silane $\left(\mathrm{SiH}_{4}: 50 \mathrm{ppm}\right.$ in $\left.\mathrm{H}_{2}\right)$ was used as the n-type dopant, and diethylzinc (DEZ) was used as the p-type dopant for the device structures. The source gases were carried by palladium diffused $\mathrm{H}_{2}$, and the flow rates were controlled by mass flow controllers. The total pressure inside the growth chamber (horizontal $\mathrm{SiO}_{2}$ tube) was maintained with a vacuum pumping system at 0.1 atm by adjusting the flow of exhaust gas from the chamber. The gas flows and the total pressure inside the chamber were programmed beforehand and automatically operated by a computer during MOVPE. The substrate for MOVPE was placed on a thin $\mathrm{SiO}_{2}$ plate in the chamber. A graphite block beneath the $\mathrm{SiO}_{2}$ plate was heated by RF induction, and the temperature of the graphite block was monitored with a thermocouple, whose output was fed into the RF-induction controller. The growth temperatures adopted for the heterostructure nanowires were controlled between 600 and $850^{\circ} \mathrm{C}$. For example, when an axial heterostructure $\mathrm{GaAs} / \mathrm{GaAsP}$ was grown, the 


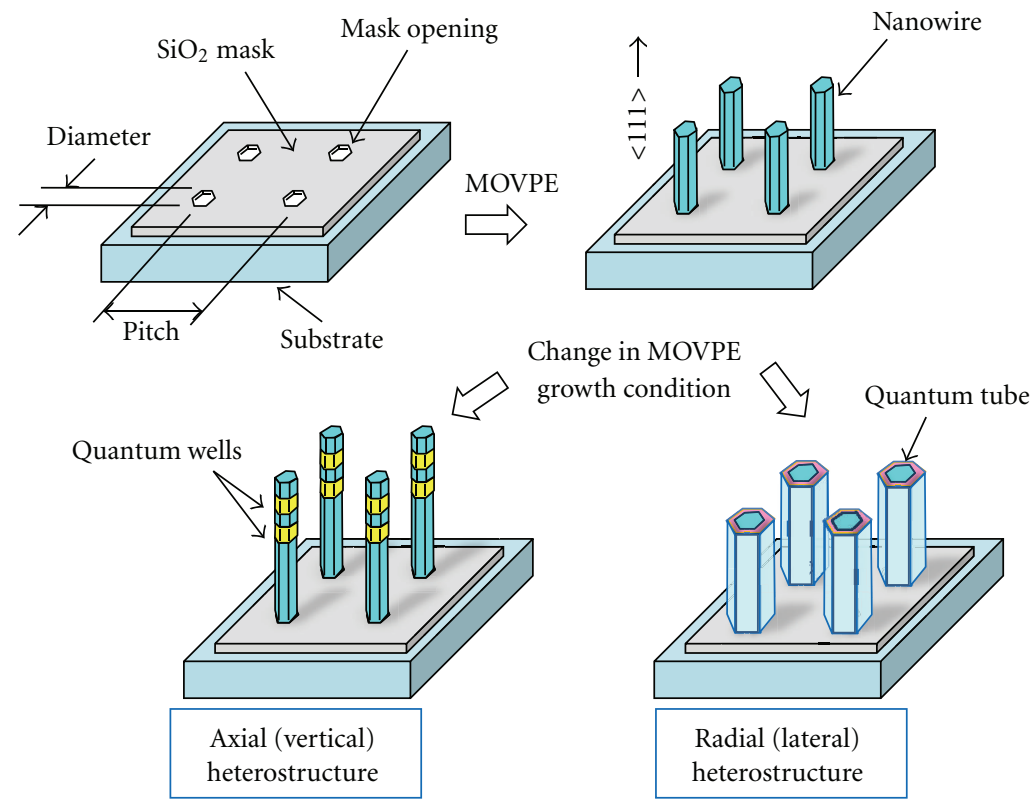

FIGURE 1: Schematic of process for forming heterostructure nanowires.

temperature was optimized to $750^{\circ} \mathrm{C}$, while, for a radial heterostructure GaAs/GaAsP, the temperatures were $750^{\circ} \mathrm{C}$ for $\mathrm{GaAs}$ and $650^{\circ} \mathrm{C}$ for GaAsP. The pressure ratio of the group III source gases to the group $\mathrm{V}$ source gases (V/III ratio) was also changed over time with the control of growth temperature.

The nanowires that were grown were observed by scanning electron microscopy (SEM) to characterize their shape, growth direction, length, and diameter. Some nanowires were further investigated by transmission electron microscopy (TEM) with energy-dispersive X-ray (EDX) spectroscopy to analyze their crystal structure and atomic composition. Photoluminescence (PL) was measured to analyze the optical properties of the nanowires. A He-Ne laser in CW operation (wavelength: $632.8 \mathrm{~nm}$ ) was used as an excitation source, and the laser light was focused to $2 \mu \mathrm{m}$ in diameter with $\times 50$ microscope objectives. We can measure PL from one nanowire or bundles of them depending on the pitch, wider or narrower than $2 \mu \mathrm{m}$. The PL from nanowire samples was guided into a monochromator (Acton SP-2300i; wavelength resolution of 0.1 or $0.4 \mathrm{~nm}$ ) and detected with a charge-coupled-device (CCD) camera, which was also used to monitor images of the sample surface.

\section{Heterostructure Growth in Axial Direction}

3.1. GaAs/InGaAs Vertical Structures with InGaAs Quantum Well [28]. The structure for the nanowires (nanopillars) with a single InGaAs/GaAs quantum well (QW) grown along the $\langle 111\rangle \mathrm{B}$ direction on the GaAs (111)B substrate surface (called sample I) is schematically shown in Figure 2(a). The growth temperature for the bottom and top GaAs barrier layers was $750^{\circ} \mathrm{C}$, their growth times were $20 \mathrm{~min}$ for the bottom and $5 \mathrm{~min}$ for the top, and the partial pressure for
$\mathrm{AsH}_{3}$ was $2.5 \times 10^{-4}$ atm and $1.3 \times 10^{-6}$ atm for TMG. The growth temperature and the growth time for the InGaAs well layer was $600^{\circ} \mathrm{C}$ and $1 \mathrm{~min}$, and the partial pressures for $\mathrm{AsH}_{3}, \mathrm{TMG}$, and TMI corresponded to $6.3 \times 10^{-5}$, $1.3 \times 10^{-6}$, and $1.2 \times 10^{-7} \mathrm{~atm}$. Under these conditions, the indium content for the InGaAs epitaxial layers grown on the GaAs (100) substrate and on the GaAs (111)B substrate were measured to be $5.5 \%$ for the former and $14.0 \%$ for the latter, which can be ascribed to fewer Ga atoms being incorporated on the GaAs (111)B substrate surface terminated with As atoms (As trimers), which are more stable at low growth temperatures, or at high As pressures [34-36]. We speculate that the As trimers impede Ga incorporation onto GaAs (111)B surface and this is why there is difference in the indium content for the InGaAs layers grown on the GaAs (100) and on the GaAs (111)B substrates. After the InGaAs well layer had been grown, a GaAs layer was grown at $600^{\circ} \mathrm{C}$ for 2 min under the same gas supply conditions as those for the bottom and top GaAs barrier layers, which were grown at $750^{\circ} \mathrm{C}$.

The nanopillars with a single InGaAs/GaAs QW fabricated on the GaAs (111)B surface were characterized by SEM (Figure 2(b)). We can see the nanopillars are hexagonal and the top and side surfaces are very smooth, which indicates that six (1-10) facets and a top (111)B facet have been formed. The standard deviation in the diameter of the nanopillars is about $8 \%$, and the standard deviation in their height is about 5\%. Compared with planar (layer) growth (thickness of about $45 \mathrm{~nm}$ ), the nanopillars are one order of magnitude taller (Figure $2(\mathrm{~d})$ ). This indicates that the nanopillar growth rate on the patterned substrate is greatly enhanced, which can be attributed to the migration of growth species containing $\mathrm{Ga}$ atoms and In atoms from the masked to the exposed region. The nanopillar growth 

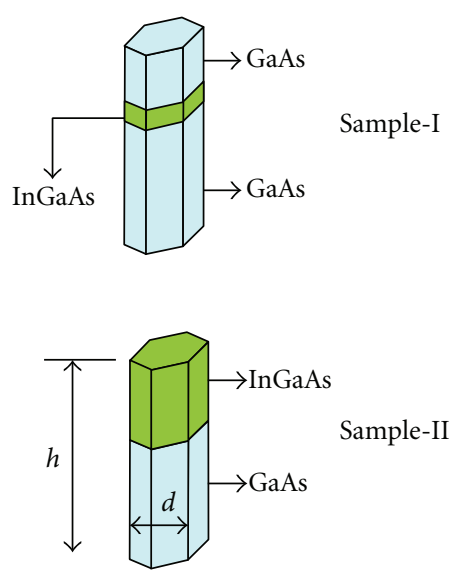

(a)

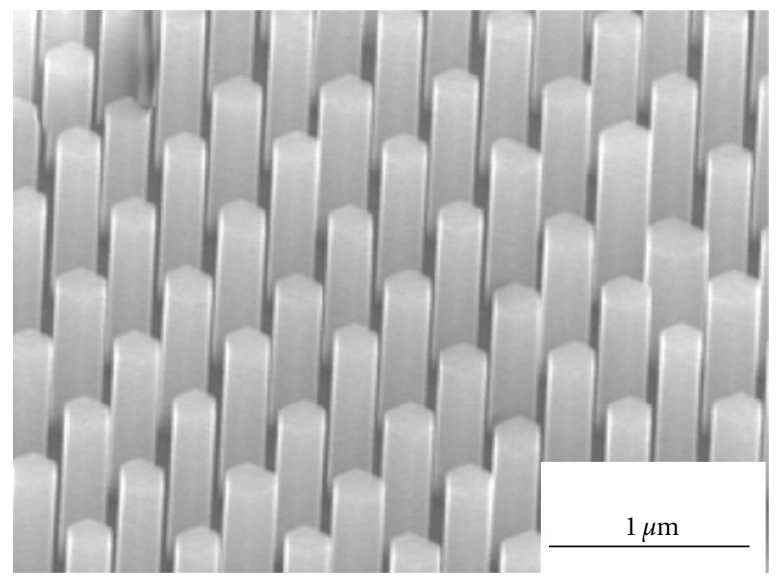

(c)

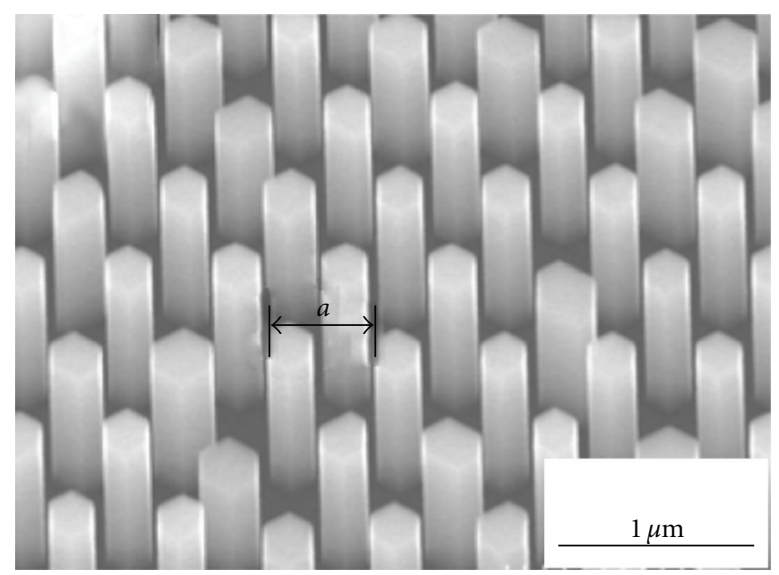

(b)

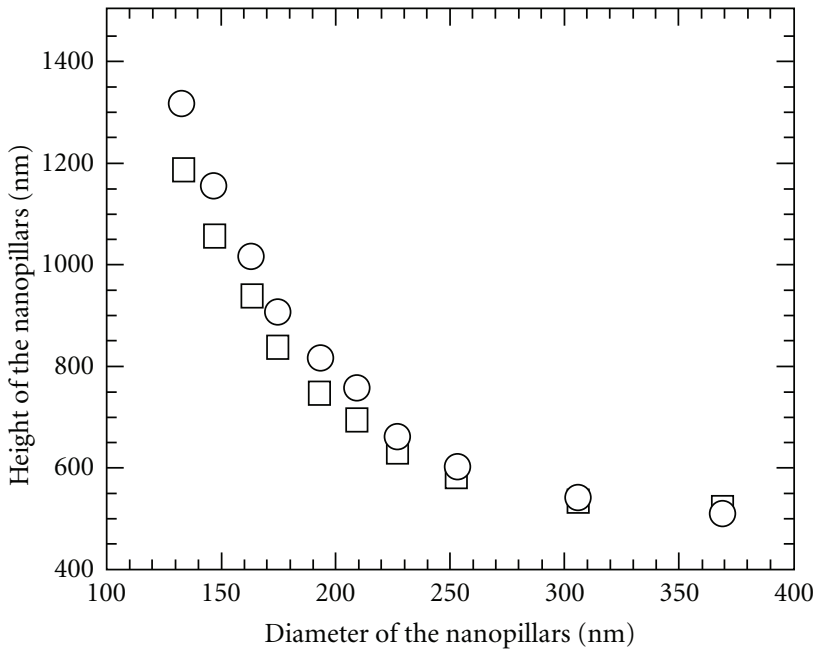

$\square$ Sample-I

O Sample-II

(d)

FIGURE 2: Structures of nanowire samples and SEM images of grown nanowires. (a) Schematics of structures for samples I and II. The nanowire diameter is defined by $d$. (b) SEM image for sample I. The nanowire pitch is defined by $a$. (c) SEM image for sample II. (d) Dependence of heights of samples I and II on their diameters [28].

rate is determined in the first approximation. We consider first the ratio of the radius of the collection region for the growth species (on the mask surface) to their diffusion lengths. Next we calculate the filling ratio in the masked pattern. The diffusion lengths for the growth species in the masked region are thought to be on the order of $1 \mu \mathrm{m}$ and longer than the pitch of the nanopillars investigated here. The filling ratio in the masked pattern is given by $(d / a)^{2}$, where $d$ and $a$ are the nanopillar diameter and the pitch, respectively, and this was constant (0.09) in our samples for various $d$ and $a$. Thus, the nanopillar growth rate was expected to be more or less independent of the pitch. However, this was not consistent with our experimental results, in which the nanopillar growth rate was larger for those with a smaller pitch. This peculiar dependence seems to be closely related to the unexpected dependence of the enhanced growth rate on pitch in the SA-MOVPE growth of GaAs nanowires that have previously been reported [37]. The explanation for the observation could be ascribed to the contribution of sidewall facets. Only the growth species on the masked region migrated to the exposed region and contributed to the initial stage of growth. The growth rate at this stage should be almost the same for all the nanopillars with different diameters. After the nanopillars were formed, six (1-10) facets appeared and started to absorb the growth species, which also migrated to the top (111)B facet and thus contributed to the growth of the nanopillars. We think that more growth species were absorbed by the six (11-0) facets as the nanopillar diameter was decreased, that is, the height of the nanopillars was increased with a decrease in the diameter even though the initial filling ratio in the masked pattern was the same.

To investigate whether the InGaAs layer had grown on the top (111)B facet or on the six (11-0) facets, only an 
InGaAs layer was grown after the initial GaAs nanopillars (called sample II) (Figure 2(a)) had formed. The growth conditions were the same as those for the bottom two layers in sample I except that the growth time for the InGaAs layer was prolonged from 1 to $5 \mathrm{~min}$. By comparing Figure 2(b) with Figure 2(c), we can see that sample I has almost the same diameter as sample II, while sample II is a little higher than sample I. The same phenomena also existed for the nanopillars with other diameters (Figure 2(d)), which indicates that the InGaAs layer had mainly grown on the top (111)B facet and the growth rate was larger than that for the GaAs layer. For example, the GaAs growth rate along the $\langle 111\rangle \mathrm{B}$ was about $45 \mathrm{~nm} / \mathrm{min}$ and the InGaAs was about $80 \mathrm{~nm} / \mathrm{min}$ for the nanopillar diameter of $130 \mathrm{~nm}$ plotted in Figure 2(d).

The micro-PL $(\mu$-PL) spectra for sample I were measured at room temperature (RT) and $77 \mathrm{~K}$. The excitation beam from a He-Ne laser (wavelength: $632.8 \mathrm{~nm}$ ) was focused to a spot of about $2 \mu \mathrm{m}$ in diameter with a $\times 50$ microscope objective and onto samples placed in a cryostat. Figure 3 has a schematic of a substrate with nanowires excited by a focused laser beam during PL measurements. The PL collected through the same microscope objective was detected with a liquid nitrogen-cooled CCD. At RT (Figure 4(a)), the peak located at about $870 \mathrm{~nm}$ originates from the band edge emission of the GaAs barrier layer, while the other peak located on the longer wavelength side originates from the first electron heavy hole excitonic recombination of the InGaAs/GaAs QW. The peak position shifts to the longer wavelength with an increase in the diameter of the nanopillars (called red shift). Red shift in the peak position can also be observed at $77 \mathrm{~K}$ (Figure 4(b)). We randomly measured the $\mu$-PL at twenty different points in the same pattern and plotted the peak positions dependent on diameter in Figure 6 to reduce the possible effects of diameter and height fluctuations in the discussion on the origin of red shift. The peak position first shifts to the longer wavelength very quickly with an increase in the diameter of the nanopillars, and the shift then slows. We can speculate that the peak position of the nanopillars will approach the band-edge emissions ( $1018 \mathrm{~nm}$ at RT and $972 \mathrm{~nm}$ at $77 \mathrm{~K}$ ) of the $\mathrm{In}_{0.14} \mathrm{Ga}_{0.86}$ As epitaxial layer grown on the GaAs (111)B substrate under the same conditions if their diameter becomes large enough. It should be noted that the shift in the peak position from the band-edge emission of the GaAs barrier layer is just $5 \mathrm{~nm}$ at RT and $3 \mathrm{~nm}$ at $77 \mathrm{~K}$ when the diameter of the nanopillars is reduced from 369 to $133 \mathrm{~nm}$, which indicates that the quantum confinement effect in the radial direction is negligible and thus does not account for the large red shift that was observed. Since the height of the nanopillars increases with a decrease in their diameter, we can naturally deduce that the thickness of the InGaAs well layer also increases correspondingly. Therefore, the quantum confinement effect in the axial direction induces the shift in the peak position to the longer wavelength and not to the shorter wavelength with an increase in the height of the nanopillars. We think that this red shift is possibly due to the stoichiometry difference in indium content for nanopillars with different diameters.

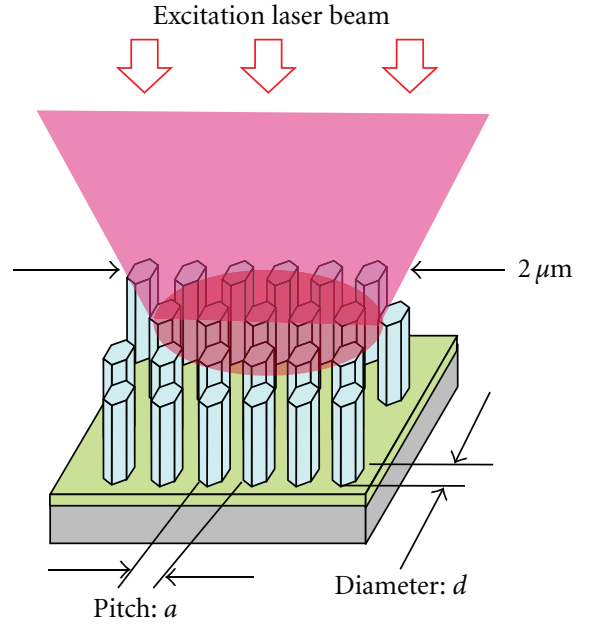

FIGURE 3: Schematic of substrate with nanowires irradiated by laser beam for PL measurements.

To confirm our speculation, we measured the $\mu$-PL for sample II at five different points in the same pattern (Figures $5(\mathrm{a})$ and 5(b)) and plotted the peak positions dependent on diameter in Figure 6. The thickness of the InGaAs layer was estimated to be about $100 \mathrm{~nm}$ or more for the nanopillars with various diameters and thus could be approximately considered as the bulk material. The indium content was determined by fitting the experimental data with the Varshni formula [38], and this was partly confirmed by the energy disperse X-ray (EDX) spectrum (about 9\%). We can clearly see that the indium content increases with an increase in the diameter of the nanopillars (Figure 6(c)). The indium content should be the same since the InGaAs layer had mainly grown on the top (111)B facet, if the ratio of the growth species containing $\mathrm{Ga}$ atoms to the growth species containing In atoms was the same for planar growth and for the growth on the top of the GaAs nanopillars. Consequently, the different indium content in the nanopillars with different diameters was due to the different diffusion coefficients between the growth species containing In atoms and the growth species containing Ga atoms on six (11-0) facets [3941]. The growth species containing $\mathrm{Ga}$ atoms had larger diffusion coefficients on six (1-10) facets than the growth species containing In atoms under our growth conditions. The difference between the peak positions from the InGaAs layer in samples II and I reflects the quantum confinement effect. Since the thickness of the InGaAs layer increases with a decrease in the diameter of the nanopillars, the quantum confinement effect decreases correspondingly. The fluctuations in the peak position are enhanced at the lower temperature, particularly for sample I, which is probably due to the fluctuations in the indium content within the nanopillars and the localization of electron-hole pairs associated with it. The full width at half maximum (FWHM) (around $20 \mathrm{meV}$ at $77 \mathrm{~K}$ ) of sample I is larger than that of the general InGaAs/GaAs QW [42]. The main reasons are given here. Since the excitation spot was about $2 \mu \mathrm{m}$ in diameter, the power density exposed onto the samples was estimated 


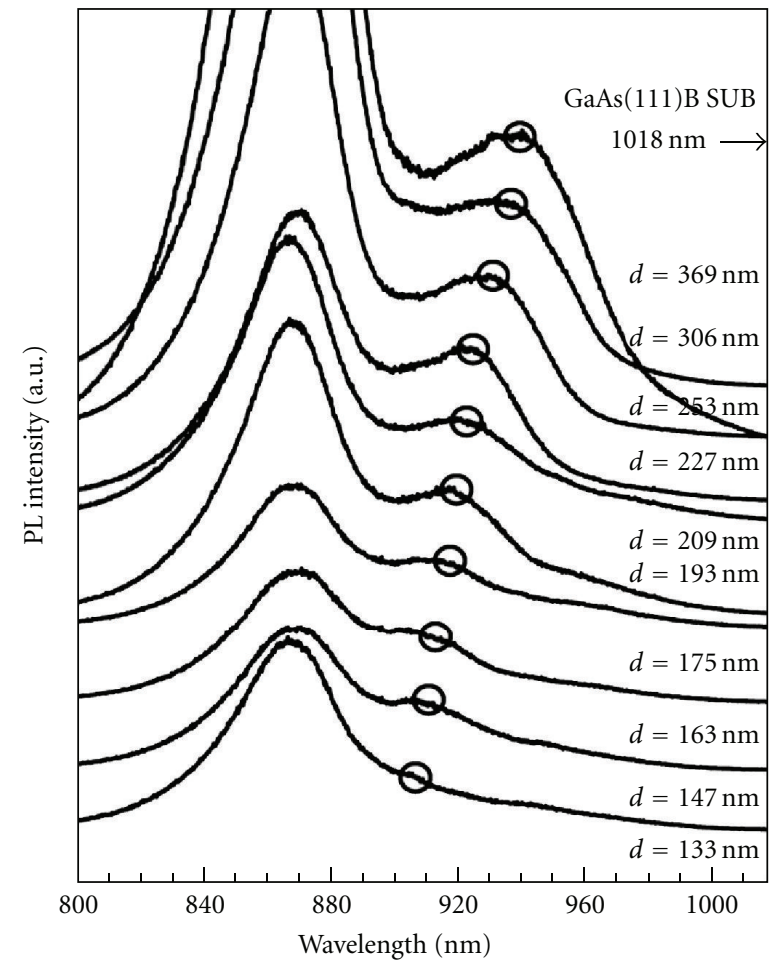

(a)

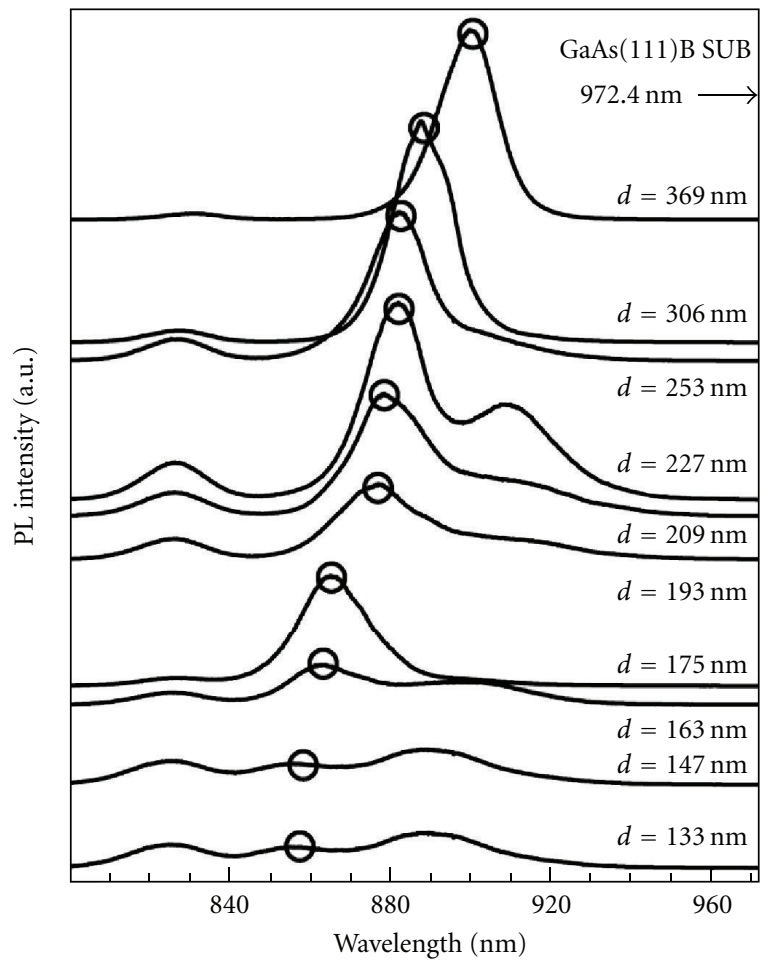

(b)

Figure 4: (a) Micro-PL spectra for sample I at room temperature (RT) and (b) at $77 \mathrm{~K}$ [28]. Open circles indicate the emission peaks of InGaAs.

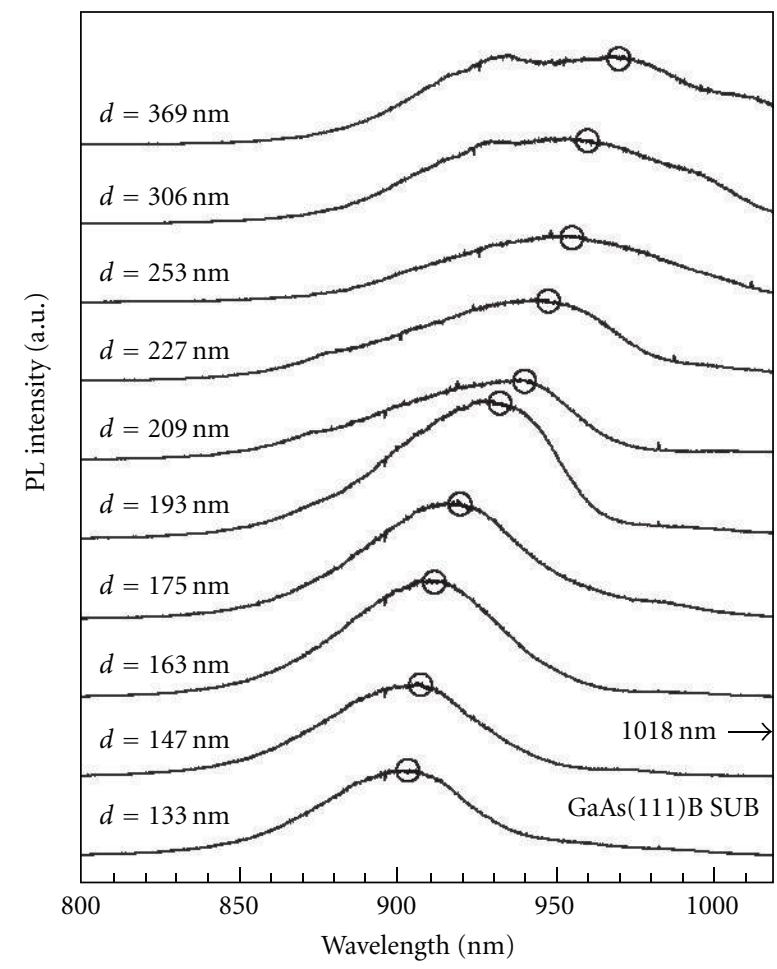

(a)

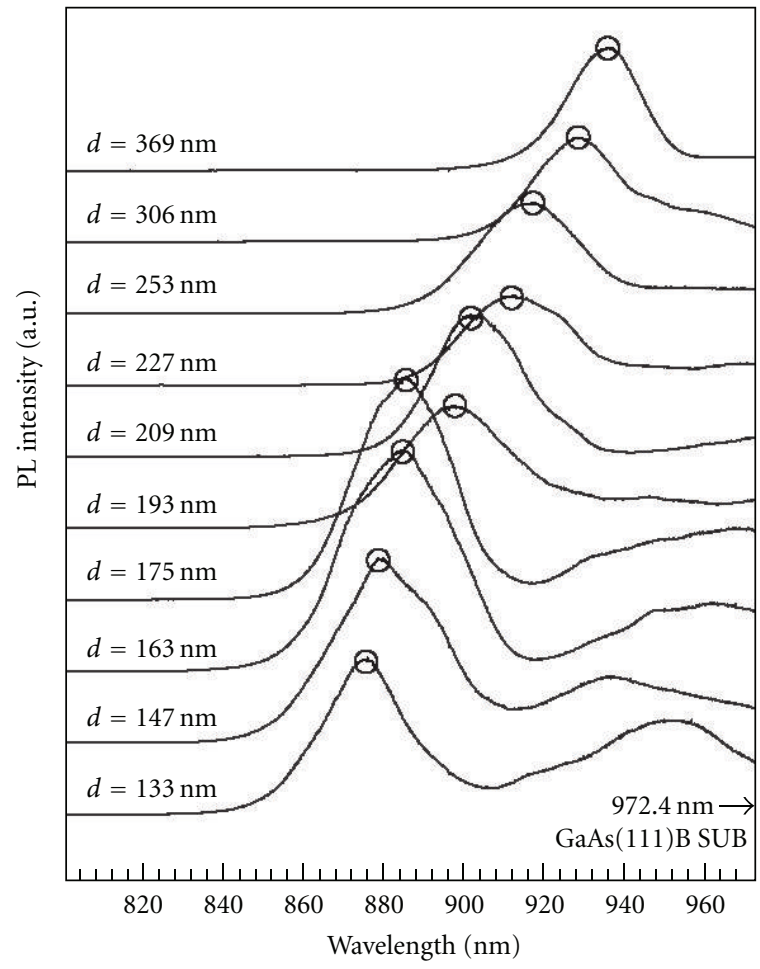

(b)

Figure 5: (a) Micro-PL spectra for sample II at RT and (b) at $77 \mathrm{~K}$ [28]. Open circles indicate the emission peaks of InGaAs. 


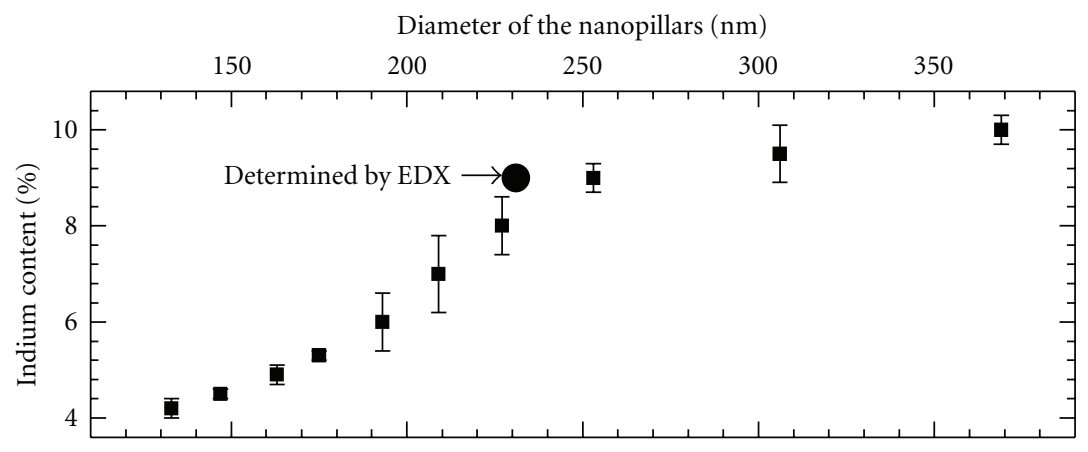

(c)

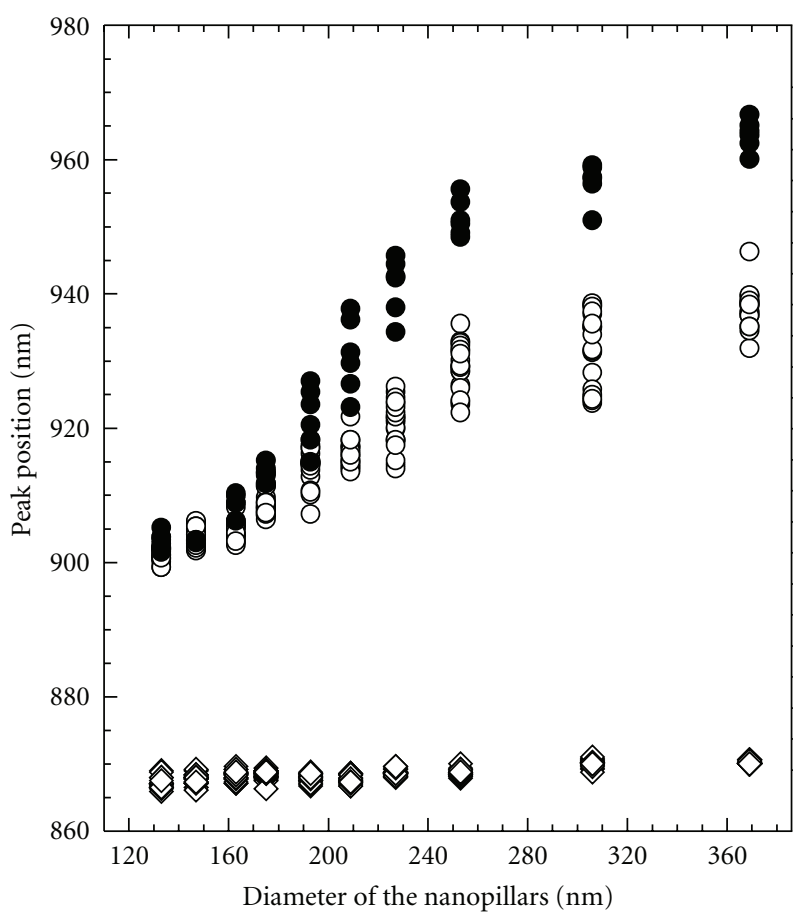

(a) At RT

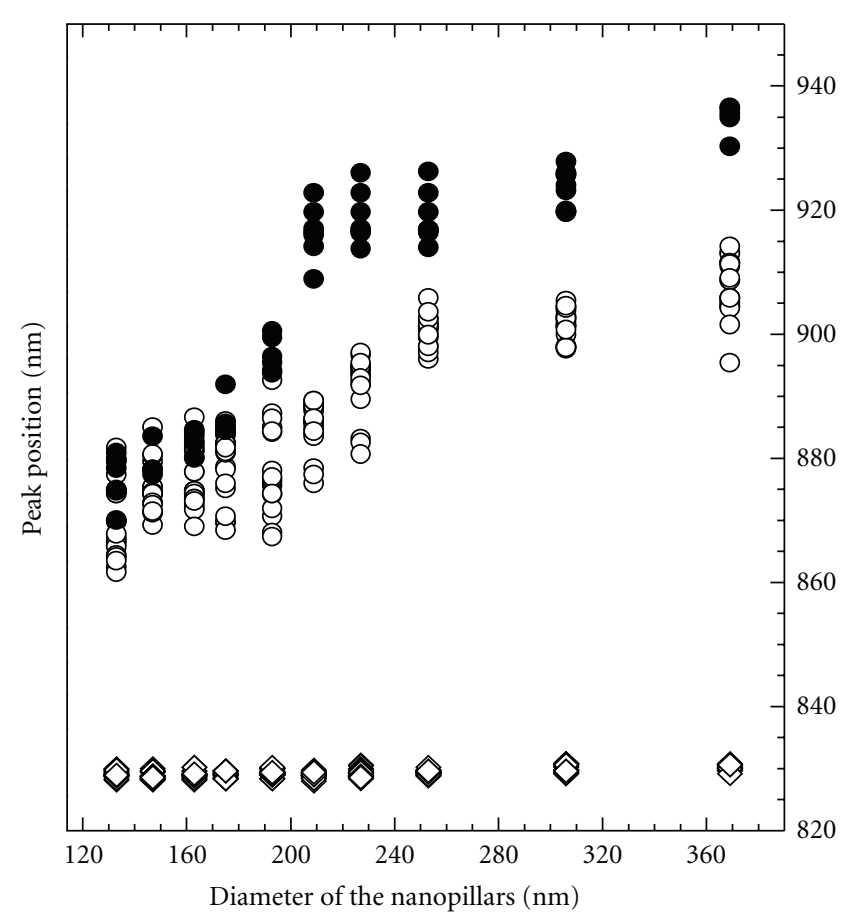

(b) At $77 \mathrm{~K}$

Figure 6: (a) Dependence of PL peak position on diameter of nanopillars at RT and (b) at $77 \mathrm{~K}$. Dependence of indium content on diameter of nanopillars (c). Open diamonds stand for band-edge emission of GaAs barrier layer in sample I, open circles for first electron heavy hole excitonic recombination of InGaAs/GaAs QW in sample I, and filled circles for band-edge emission of InGaAs layer in sample II [28].

to be $1 \mathrm{~kW} / \mathrm{cm}^{2}$ and more than ten nanopillars were excited simultaneously. Under such high power excitation conditions, some optical recombination occurred at $k \neq 0$ and the PL spectrum was broadened [42]. Due to the diameter and height fluctuations of the nanopillars, the thickness of the InGaAs well layer fluctuated slightly for nanopillars with different diameters, which also enhanced the FWHM of sample I. Another possible reason for this large FWHM is the fluctuations in the layer thickness at the interface of the InGaAs/GaAs QW.

A more quantitative analysis of the PL spectra with a piezoelectric effect due to the lattice mismatched (strained) layer structure has been reported [43], where the InGaAs QW thickness along the $\langle 111\rangle$ was estimated at $11.2 \mathrm{~nm}$.
3.2. GaAs/AlGaAs Vertical Structure with GaAs Quantum Well [30]. AlGaAs/GaAs vertical heterostructure NWs are attractive for fabricating a NW light-emitting device at a wavelength of $850 \mathrm{~nm}$. We could ignore the effect of lattice mismatching for the AlGaAs/GaAs heterojunction between GaAs and AlGaAs on the change in the bandgap energy of a GaAs QW compared with that of GaAs/InGaAs or GaAs/InGaP lattice-mismatched junctions. Therefore, we could control the emission wavelength of the GaAs QW by changing the size of the QW in the axial/lateral directions. To date, the number of reports on $\mathrm{AlGaAs} / \mathrm{GaAs}$ heterostructure NWs with a GaAs QW contained along the axial direction has been limited [44-47], due mainly to difficulty in controlling the size and the shape of AlGaAs. We also need to control 
and optimize the growth conditions to fabricate a QW or a quantum dot (QD) buried along the axial direction of NW.

The formation of an NW sample with a GaAs QW buried in AlGaAs began with GaAs core growth, followed by the growth sequence of an AlGaAs shell, a GaAs QW, an AlGaAs shell, and a GaAs cap layer. The growth temperature was $750^{\circ} \mathrm{C}$ for the GaAs core and QW layer, and the growth times were $5 \mathrm{~min}$ for the core and changed from 3 to $8 \mathrm{sec}$ for QWs, respectively. The partial pressure for $\mathrm{AsH}_{3}$ was $5.0 \times 10^{-4} \mathrm{~atm}$ and $2.7 \times 10^{-6}$ atm for TMG. Optimum growth conditions for the AlGaAs shell were chosen from preliminary experiments by changing the growth temperature between 800 and $850^{\circ} \mathrm{C}$ and the partial pressures of $\mathrm{AsH}_{3}$ between $2.6 \times 10^{-4}$ and $1.6 \times 10^{-3}$ atm, TMG between $9.2 \times 10^{-7}$ and $2.8 \times$ $10^{-6} \mathrm{~atm}$, and TMA between $1.6 \times 10^{-7}$ and $4.8 \times 10^{-7} \mathrm{~atm}$. The optimum growth temperature and the growth time for the AlGaAs shell layer were $800^{\circ} \mathrm{C}$ and $2.5 \mathrm{~min}$, where the partial pressures for $\mathrm{AsH}_{3}, \mathrm{TMG}$, and TMA corresponded to $7.8 \times 10^{-4}, 1.8 \times 10^{-6}$, and $3.2 \times 10^{-7}$ atm. After the GaAs QW layer had been grown, an AlGaAs layer was grown for $20 \mathrm{sec}$ at $750^{\circ} \mathrm{C}$ under the same source gas pressures as those for the AlGaAs shell-layer growth, where the growth temperature was changed beforehand from 750 to $800^{\circ} \mathrm{C}$ and the growth time was $2.5 \mathrm{~min}$.

To confirm whether the GaAs QW layer had grown on top of the (111)B facet or on the six $(-110)$ side facets of the AlGaAs shell, a GaAs layer was grown after the GaAs/AlGaAs core-shell NW had formed. Figures 7 (a) to 7 (c) are SEM images of GaAs NWs, GaAs/AlGaAs core-shell NWs (coreshell NWs) and GaAs/AlGaAs/GaAs heterostructure NWs (heterostructure NWs). Figure 7(d) shows the relationship between the height and diameter of NWs corresponding to the three growth steps in Figures 7 (a) to $7(\mathrm{c})$. Both the height and diameter of the NWs increased during the growth steps from the GaAs NW to the core-shell NW. From the comparison of the core-shell NW with the heterostructure NW, the heterostructure NW was higher by $300 \mathrm{~nm}$ on average than the core-shell NW, but the diameter increased by about $10 \mathrm{~nm}$, which indicates that the GaAs layer grew dominantly on the top surface of the GaAs/AlGaAs coreshell NW. We think that once a very thin GaAs layer had been deposited on the side $(-110)$ facets of AlGaAs, the thin GaAs layer masked the active AlAs surface and the surface diffusion length of Ga atoms increased to that of GaAs NW growth, as compared with the AlGaAs NW growth discussed in the previous section. Figures $7(\mathrm{e})$ to $7(\mathrm{~h})$ demonstrate how the core-shell structure effectively reduced the NW diameter compared with an AlGaAs/GaAs NW structure grown without the GaAs core. As can be seen in the SEM images in Figures $7(\mathrm{~g})$ and $7(\mathrm{~h})$, we reduced the diameter of the GaAs top layer, by about $190 \mathrm{~nm}$, to $150 \mathrm{~nm}$. We could estimate the thickness of a GaAs layer deposited on the side facet of the GaAs/AlGaAs core-shell NW to be less than $0.3 \mathrm{~nm}$ from these results when a GaAs QW as thin as 10$20 \mathrm{~nm}$ was grown on top of AlGaAs.

Figure 8(a) shows SEM images of heterostructure NWs with a GaAs QW buried in AlGaAs shells and a schematic cross-sectional structure (on the left). The height of the NWs was about $1.2 \mu \mathrm{m}$, and their diameters were $180-260 \mathrm{~nm}$. The diameter of the GaAs QW was assumed to be $140 \mathrm{~nm}$ for the structure, which was estimated from the change in NW diameters from the growth step of the core-shell NW to the heterostructure NW, as shown in the SEM images and the plot of the NW diameter in Figures 7(b), 7(c) and $7(\mathrm{~d})$. We think that the dispersion of the diameter for the core-shell NW (about $\pm 15 \mathrm{~nm}( \pm 11 \%)$ ) should be projected onto that of the GaAs QW. The diameter of the AlGaAs outer shell was designed to be about $180 \mathrm{~nm}$ ( $a$ target value) around the GaAs QW portion. Figure 8(b) shows the diameter distribution of the heterostructure NWs in Figure 8 (a). We found that standard deviation of diameter $\sigma_{\text {diameter }}$ of the NWs was about $29 \mathrm{~nm}$ and the uniformity of the diameter calculated using the ratio of $\sigma_{\text {diameter }} / d_{\text {ave }}$ ( $d_{\text {ave }}$ : average diameter) was $14 \%$. The apparent asymmetry in the histogram of the NW diameter in Figure 8(b) might be specific to the GaAs/AlGaAs heterostructure NWs containing a GaAs QW. When we grew AlGaAs NWs at $800^{\circ} \mathrm{C}$, the histogram of the diameter indicated a symmetric distribution with $\sigma_{\text {diameter }}$ of $21 \mathrm{~nm}$. Motohisa et al. [20] reported that the histograms for the diameter of GaAs NWs indicated a symmetric distribution with $\sigma_{\text {diameter }}=7-9 \mathrm{~nm}$ but the degree of symmetry was slightly affected by the proximity effect of electron-beam lithography when the pitch of the $\mathrm{SiO}_{2}$ mask openings was reduced from 0.6 to $0.4 \mu \mathrm{m}$. The standard deviation $(\sigma)$ of NW diameters for the AlGaAs NWs or the GaAs/AlGaAs heterostructure NWs with a GaAs QW was roughly 2-4 times larger than that for the GaAs NWs. We think the increase in diameter dispersion might be attributed to AlGaAs growth.

We measured the $\mu$-PL of the heterostructure NWs at $4.2 \mathrm{~K}$. A He-Ne laser (wavelength: $\lambda=632.8 \mathrm{~nm}$ ) was used in this experiment as an excitation source with a maximum excitation power density of about $1 \mathrm{~kW} / \mathrm{cm}^{2}$ and was focused onto about a $2-\mu \mathrm{m}$ diameter on the sample surface. Figure 9 shows the PL spectra for the three types of heterostructure NWs with a GaAs QW grown for growth times of 3, 5, and $8 \mathrm{sec}$. A PL spectrum of GaAs NWs has also been plotted as a reference. The pitch of the NWs plotted in Figure 9 was $1 \mu \mathrm{m}$. So the number of NWs excited by the laser beam was 3-5. The PL spectra for the three types of heterostructure NWs exhibit strong emission peaks near a photon energy of $1.5 \mathrm{eV}$, which might have originated from carbon acceptorrelated recombination emissions. The PL spectrum of GaAs NWs also displays a main peak near $1.49-1.50 \mathrm{eV}$, which is about $20 \mathrm{meV}$ below the second main peak at $1.515 \mathrm{eV}$, corresponding to band-to-band (band edge) recombination emissions. We can see a weak peak at $1.546 \mathrm{eV}$ for the heterostructure NW with a GaAs QW grown at $8 \mathrm{sec}$. The peak energy shifted to $1.560 \mathrm{eV}$ for the sample with a GaAs QW grown at $3 \mathrm{sec}$. Noborisaka et al. [29] reported that the PL intensity of GaAs NWs was an order of magnitude weaker than that of GaAs/AlGaAs core-shell NWs. Our results coincided well with their measurements, and we confirmed that the AlGaAs shell worked as a passivation site for the GaAs core NW. The PL intensity of the GaAs 


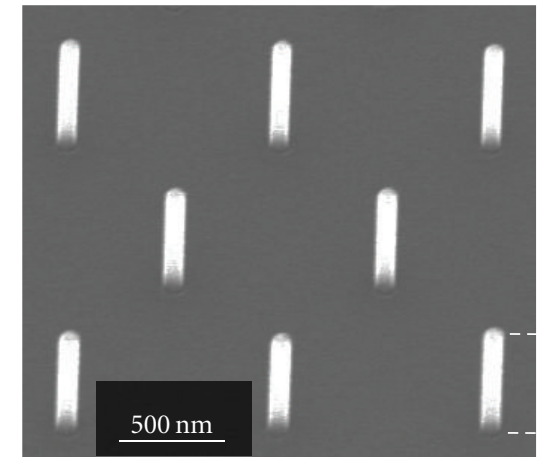

$d_{\mathrm{ave}}=90 \mathrm{~nm}$

(a) GaAs NW

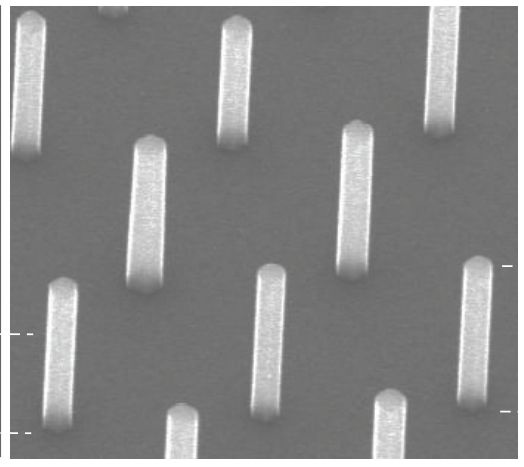

$d_{\mathrm{ave}}=140 \mathrm{~nm}$

(b) Core-shell NW

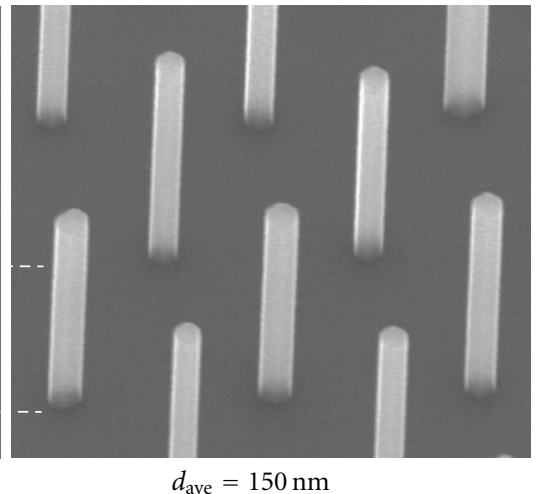

(c) Heterostructure NW

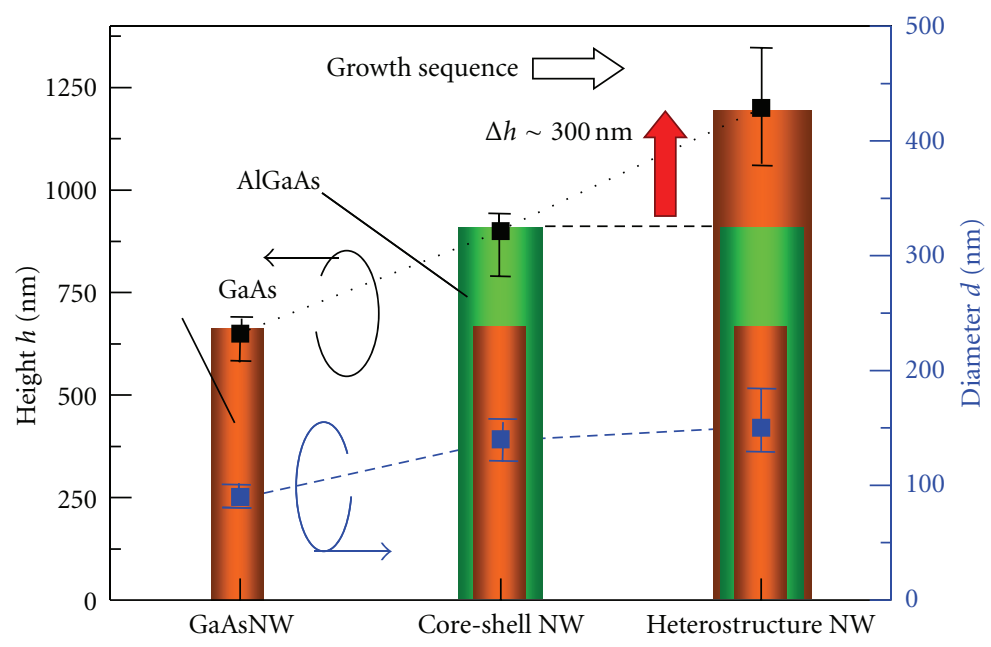

(d)

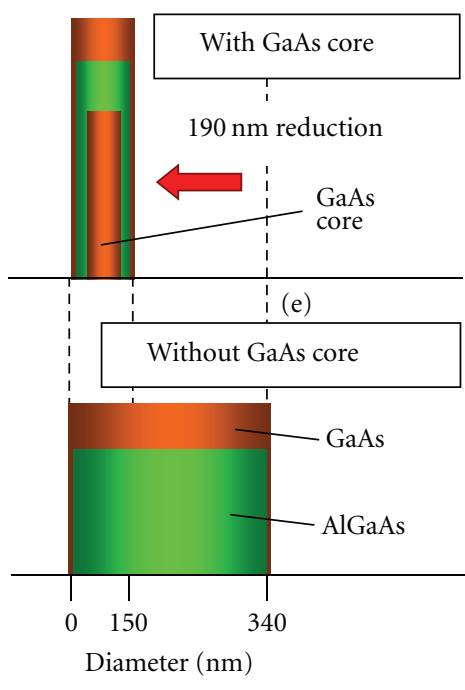

(f)

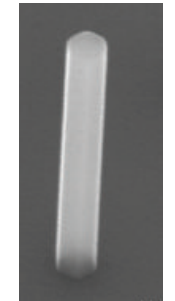

(g)

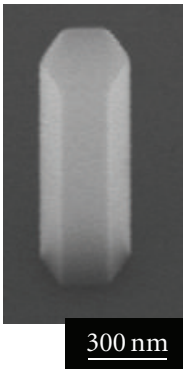

(h)

Figure 7: SEM images of NWs and changes in NW height and diameter. NW pitch $a=1.0 \mu \mathrm{m}$ and $\mathrm{SiO}_{2}$ mask opening size $d_{0}=80$ nm. (a), (b), and (c) correspond to SEM images for GaAs NWs, core-shell NWs, and heterostructure NWs. White dotted parallel lines bridged between SEM images (a) and (b), or (b) and (c) clarify how NW height changed between growth steps. (d) Changes in height and diameter of NWs corresponding to growth steps of GaAs NW (GaAs core growth time: 5 min), GaAs/AlGaAs core-shell NW (AlGaAs shell growth time: $2.5 \mathrm{~min}$ ), and GaAs/AlGaAs/GaAs heterostructure NW (top GaAs growth time: $2 \mathrm{~min}$ ). (e) and (f) illustrate reduced NW diameter by introducing GaAs core. (g) and (h) are SEM images of NW grown with and without GaAs core, corresponding to schematics in (e) and (f). Growth time for AlGaAs is $20 \mathrm{~min}$ and $5 \mathrm{~min}$ for GaAs for structures in (f) and (h) [30].

QW was one order of magnitude weaker than that of the acceptor-related emissions shown in Figure 9. We believe this was caused by two factors. The first was that the volume of the GaAs QW was about 1/30 of that of the GaAs core. The second factor was that the $\mathrm{Al}$ composition of the $\mathrm{AlGaAs}$ NW measured using PL measurements (not shown here) was about 0.11 , which was roughly equal to that of the outer shell measured using EDX. The difference in the energy bandgap between the GaAs QW and the AlGaAs shell was thus estimated to be as low as $0.11 \mathrm{eV}$, which is not sufficient to confine photoexcited carriers in the conduction band of the GaAs QW, compared with a GaAs QW sandwiched by AlAs barriers $[48,49]$. The relatively broad spectral feature of the GaAs QWs, when compared with that of the acceptorrelated emission peaks at $1.50 \mathrm{eV}$, might be originated from the dispersion in the GaAs QW thickness. We think this was caused by the dispersion in the NW height (Figure 8(a)).

We measured PL for three different areas on the sample surface for each growth time of the GaAs QW. Figure 10(a) plots the GaAs QW width (thickness along the $\langle 111\rangle$ direction) estimated from the PL peak energy versus that calculated by the GaAs NW growth rate along the $\langle 111\rangle$ direction. The inset in Figure 10(a) shows a dark-field scanning TEM (STEM) image of the top region of a heterostructure NW with a GaAs QW for a growth time of $5 \mathrm{sec}$. The depth profile for $\mathrm{Al}$ composition measured using EDX is plotted on the left side of the STEM image in Figure 10(a). The Al composition profile indicates different peak values above or below the GaAs QW. This could be attributed to different growth temperatures for the AlGaAs shells, but the reason 


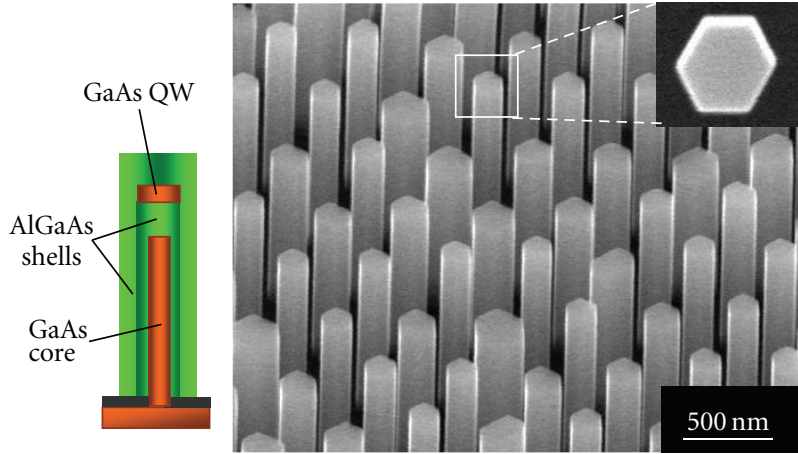

(a)

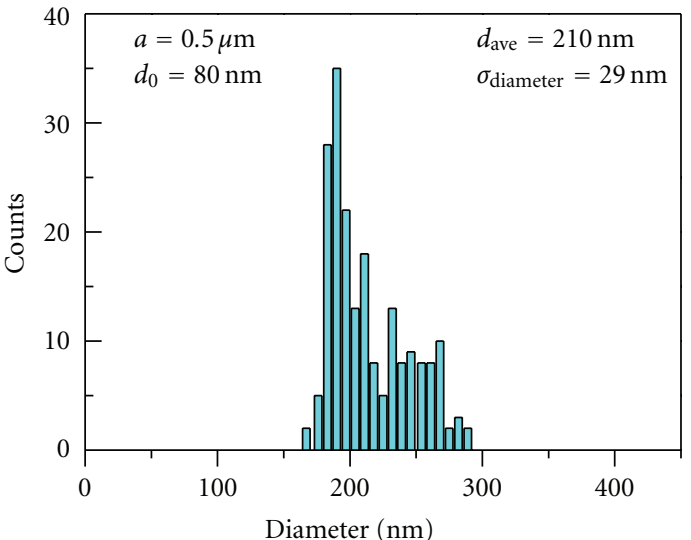

(b)

FIGURE 8: SEM images and schematic of structure of heterostructure NWs and distribution of diameters. (a) SEM images of grown NWs and schematic of cross-sectional structure of NW containing GaAs QW. GaAs cap layer is not shown in schematic. Inset SEM image in upper right is top view of NW. (b) Histogram of diameter distribution for NWs with pitch of $a=0.5 \mu \mathrm{m}$ [30].

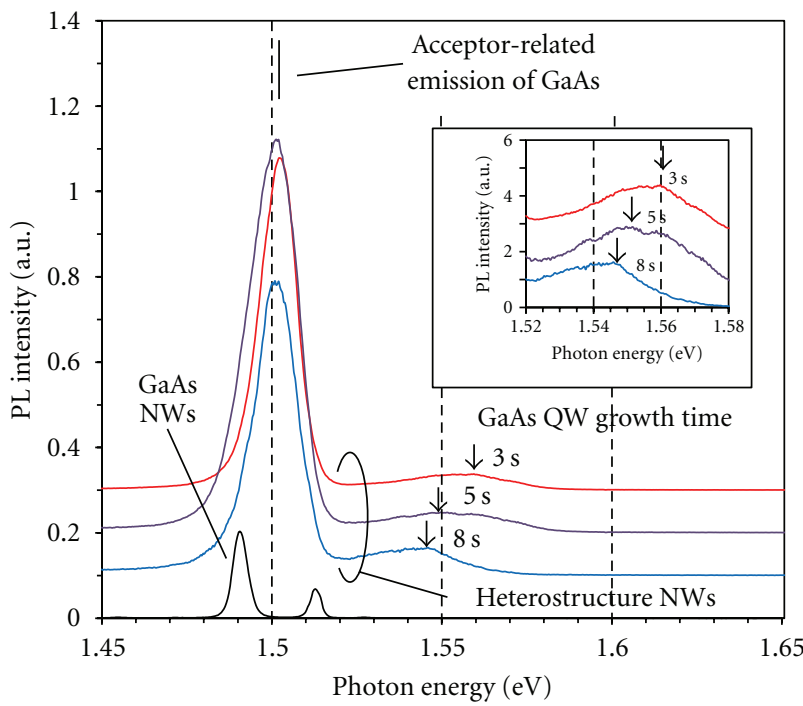

FIGURE 9: PL spectra for three types of heterostructure NWs with a GaAs QW grown for 3, 5, and $8 \mathrm{sec}$ and GaAs NWs (as a reference) measured at $4.2 \mathrm{~K}$. NW pitch was $1 \mu \mathrm{m}$ for all the NW samples. Inset is enlarged spectra for the GaAs QWs [30].

is not entirely clear. Figures $10(\mathrm{~b})$ and 10 (c) show a brightfield STEM image and a transmission electron diffraction pattern for the heterostructure NW, the analyzed part is corresponding to a segment from the GaAs QW region to the NW's top. There is a high-resolution TEM image of a center part of the NW top in Figure 10(d). There is a dark-field STEM image in Figure 10(e) corresponding to the top half of that in Figure 10(a). We found that the heterostructure NW with a GaAs QW had stacking faults perpendicular to the $\langle 111\rangle$ direction, which are distributed along the $\langle 111\rangle$ with a frequency ranging from a few nanometers to $20 \mathrm{~nm}$ as can be seen in Figure 10(b). The crystal structure was found to be zincblende with rotational twins around the $\langle 111\rangle$ axis (Figure 10(c)). The stacking faults originated from the rotational twins, which was confirmed by the change in the alignment of lattice image, and the positions are indicated by the arrows on the high-resolution TEM image taken near the top surface of the NW in Figure 10(d). In Figure 10(e), the positions for the GaAs QW and the AlGaAs barriers are indicated by the broken arrowed lines, which are corresponding to the areas of light-and-dark contrast on the STEM image except for the fine ones caused by stacking faults. We found that some of the stacking faults were distributed in the GaAs QW region from the STEM images in Figures 10(b) and 10(e). We think that the stacking faults developed at some stages of layer growth along the $\langle 111\rangle \mathrm{B}$ direction. But it is not clear whether they propagated from the GaAs core to the AlGaAs shell.

We used formulas for the optical transition between the energy levels of electrons and holes to obtain the QW width from the PL measurements, which are given below $[49,50]$ :

$$
\hbar \omega=E_{g}(\mathrm{GaAs})+E_{1}+E_{\mathrm{hh} 1},
$$

and quantized energy level $E$ for electrons or holes with effective mass $m$ is given by

$$
E=\left(\frac{\hbar^{2} \pi^{2}}{2 m}\right)\left(\frac{n}{L_{\mathrm{QW}}}\right)^{2},
$$

where $\hbar=h / 2 \pi$ ( $h$ : Planck's constant), $\omega$ is the angular frequency of light, and $E_{g}(\mathrm{GaAs})$ is the GaAs bandgap. Here, $n$ is a positive integer, $E_{1}$ is the lowest energy level of electrons in the conduction band, $E_{\mathrm{hh} 1}$ is the lowest energy level of heavy holes in the valence band, and $L_{\mathrm{QW}}$ is the width of the GaAs QW along the axial direction of the NW. The effective masses for the electrons and heavy holes used for fitting were $m_{e}^{*}=0.067$ and $m_{\mathrm{hh}}^{*}=0.45[49,50]$. For the strict treatment of the PL peak energy analysis of the GaAs QWs, we need to consider the finite energy barriers of AlGaAs, but here we assumed that the energy barrier height was infinite 


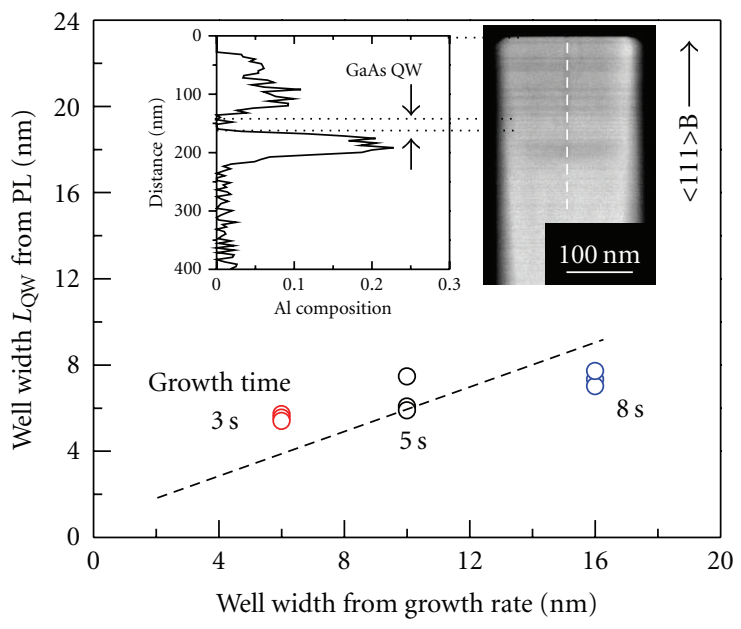

(a)

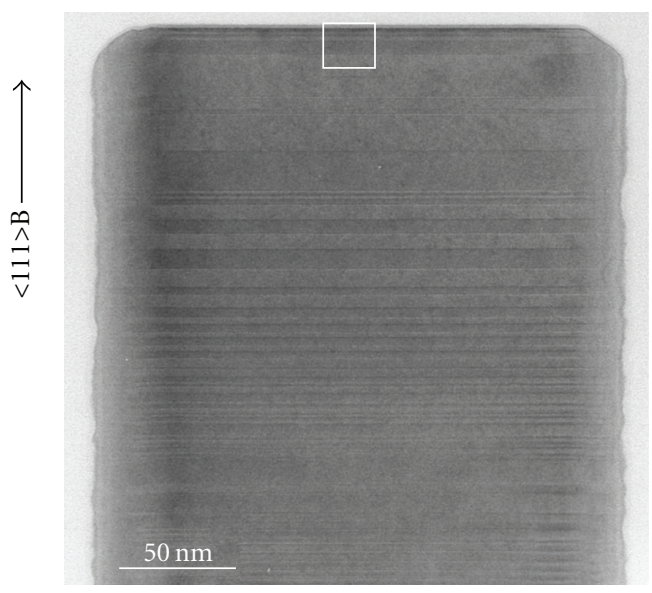

(b)

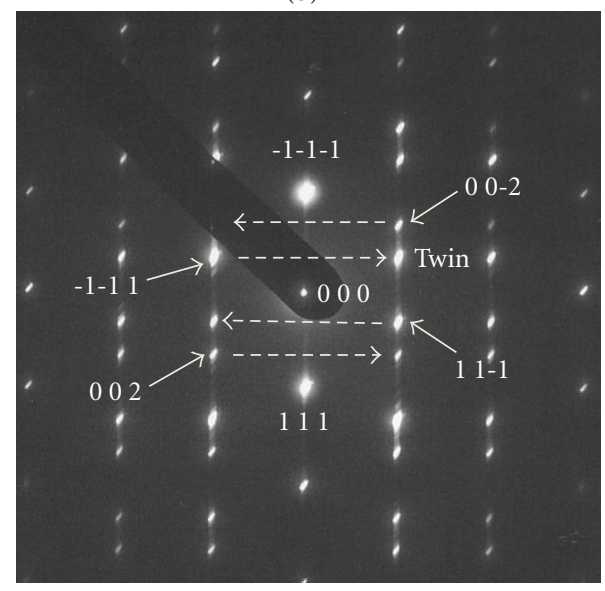

(c)

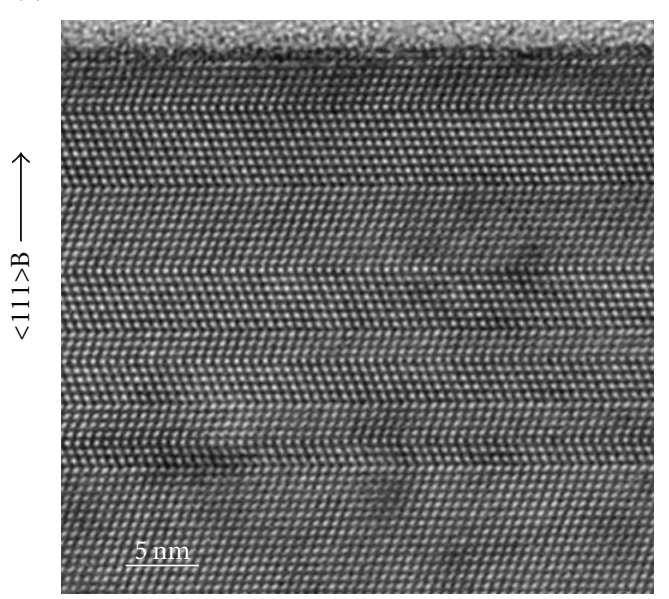

Top surface

(d)

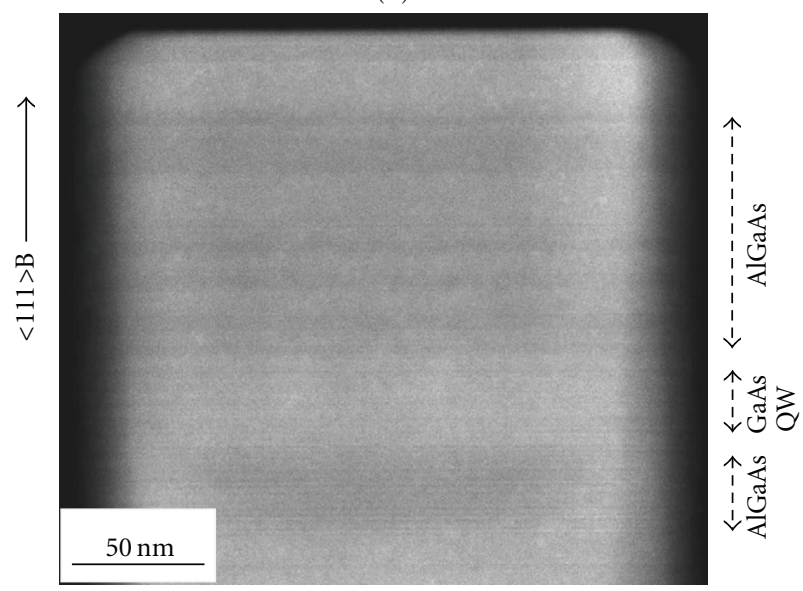

(e)

FIGURE 10: GaAs QW width calculated from PL spectra and growth rate and analysis by TEM. (a) Comparison of GaAs QW width obtained from PL peak energy with that estimated from axial growth rate of GaAs NW. NW pitch was $1 \mu \mathrm{m}$. Inset in upper right is dark-field STEM image of heterostructure NW taken with electron-beam direction parallel to $\langle-110\rangle$. Inset at left above has depth profile of Al composition measured from top of NW by using EDX, which indicates GaAs QW portion sandwiched by AlGaAs. White broken line along 〈111〉 direction on STEM image indicates path scanned by electron beam for EDX. (b) Bright-field STEM image of NW. (c) Transmission electron diffraction pattern. (d) High-resolution TEM image of NW top portion indicated by white rectangle on STEM image of (b). Twin boundaries are indicated by arrows. (e) Dark-field STEM image of (b). Layer positions of GaAs QW and AlGaAs barriers estimated along 〈111〉 direction are indicated by broken arrows [30]. 
and quantum confinement was only in the $\langle 111\rangle$ direction and ignored lateral confinement perpendicular to the $\langle 111\rangle$ direction for simplicity. We find this simple approximation can give some difference in the QW thickness (Figure 10(a)). The depth profile of the $\mathrm{Al}$ composition shown in the inset in Figure 10(a) indicates that a GaAs QW with a thickness of 5-20 nm was formed about $150 \mathrm{~nm}$ from the top surface of the heterostructure NW. The position of the GaAs/AlGaAs heterojunction interface looks diffuse (showing ambiguity of $\pm 10 \mathrm{~nm}$ ) from the dark-field STEM image in Figure 10(e), but we could estimate the thickness of the GaAs QW along the $\langle 111\rangle$ was roughly $30 \mathrm{~nm}$, which is wider than that obtained by EDX. We could not find the GaAs/AlGaAs coreshell interface along the lateral direction of the GaAs QW in the dark-field STEM image, because we could hardly see any light-and-dark contrast laterally. Tambe et al. [51] discussed the heterojunction interface structure of a GaAs/ $\mathrm{Al}_{x} \mathrm{Ga}_{1-x} \mathrm{As}$ core-shell NW from the images obtained with dark-field STEM. They used an Al composition of $x=0.9$ for the sample, and they obtained high contrast at the interface. We think the difference in the GaAs QW thickness measured by EDX and that from the STEM image was caused by low Al content in the AlGaAs barriers compared with the one that Tambe et al. reported. The GaAs QW thickness measured using EDX is in fair agreement to a certain extent with that estimated with the PL spectra or the GaAs NW growth rate for the QW growth time of $5 \mathrm{sec}$. The results obtained by PL measurement combined with TEM/EDX analysis indicate successful fabrication of the GaAs QW using SA-MOVPE.

Figure 11 shows the PL spectra for heterostructure NWs with a GaAs QW grown with different NW pitches from 0.5 to $3.0 \mu \mathrm{m}$. The growth time for the GaAs QW was $5 \mathrm{sec}$. We found a broad weak peak for the GaAs QW at the $50-70 \mathrm{meV}$ higher energy side of a strong emission peak near $1.5 \mathrm{eV}$, corresponding to acceptor-related recombination emissions. The broad weak peak position shifted to a higher energy region from 1.548 to $1.567 \mathrm{eV}$ as the NW pitch increased from 0.5 to $3.0 \mu \mathrm{m}$. We found the PL intensity weakened as the NW pitch changed from 0.5 to $3 \mu \mathrm{m}$. This was caused by the decrease in the number of NWs excited by the laser beam as the pitch increased. The graph in the inset at the upper right of Figure 11 indicates that the GaAs QW width estimated from the PL peak energy decreased as the NW pitch increased. This means the GaAs growth rate along the $\langle 111\rangle \mathrm{B}$ direction decreased on top of AlGaAs as the NW pitch increased, which is further evidence of the dependence of the growth rate of GaAs NWs on the NW pitch discussed by Noborisaka et al. [34]. The broad distribution of GaAs QW thickness along the $\langle 111\rangle$ direction might be a cause of the broad PL peaks for the GaAs QW compared with the relatively sharp acceptor-related emission peaks of the GaAs core. We found that there was a distribution of NW heights, as can be seen from the SEM image in Figure 8(a). The change in NW heights implies a distribution of GaAs QW thicknesses buried inside, which means spectral broadening of PL.

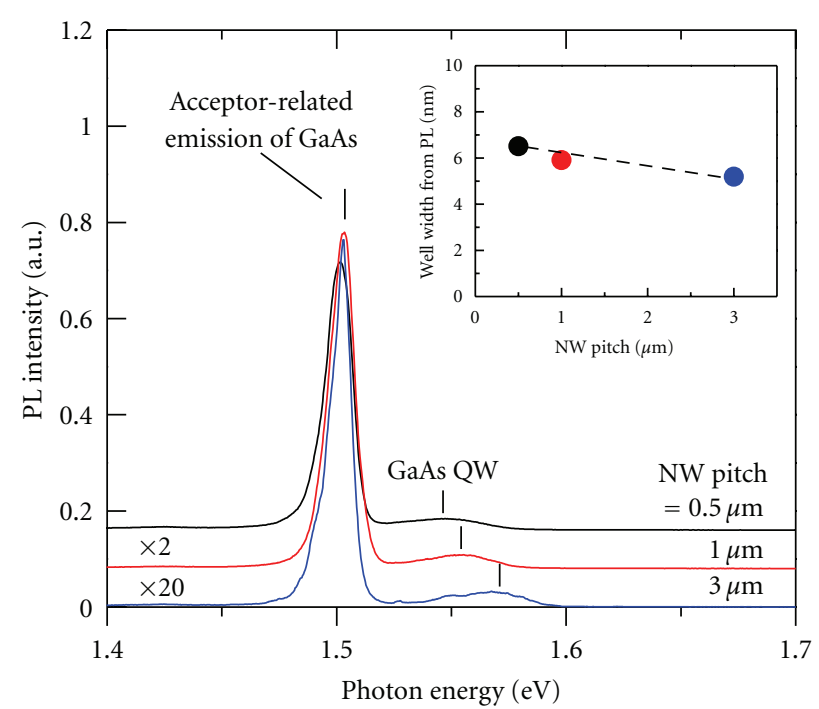

FIGURE 11: Dependence of PL spectra on NW pitch for heterostructure NWs containing GaAs QW. Graph in insert indicates GaAs QW width as function of NW pitch [30].

3.3. GaAs/GaAsP Vertical Structure with GaAs Quantum Well [33]. Another material we used as a barrier layer when fabricating a vertical heterostructure with a GaAs QW was GaAsP, which should be less affected by oxidation compared with materials containing Al like AlGaAs. Several reports have been published on GaAsP NW growth [52-55], but, to the best of our knowledge, only one [32] has been published on such growth using a catalyst-free method. Here we start with GaAsP NW growth then discuss GaAs/GaAsP heterostructure fabrication.

The source materials were TMG, tertiarybutylphosphine $\left(\left(\mathrm{C}_{4} \mathrm{H}_{9}\right) \mathrm{PH}_{2}\right.$ : TBP $)$, and $\mathrm{AsH}_{3}(20 \%$ in hydrogen $)$. The growth temperatures of GaAsP NWs ranged between 750 and $775^{\circ} \mathrm{C}$, and the growth time was $20 \mathrm{~min}$. The partial pressures of TMG were from $2.7 \times 10^{-6}$ to $4.1 \times 10^{-6} \mathrm{~atm}$, those of TBP were from $4.2 \times 10^{-5}$ to $2.5 \times 10^{-4} \mathrm{~atm}$, and those of $\mathrm{AsH}_{3}$ were from $4.2 \times 10^{-5}$ to $2.5 \times 10^{-4} \mathrm{~atm}$. The $\left(\mathrm{TBP}+\mathrm{AsH}_{3}\right) /(\mathrm{TMG})$ ratios (V/III ratios) were from 20 to 185. The optimum growth conditions for the GaAsP NWs were chosen from preliminary experiments on GaAsP NW growth. The optimum growth temperature and growth time for the GaAsP NWs were $750^{\circ} \mathrm{C}$ and $5 \mathrm{~min}$, where the partial pressures for TMG, TBP, and $\mathrm{AsH}_{3}$ were $4.1 \times 10^{-6}, 8.3 \times$ $10^{-5}$, and $8.3 \times 10^{-5} \mathrm{~atm}$, respectively. The V/III ratio was 20 .

Figure 12 shows SEM images of GaAsP NWs grown at a V/III ratio of 20. We can see that the NWs have hexagonal and triangular structures and crystal facets, which indicate the formation of $(-110)$ equivalent side facets and a top (111)B facet. The NW diameter was rather dispersed compared with that of GaAs NWs, as can be seen in Figure 12. This might be attributable to As- and P-contained NW growth, but the reason is not yet clear.

Figure 13 shows the PL spectrum of GaAsP NWs grown at the V/III ratio of 20. The PL spectrum of the GaAs substrate has also been plotted with the blue line as a 


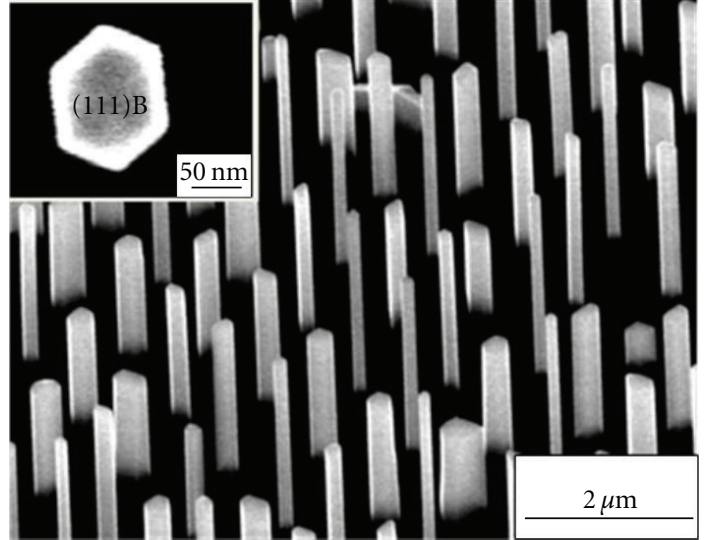

Figure 12: SEM images of GaAsP NWs grown at $750^{\circ} \mathrm{C}$ with V/III ratio of 20. Upper left inset is SEM image of top view of NW [33].

reference. The number of NWs excited by the laser beam was about 3-5 for the sample with a pitch of $1 \mu \mathrm{m}$. The PL spectrum of GaAsP NWs had a strong emission peak at a photon energy of $1.80 \mathrm{eV}$, which is clearly different from the main peak of the GaAs substrate. The full width at half maximum (FWHM) of the PL spectrum of the NWs was $18 \mathrm{meV}$. The PL peak energy and the FWHM did not change when we changed the position of laser excitation on the sample surface. This indicates that GaAsP NWs with an equal atomic composition were formed within the NW grown area. The FWHM values for the GaAsP NWs were as narrow as those for the InGaAs NWs [35]. We estimated the solid $P$ atomic content at $25 \%$ from the PL peak energy, that is, $\mathrm{GaAs}_{0.75} \mathrm{P}_{0.25}$ (single crystal GaAs $\mathrm{Gax}_{1-x} P_{x}$ layers had a direct bandgap between the valence band and the conduction band when solid $P$ atomic content $x$ was less than 0.44 [56]).

Here, we will explain the fabrication process for a GaAs QW buried in a heterostructure NW and the PL analysis to which it was subjected. To form a GaAs QW on top of a GaAsP NW and confine carriers in the QW threedimensionally, uniform hexagonal pillar structures with a reduced diameter should be formed. However, growing a GaAs QW directly onto the GaAsP NWs was a major challenge, because laterally growing GaAsP still created problems in forming a NW with a reduced diameter of less than $100 \mathrm{~nm}$ and good uniformity in shape for the NW array. To solve this, we selected a method of growing a GaAs NW (height: about $500 \mathrm{~nm}$, diameter: about $60 \mathrm{~nm}$ ) before we grew the first GaAsP NW shell and then grew a GaAs QW on top of the first GaAsP NW shell, which was similar to the procedure for growing the GaAs/AlGaAs vertical heterostructure with a GaAs QW [30]. We used a single growth temperature of $750^{\circ} \mathrm{C}$ and a V/III ratio of 20 to form the heterostructure based on the optimum growth conditions for GaAsP NWs.

Figures 14(a) and 14(b) show SEM images of heterostructure NWs with a GaAs QW buried in GaAsP and a schematic cross-sectional structure (on the right of Figure 14(b)). The pattern pitch and the mask-opening diameter we designed were $3 \mu \mathrm{m}$ and $60 \mathrm{~nm}$. The NW

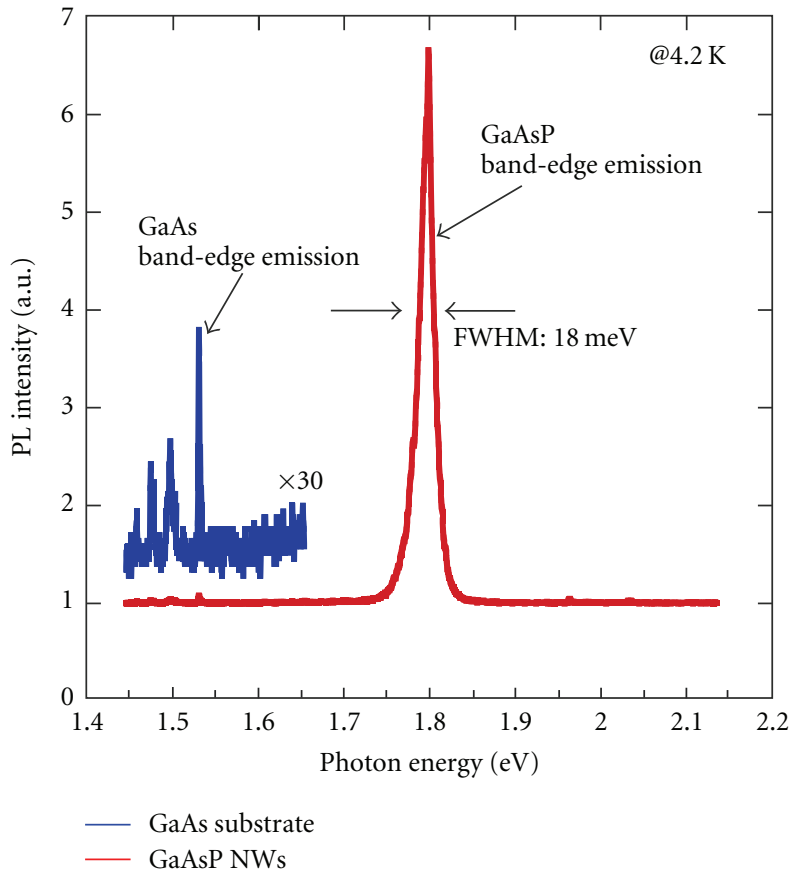

Figure 13: PL spectra for GaAsP NWs and GaAs substrate at $4.2 \mathrm{~K}$ [33].

height and diameter were approximately $630 \mathrm{~nm}$ and $80 \mathrm{~nm}$. The GaAs QW diameter was assumed to be $70 \mathrm{~nm}$ for the structure, which was estimated from the changes in diameter from GaAs (growth time: $t_{g}=5 \mathrm{~min}$ ) $/ \mathrm{GaAsP}$ $\left(t_{g}=5 \mathrm{~min}\right)$ single-heterostructure NWs to GaAs $\left(t_{g}=\right.$ $5 \mathrm{~min}) / \mathrm{GaAsP}\left(t_{g}=5 \mathrm{~min}\right) / \mathrm{GaAs}\left(t_{g}=5 \mathrm{~min}\right)$ doubleheterostructure NWs. By comparing the heterostructure NW (Figure 14) with the GaAsP NW (Figure 12), we can see that the uniformity in diameter was considerably improved by introducing GaAs NW growth before heterostructure growth.

We measured the $\mu$-PL of the heterostructure NW with a GaAs QW buried in GaAs/GaAsP NWs. Figures 15(a) and 15(b) show the PL spectra of the heterostructure NWs with a GaAs QW grown with different growth times $\left(t_{\mathrm{QW}}=1.5\right.$, 3.0 , and $4.5 \mathrm{sec})$ and different NW pitches $(0.5,1.0$, and $3.0 \mu \mathrm{m})$. A PL spectrum of a GaAs/GaAsP heterostructure NW (single heterostructure NW without QW) has been plotted in Figure 15(a) as a reference. The NW pitch is $3 \mu \mathrm{m}$, and one NW was excited by the laser beam for the spectra in Figure 15(a). The PL spectra for the four types of NWs in Figure 15(a) and for the three types of NWs in Figure 15(b) have strong emission peaks near a photon energy of $1.5 \mathrm{eV}$, which might have originated from the carbon acceptor-related recombination emissions in GaAs [57]. A short but clear peak for GaAs band-edge (bandto-band recombination) emissions can be confirmed at $1.515 \mathrm{eV}$, which is on the slightly higher energy side of the acceptor-related emission peak of GaAs. The peaks for GaAs band-edge emissions at $1.515 \mathrm{eV}$ were not observed for the GaAs/AlGaAs heterostructure NWs discussed in the previous section. We think this is a clear difference between 


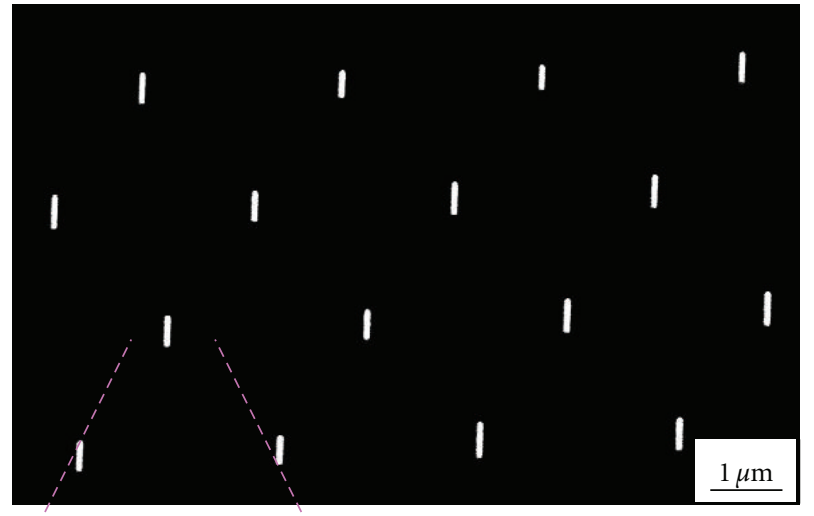

(a)

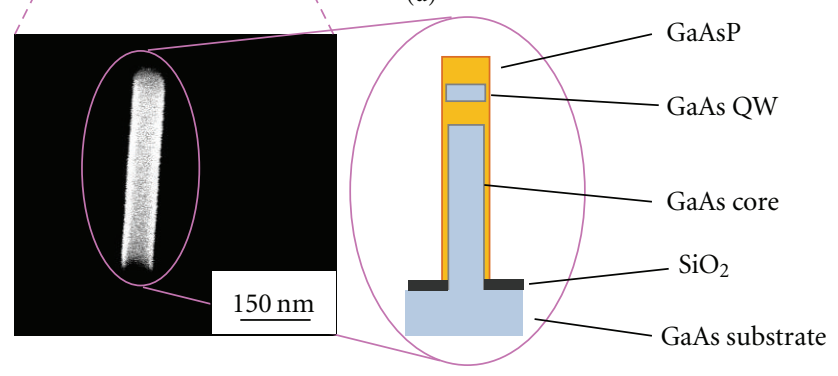

(b)

FIGURE 14: SEM images of heterostructure NWs with GaAs QW buried in GaAsP and schematic cross-sectional structure of NW containing GaAs QW. SEM image at lower left is enlarged view of NW [33].

the GaAs/GaAsP NWs and that GaAs/AlGaAs, whose quality might be affected by $\mathrm{Al}$ oxidation. The PL spectrum for the single heterostructure NW also indicates the main peak at $1.8 \mathrm{eV}$, which is almost the same as that of the GaAsP NWs with the pitch of $1 \mu \mathrm{m}$ (Figure 13). This indicates that the atomic content of the GaAsP shell was independent of the pitch. We can see clear emission peaks between 1.60 and $1.62 \mathrm{eV}$ for the heterostructure NWs with a GaAs QW, which is on the higher energy side of acceptor-related emissions and on the lower energy side of the GaAsP NW peak at $1.8 \mathrm{eV}$. The emission peak energy for the GaAs QW was increased as the time to grow the QW was decreased (Figure 15(a)) or the NW pitch was increased (Figure 15(b)). The GaAs QW thicknesses along the $\langle 111\rangle$ direction estimated by using the PL peak energy in Figures 15(a) and 15(b) and the approximation in the previous section (1) and (2) were $5.7-6.0 \mathrm{~nm}$, which were roughly equal to those grown sandwiched between AlGaAs. The number of NWs excited by the laser beam was about 10-12 for the sample with the pitch of $0.5 \mu \mathrm{m}$ and about 3-5 for the sample with the pitch of $1 \mu \mathrm{m}$. We estimated that the greater the number of NWs, the larger the dispersion of QW thicknesses. This is why the FWHM of the PL peak for the QW increased from 10 to $20 \mathrm{meV}$ as the pitch was decreased from 3.0 to $0.5 \mu \mathrm{m}$, as can be estimated from the PL spectral shapes in Figure 15(b). The relative PL intensity of the QW compared with that of the acceptor-related emission peak at $1.5 \mathrm{eV}$ for the GaAs/GaAsP structures (Figures 15(a) and 15(b)) is one order of magnitude greater than that for the GaAs/AlGaAs structures (Figures 9 and 11). Moreover, the FWHMs of the PL peaks for the QWs buried in GaAsP were smaller, by 30$50 \%$, than those buried in AlGaAs (Figures 9 and 11). This indicates that we can obtain GaAs QWs of better quality in GaAs/GaAsP NWs than in GaAs/AlGaAs NWs.

We carried out measurements with TEM/EDX to investigate the atomic content around a GaAs QW buried within GaAsP barrier layers. Figures $16(\mathrm{a})$ to $16(\mathrm{c})$ show darkfield STEM (DF-STEM) images and profiles of the atomic content of $\mathrm{Ga}$, As, and $\mathrm{P}$ measured along and across the $\langle 111\rangle$ direction. The DF-STEM image of the NW top portion is shown in Figure 16(b), in which a GaAs core and a GaAs QW are identified as bright portions compared with a relatively dark GaAsP portion. It should be observed that stacking faults (rotational twins around the $\langle 111\rangle$ axis) contained in the NW appear as segments with light-and-dark contrast in the STEM image. The atomic content profiles for Ga, As, and $\mathrm{P}$ detected by EDX scanned across the QW region (along the $\langle 111\rangle$ direction) and the NW trunk with the GaAs core are plotted on the right side of the STEM image in Figure 16(b). The profiles indicate that the QW, which is 10-nm thick, is located $70 \mathrm{~nm}$ below the NW's top, is separated $37 \mathrm{~nm}$ from the GaAs core, and is buried in $\mathrm{GaAs}_{0.80} \mathrm{P}_{0.20}$. The GaAs QW thickness measured from the EDX profile is roughly equal to that calculated from the GaAs growth rate along the $\langle 111\rangle$ direction and that obtained from the PL peak energy. We found that the GaAs core NW was not capped by a GaAsP layer within the resolution limit of EDX from the atomic content profile around the bottom half of the trunk shown in Figure 16(c).

\section{Heterostructure Growth in Radial Direction}

Radial (core-shell) heterostructure nanowires enable the passivation of interface states and thereby improve the overall performance of the semiconductor devices that result from these. Core-multishell nanowires formed by sequential modulation of the composition along the radial direction offer the distinct ability of enabling multifunctions to be incorporated into individual nanowires.

4.1. GaAs/AlGaAs Core-Shell Structure [29]. The formation of core-shell heterostructures by using lateral growth of AlGaAs shows an increase in the photoluminescence intensity in nanowires. We grew GaAs and AlGaAs in succession for core-shell heterostructures. The partial pressure of TMG for GaAs was $2.7 \times 10^{-6}$ atm and that for $\mathrm{AsH}_{3}$ was $5.0 \times$ $10^{-4} \mathrm{~atm}$. The partial pressures in the growth of the AlGaAs shell of the total group III precursors and $\mathrm{AsH}_{3}$ were the same as for GaAs growth. The growth temperature was $750^{\circ} \mathrm{C}$ for $\mathrm{GaAs}$ and $850^{\circ} \mathrm{C}$ for AlGaAs. Perfect selective epitaxy of both GaAs and AlGaAs was achieved under these conditions. The growth time was $20 \mathrm{~min}$ for GaAs and from 10 to $40 \mathrm{~min}$ for AlGaAs. Figure $17(a)$ is an SEM image of GaAs nanowires before AlGaAs was grown. A periodic array with a pitch of $1 \mu \mathrm{m}$ of free-standing GaAs nanowires with a height of $3 \mu \mathrm{m}$ was formed in the mask openings. The cross-section of the 


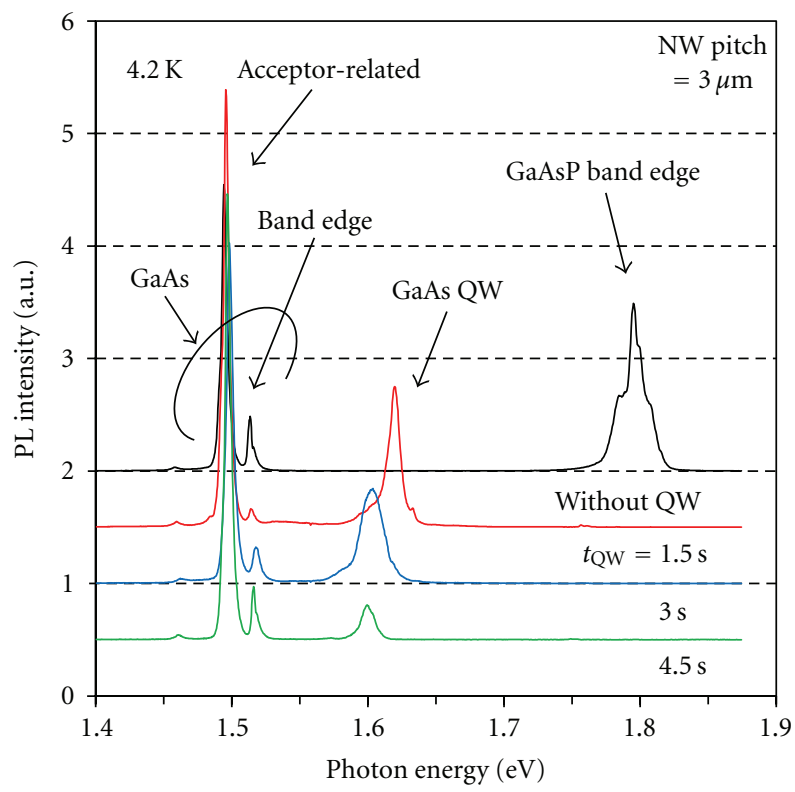

(a)

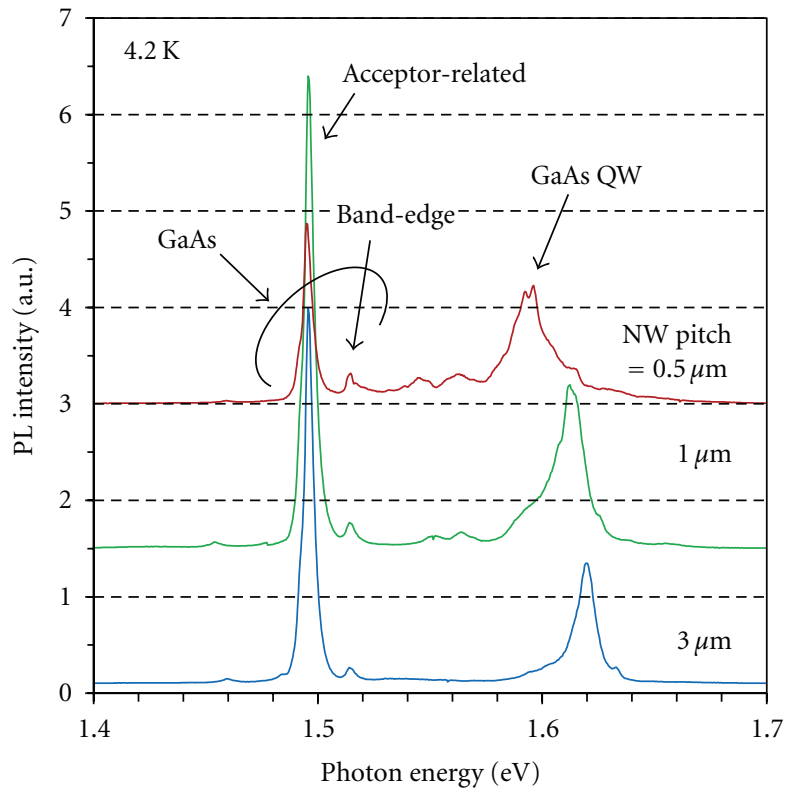

(b)

FIgURE 15: 4.2-K PL spectra for heterostructure NWs with GaAs QW buried in GaAsP. (a) PL spectra for NWs with GaAs QW for growth times $\left(t_{\mathrm{QW}}\right)$ of $1.5,3.0$, and $4.5 \mathrm{sec}$. NW pitch is $3 \mu \mathrm{m}$. PL spectrum for GaAsP/GaAs heterostructure NW without QW has been plotted as reference. (b) PL spectra for NWs with GaAs QW for NW pitches of $0.5,1.0$, and $3.0 \mu \mathrm{m}$. $t_{\mathrm{QW}}$ is $1.5 \mathrm{sec}$.

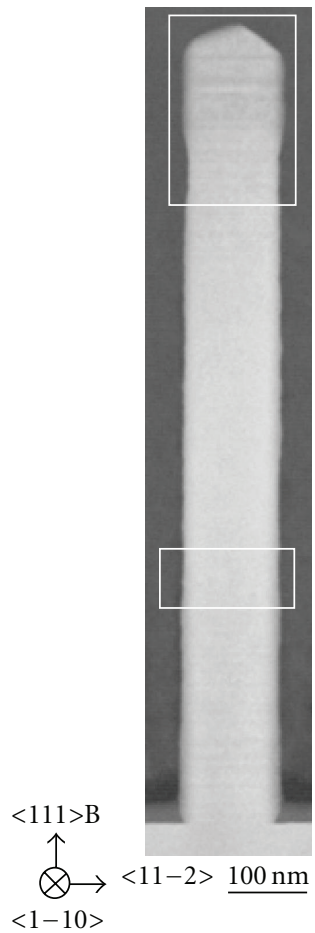

(a)
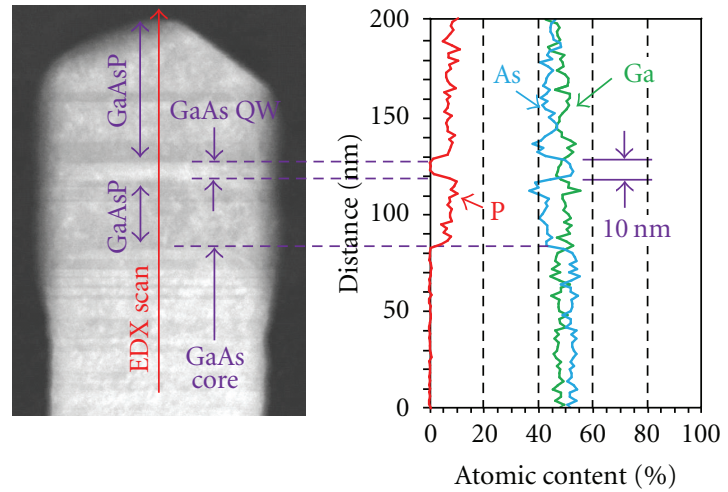

(b)

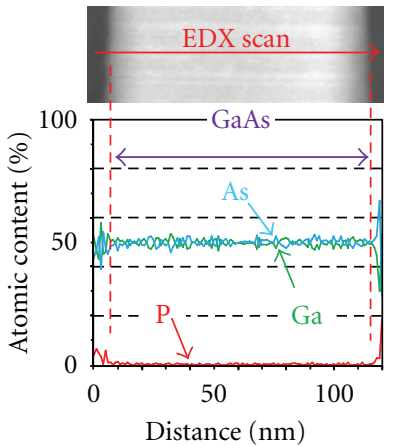

(c)

FIGURE 16: TEM/EDX analysis of heterostructure NW with GaAs QW. (a) Dark-field STEM (DF-STEM) image of NW. (b) DF-STEM image at top of NW of (a) and atomic content profiles for Ga, As, and P measured by EDX. (c) DF-STEM image at center-bottom part of NW and atomic content profiles obtained by EDX. 


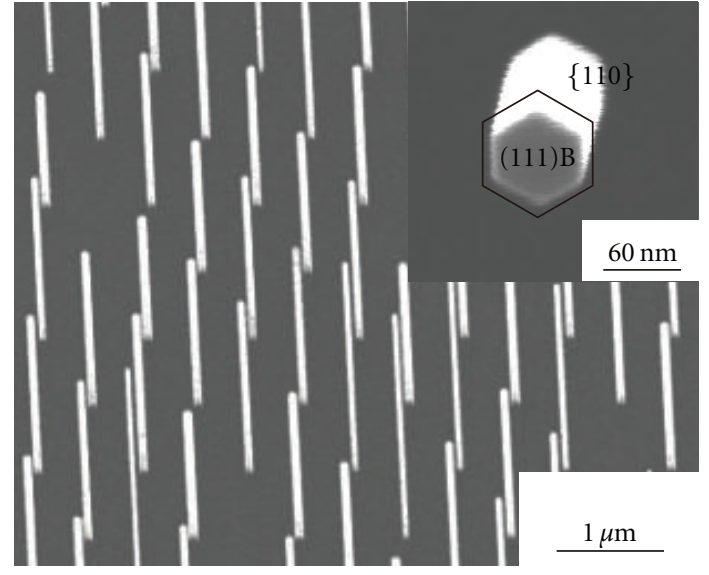

(a)

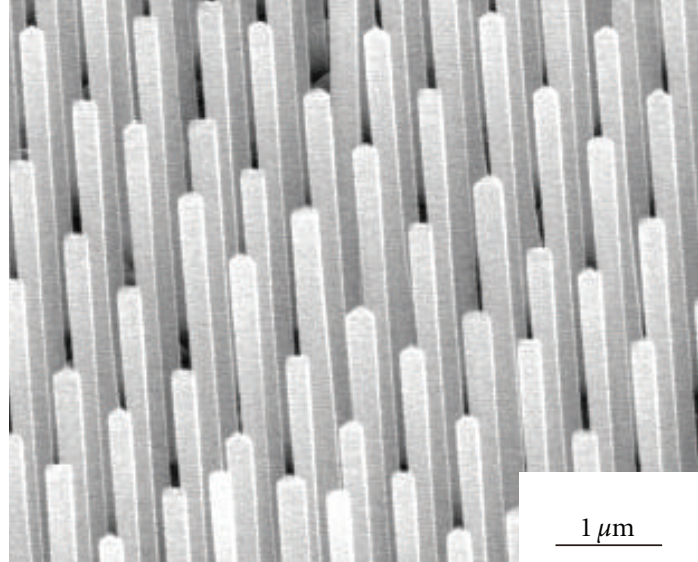

(b)

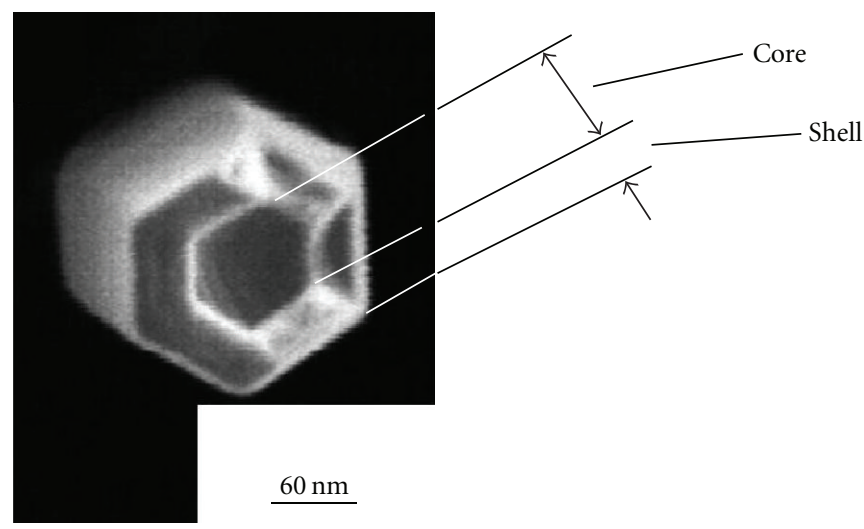

(c)

FIGURE 17: SEM images of grown nanowires. (a) SEM images of GaAs nanowires. Inset has image of nanowire from top. (b) SEM image of nanowires after growth of AlGaAs. (c) Top-view SEM image of GaAs/AlGaAs core-shell nanowire after preferential etching [29].

nanowires is hexagonal with well-defined sidewalls, as can be seen from the inset. Their diameters $d$ are from 50 to $100 \mathrm{~nm}$. Nonuniformity in their size originated mainly from the irregular mask-opening sizes. Figure 17(b) shows an SEM image of AlGaAs growth on GaAs nanowires. The growth time of AlGaAs was $10 \mathrm{~min}$. Although they maintained their hexagonal shapes, we found that the nanowires became taller $(5 \mu \mathrm{m})$ and thicker $(200-300 \mathrm{~nm})$.

Figure 17(c) has a top-view SEM image of a GaAs/ AlGaAs core-shell nanowire after a two-step etching process; specifically, anisotropic dry etching followed by preferential wet etching on core-shell nanowires. We used reactive ionbeam etching for dry etching with $\mathrm{CH}_{4}, \mathrm{H}_{2}, \mathrm{Ar}$, and $\mathrm{N}_{2}$ for 30 min and used a mixture of $\mathrm{NH}_{4} \mathrm{OH}$ and $\mathrm{H}_{2} \mathrm{O}_{2}$ for wet etching. We can clearly distinguish the GaAs core part with a diameter of $100 \mathrm{~nm}$ from the AlGaAs shell with an outer diameter of $200 \mathrm{~nm}$ from Figure 17(c). Here, the AlGaAs shell remained as a nanotube after the etching process. Figures 17(a) to 17(c) also indicate that AlGaAs was grown laterally on the sidewalls of GaAs nanowires as well as on their tops.

Lateral growth was confirmed by the dependence of nanowire diameter on the AlGaAs growth time shown in
Figure 18. We concluded from these results that free-standing GaAs/AlGaAs core-shell nanowires had been formed by using SA-MOVPE. In Figures 17(a)-17(c), the thickness of the AlGaAs shells is $75-100 \mathrm{~nm}$, and the top of the nanowires is capped with $2-\mu \mathrm{m}$-long AlGaAs. If we subtract the length of the capped AlGaAs on the top, the aspect ratio of the coreshell part of the nanowires is about 10 . As has previously been reported [37], the sidewalls of GaAs hexagonal nanowires are (110) facet surfaces, vertical to the (111) plane. The appearance of these facet sidewalls is because their growth rate is slower than that of (111)B. In addition, we found that diameter $d$ of the nanowires is equal to mask-opening diameter $d_{0}$ [37]. This means that the growth on (110) sidewalls is negligible for GaAs grown under the present conditions. Growth, on the other hand, takes place on the sidewall surfaces laterally as well as on the top surfaces for AlGaAs. Ando et al. have reported enhanced lateral growth of AlGaAs on the (110) sidewalls of selectively grown GaAs wire structures on (111)B under slightly different conditions [58]. The enhanced lateral growth on the sidewall (110) facet in AlGaAs could be explained by a stronger bonding and a shorter migration length of $\mathrm{Al}$ atoms on (110) surfaces. 


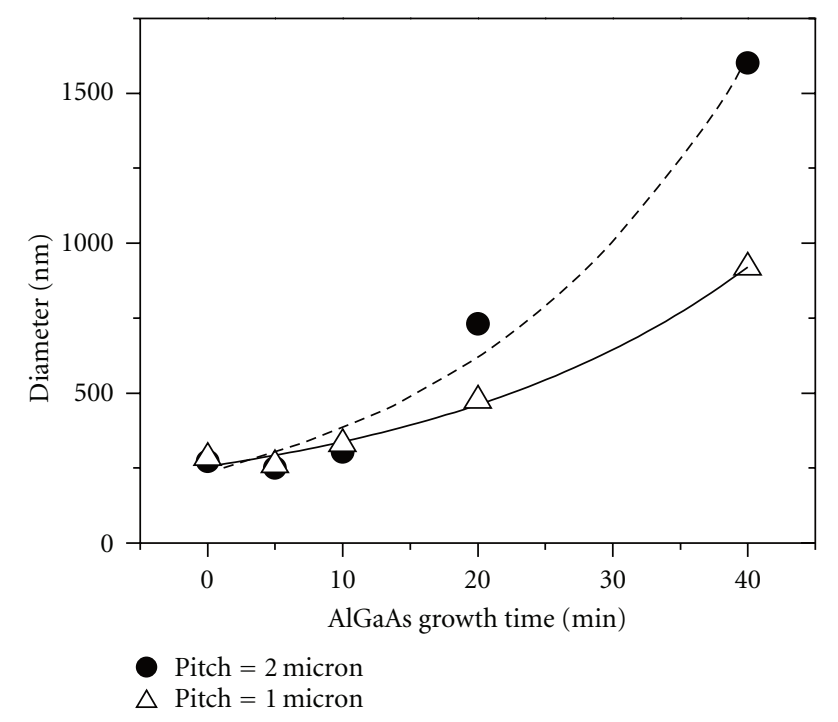

FIGURE 18: Dependence of nanowire thickness on AlGaAs growth time. Initial GaAs nanowire diameter was $270 \mathrm{~nm}$. Nanowire diameter increased with AlGaAs growth time [29].

To obtain the results given in Figure 18, we conducted an experiment on growing GaAs nanowires using larger mask openings and pitches to minimize the effect of irregular mask opening sizes than those used to grow the nanowires shown in Figures 17(a) and 17(b). The diameter of GaAs nanowires we obtained in this experiment was $270 \mathrm{~nm}$, and their typical size fluctuations were on the order of several percent [20]. We found that the amount of AlGaAs lateral growth was nonlinear and depended on pitch $a$ of the mask openings. Furthermore, as mentioned earlier, the amount of lateral growth for a 10 -min growth time was estimated to be 75-100 nm for the nanowires when $d=50$ $80 \mathrm{~nm}$, whereas it was negligible in the results presented in Figure 18. These findings mean that the lateral growth rate of AlGaAs is critically dependent on the size and arrangement of nanowires. Further studies are needed to clarify the details of the growth process.

Next, we conducted $\mu$-PL measurements of GaAs and GaAs/AlGaAs nanowire arrays at $290 \mathrm{~K}$. An He-Ne laser was used for excitation in this experiment, and the excitation intensity was $60 \mu \mathrm{W}$ (power density of about $1 \mathrm{~kW} / \mathrm{cm}^{2}$ ). Figure 19 shows the $\mu$-PL spectra of GaAs and core-shell nanowires. The average diameters of the nanowires are $80 \mathrm{~nm}$ for GaAs and $300 \mathrm{~nm}$ for the core-shell, and the pitch of the array is $0.4 \mu \mathrm{m}$. A PL spectrum of a semi-insulating (S.I.) GaAs substrate has also been plotted for reference. We can see some differences between the two types of nanowires and the reference. First, the PL intensity of the core-shell nanowires was much stronger than that of bare GaAs nanowires by a factor of about 20, and almost equal to that of the S.I. GaAs substrate. The weak emission from GaAs nanowires can be explained by the nonradiative recombination of photoexcited carriers at the air-exposed GaAs sidewall surface where there is a high density of surface states. The recovery of PL intensity in core-shell structures can also be explained by the

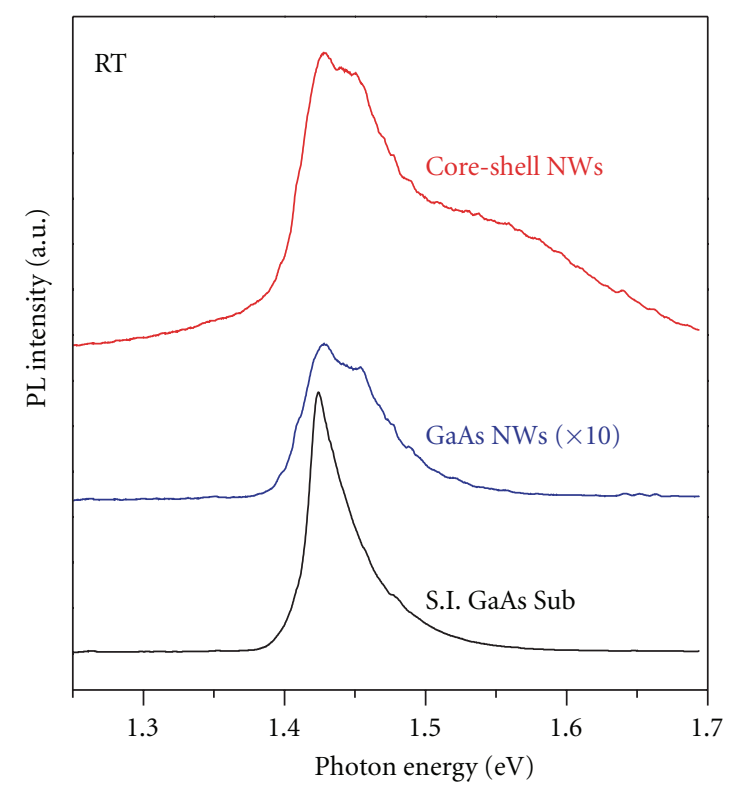

FIgURE 19: PL spectra of core-shell nanowires (NWs), bare GaAs nanowires, and reference semi-insulating GaAs substrate. Intensity of core-shell nanowires is about 20 times larger than that of bare GaAs nanowires [29].

surrounding $\mathrm{AlGaAs}$ barrier that prevents the coupling of photoexcited carriers in GaAs with the surface states [59, 60]. These results are consistent with our previous reports on GaAs/AlGaAs nanowires [20] and provide clues to obtaining high-quality and optically active nanowires. Second, when we compared the PL spectra of GaAs nanowires with that of GaAs/AlGaAs core-shell nanowires and with the S.I. GaAs substrate, we attributed the emissions at $1.45 \mathrm{eV}$ in both nanowires to the band-edge emission of GaAs and we found that the emissions indicated a blue shift of $30 \mathrm{meV}$ from the emission of the S.I. GaAs substrate. In circular nanowires surrounded with infinitely high potential barriers, the ground-state quantized energy associated with lateral confinement is given by $\left(2 \hbar^{2} \lambda_{01}^{2}\right) /\left(m d^{2}\right)$, where $m$ is the effective mass and $\lambda_{01}$ is the first zero of the zeroth-order Bessel function. For a GaAs core of $80 \mathrm{~nm}$, the expected blue shift is only $2.5 \mathrm{meV}$, which is much smaller than the observed $30 \mathrm{meV}$. The possible origin of the shift may be strain [61] or other mechanisms. A more careful study is required to clarify the origin of the blue shift of PL peaks in nanowires. Third, a broad feature on the high-energy side of GaAs was observed in core-shell nanowires when we compared the PL spectrum of the two types of nanowires. We think this is an emission from the AlGaAs shell and its spectral broadening indicates a broad distribution in Al composition. Estimating the aluminum content from the high-energy shoulder of the spectrum reveals that it is distributed approximately from $4 \%$ to $16 \%$, while the PL and $\mathrm{X}$-ray diffraction measurements of AlGaAs grown on planar (111)B indicate the $\mathrm{Al}$ content to be $12 \%$. The origin of the tendency for slightly less $\mathrm{Al}$ to be incorporated into nanowires is probably due to the difference in the migration 
length between Ga and Al during SA-MOVPE. Furthermore, as we previously reported [37], since the growth rate of GaAs depends on the nanowire diameter, there is a probability that the growth and $\mathrm{Al}$ incorporation rates in AlGaAs will change as the diameter increases due to lateral growth. This could give rise to nonuniform aluminum content in nanowires. In addition, the compositions of aluminum in AlGaAs between the sidewalls and that on top surfaces are possibly different.

\subsection{InP/InAs/InP Core-Multishell Structure and InAs Quan-} tum Tube [31]. We investigated the fabrication of an innovative core-multishell nanowire that was designed to embed a single strained radial quantum well (QW) in a higher band gap nanowire. The InP/InAs material system with a lattice mismatch of $3.2 \%$ was chosen for the present work. InP/InAs-strained QWs have tremendous potential for long wavelength optoelectronic applications because of their flexibility in enabling the electronic band structure to be tailored by using the combined effects of quantum confinement and strain. There is a schematic of the proposed core-multishell nanowire in Figure 20(a). The layer structure consists of an inner InP core and InAs and InP inner and outer shells, in which the InP core and the outer InP shell serve as the barrier layers while the InAs shell is the strained QW layer. Since the active layer, which is the InAs-strained QW, is in the form of a hollow cylindrical tube, this nanostructure can be deemed to be a quantum nanotube and is likely to exhibit exceptional properties as it is a two-dimensionally (2D) confined system with a three-dimensional (3D) cylindrical symmetry within a one-dimensional (1D) nanowire structure. It is extremely challenging to fabricate a lattice mismatched nanowire with a core-multishell heterostructure because of several complex requirements. Despite the lattice mismatch, InAs 2D layerby-layer growth should essentially occur on the InP nanowire core and also be preferentially along the lateral direction. Further, the fact that the inner core and the outermost shell are composed of the same material, InP, intrinsically necessitates InP to be grown axially for the inner core and laterally for the outer shell. The core-multishell nanowires reported earlier based on Si/Ge [62] and GaN/InGaN [63, 64] have been synthesized by a different approach, where the nanowire core was grown with the catalyst-assisted vaporliquid-solid (VLS) method and the shells were formed on the nanowire surface by vapor phase deposition. Unlike such a synthesis, our work involves growing both the core and the shells with a pure epitaxial method without the assistance of any catalysts. Critical factors are to accurately control the fabrication process, have precise knowledge about the growth mechanism, and control the direction of growth.

The source materials were TMI, TBP, and $5 \% \mathrm{AsH}_{3}$ in hydrogen. The core as well as the shells were grown sequentially as a single-continuous process. The growth conditions for an InP core and InAs shell have been described in detail elsewhere $[65,66]$. Subsequent to the growth of InAs, the conditions were changed yet again to grow the InP outer shell. While the growth of the inner InP core occurred along the top (111) direction, the outer InP layer should preferentially grow along the (110) direction. It is essential to control the direction of axial/radial growth of InP to achieve this complicated task. Phosphorus $(P)$ coverage governed by the growth temperature and TBP partial pressure was found to strongly influence the direction of InP growth [65]. While relatively lower $P$ coverage induced axial growth, higher $P$ coverage influenced lateral growth. Thus, as $P$ coverage was altered, competing growth of the top (111)A surface and the (110) sidewall plane occurred and it was possible to exactly define the direction of growth by precisely choosing the conditions. A growth temperature of $600^{\circ} \mathrm{C}$ and a high TBP partial pressure of $5.5 \times 10^{-3}$ atm were the optimum conditions that favored the lateral growth of InP over InAs sidewall facets. The single-step growth process under these conditions ultimately resulted in the successful fabrication of nanowire arrays with an InP/InAs/InP core-multishell heterostructure.

Our SEM studies clearly indicated that the grown structures were well defined (Figure 20(b)). The length of a typical core-multishell nanowire was about $2.5 \mu \mathrm{m}$, and its diameter was about $140 \mathrm{~nm}$. The grown nanowires were subjected to anisotropic dry etching followed by stain etching to analyze their cross-sectional features. The diameter of the inner InP core and the thickness of the outer InP shell covering it were 70 and $30 \mathrm{~nm}$, respectively. The InAs QW layer exhibited a well-defined hexagonal cross section, and the well width was found to be $5 \mathrm{~nm}$ (inset of Figure 20(b)). The most remarkable feature of this nanostructure is that the dimensions as well as the layer structure thickness could be accurately defined by growth conditions. Thus, precise control over the fabrication process gave us the freedom to engineer the physical properties determined by the shape, size, and QW width of the nanostructure. Further, selectivearea growth enabled the fabrication of these nanowires to be guided in a highly periodic manner. The successful fabrication of this core-multishell nanowire is extremely significant as all three layers are single crystalline and these were epitaxially grown without the presence of any catalysts. Coremultishell nanowires with different InAs QW widths were grown by varying the InAs growth time to enable further characterization.

Four K $\mu$-PL measurements were carried out on the coremultishell nanowires with an InAs well width of $1.5 \mathrm{~nm}$ using the 532-nm continuous-wave output of a diode pumped $\mathrm{Nd}: \mathrm{YVO}_{4}$ laser for excitation. The excitation beam was focused onto a $2 \mu \mathrm{m}$-diameter spot with a $\times 20$ microscope objective on the sample, which had been placed in a cold finger cryostat. The PL collected through the same microscope objective was dispersed into a spectrometer with a liquid-nitrogen-cooled InGaAs photomultiplier tube. The PL spectra of core-multishell nanowires with different pattern periods are shown in Figures 21(a) and 21(b). Spectrum (a) shows PL from a nanowire array with a $3-\mu \mathrm{m}$ period, whereas (b) is from an array with a $0.4-\mu \mathrm{m}$ period. Since the $3-\mu \mathrm{m}$ period is greater than the spot size, spectrum (a) can be concluded to be from a single core-multishell nanowire [67]. Spectrum (a) consisted of two distinct emission peaks. The PL peak observed at an energy of $0.861 \mathrm{eV}$ is due to an InAs QW formed on the (110) sidewalls of InP nanowires, while the peak at $1.401 \mathrm{eV}$ corresponds to the InP barrier, as will be 


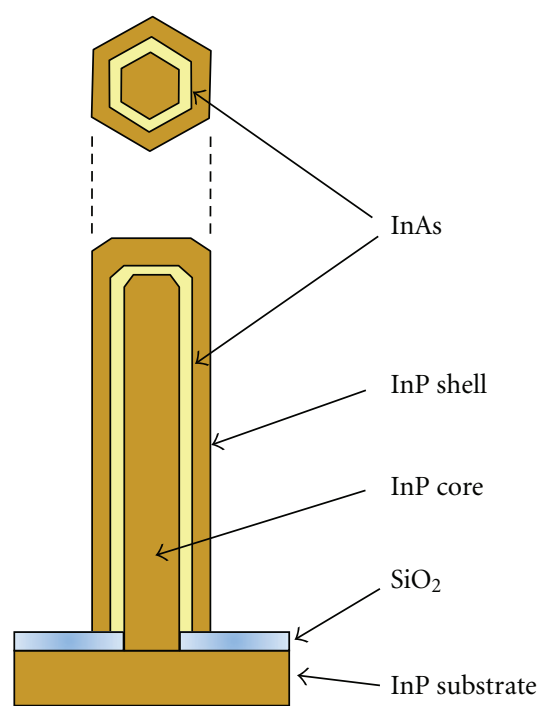

(a)

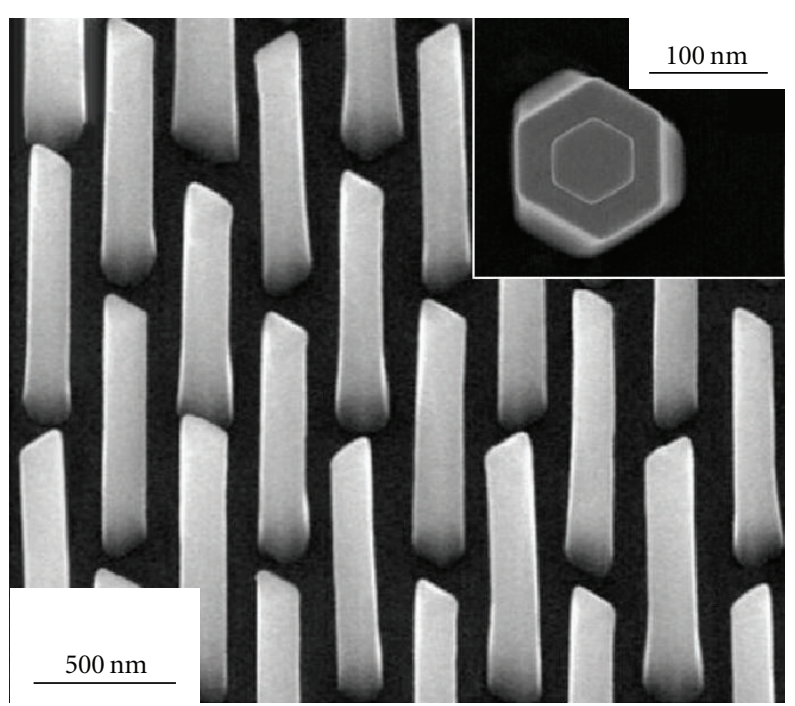

(b)

FIGURE 20: Cross-sectional structures and SEM images of nanowires. (a) Schematic of cross-sections of InP/InAs/InP core-multishell nanowire. (b) SEM image of periodically aligned InP/InAs/InP core-multishell nanowire array. Inset is top view of high-resolution SEM image of core-multishell nanowire observed after anisotropic dry etching and stain etching [31].

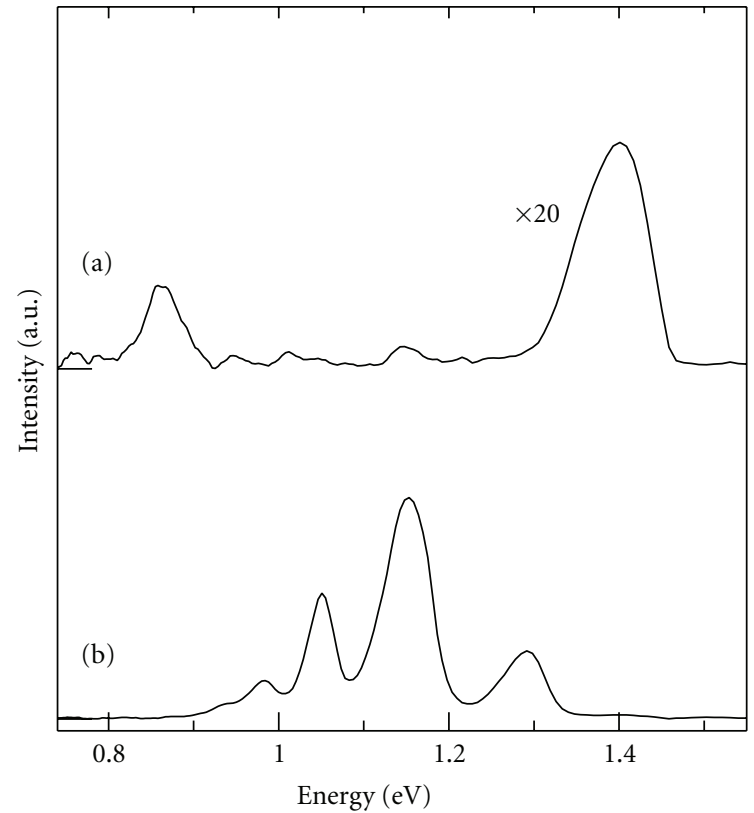

FIGURE 21: 4-K PL spectra of nanowire arrays with (a) 3- and (b) $0.4-\mu \mathrm{m}$ periods $[31]$.

discussed later in detail. Further, the InAs layer on top of the InP core is rather thick and does not have any influence on the observed PL spectra. Spectrum (b), taken from multiple core-multishell nanowires (30 nanowires, considering the size of the spot), on the other hand, exhibits multiple peaks at around $0.86-1.45 \mathrm{eV}$, which are also due to the emissions from InAs radial QWs.
To confirm these peak assignments, the ground-state transition energy in strained InAs QWs on InP (110) was calculated using a simple square well potential. The parameters for calculation were determined as follows. Initially, offsets for conduction and heavy hole bands were calculated based on model solid theory [68] taking into account strain in InAs on InP (110). While the unstrained isotropic effective mass was used for electrons, the heavy hole effective mass $\left(m_{\mathrm{hh}}\right)$ was determined based on

$$
m_{\mathrm{hh}}=\frac{m_{0}}{\left(\gamma_{1}-\left(\gamma_{2}^{2}+3 \gamma_{3}^{2}\right)^{1 / 2}\right)},
$$

where $m_{0}$ is the free electron mass and $\gamma_{1}, \gamma_{2}$, and $\gamma_{3}$ are the Luttinger parameters. The heavy hole effective mass was obtained using a $4 \times 4$ Luttinger-Kohn Hamiltonian with the quantization axis along the (110) direction [69]. The values of the deformation potential and Luttinger parameters were taken from Chuang [70] and Sugawara et al. [71]. The present calculation considerably simplifies the treatment of nonparabolicity in the conduction band and complex valence band structure of strained (110)-oriented QWs. A more accurate account on the electronic structure in a strained core-multishell nanowire will be reported elsewhere, but the accuracy of the present theoretical treatment is expected to be within the ambiguity of the parameters, the difference in the crystallographic structure [66] of InP and InAs, and effective mass approximation. The results of the calculation have been plotted as a function of the InAs well width (Figure 22). According to these results, the PL peak at $0.861 \mathrm{eV}$ observed for a single core-multishell nanowire corresponds to a well width of $2.08 \mathrm{~nm}$, which reasonably agrees with the measured thickness of the InAs layer. Further, this peak is relatively broader with a full width at half maximum (FWHM) of $48 \mathrm{meV}$, which can be mainly 


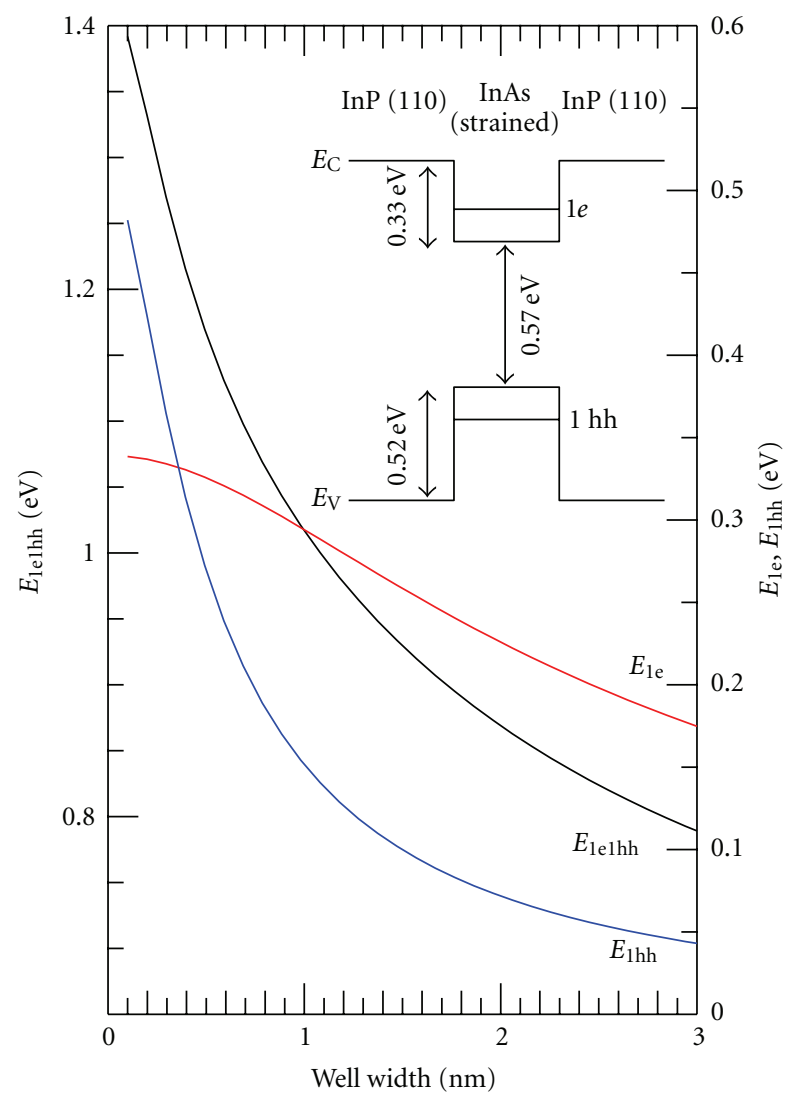

FIGURE 22: Plot of ground-state transition energy as function of well width for InP/InAs/InP strained QW on InP (110) [31].

attributed to the fluctuations in well width, although it may be in part due to inhomogeneous strain in the InAs layer. Also note that the PL peak of InP has slightly red shifted compared to the bare InP nanowires [65]. Our preliminary calculations suggest that this shift is not due to the tensile strain in InP nanowires but it bears further investigation. In spectrum (b) the peaks at $0.984,1.05,1.154$, and $1.292 \mathrm{eV}$ correspond to InAs QW widths of 1.16, 0.856, 0.532, and $0.262 \mathrm{~nm}$. A minimum width for InAs in InP is accomplished by substituting one layer of $P$ with As, and this corresponds to a half monolayer thickness for (110). Thus, the thinnest InAs QW in the elastic continuum model is expected to be $0.219 \mathrm{~nm}$, and the well width should be its integer multiples. Considering the accuracy of the present model, these results reiterate the formation of InAs QWs on the side walls of InP nanowires and the multiple peaks indicate that there might be monolayer QW thickness variations in individual nanowires in an array. The peak broadening, in this case also, can be partly attributed to inhomogeneous strain in the InAs layer in the nanowire array. Further, the InAs layer thickness is less in nanowires with a $0.4-\mu \mathrm{m}$ period than that in nanowires with a $3-\mu \mathrm{m}$ period. This difference in the InAs layer thickness may be attributed to the gas-phase diffusion of In, where the lateral growth rate of InAs on the sidewalls is much higher for smaller densities of nanowires, that is for nanowire arrays with larger periods. However, as this kind of diffusion model cannot fully account for the vertical growth rate of nanowires, it thus requires more detailed investigations.

The band offset of the lowest energy levels for the conduction band and the valence band of the InP/InAs/InP core-multishell nanowire depicted in the inset of Figure 22 indicates a heterostructure with Type-I alignment, that is, the bottom (top) of the InAs conduction (valence) band is at a lower (higher) energy level of the conduction (valence) band bottom (top) of InP. Recent investigations, using timeresolved and spectrally resolved PL and PL excitation measurements, of the InP/InAs/InP core-multishell nanowires revealed the possibility of a Type-II heterostructure, that is, band alignment with the conduction (valence) band of InAs lying $0.16(0.32) \mathrm{eV}$ above the conduction (valence) band of $\operatorname{InP}[72]$.

4.3. GaAs/GaAsP Core-Shell Structure and Laser Emission [32]. Semiconductor subwavelength nanowires have been demonstrated in recent years to have laser emissions [7379]. Representative semiconductor materials for fabricating nanowire lasers involve $\mathrm{ZnO}, \mathrm{GaN}$, and $\mathrm{CdS}$. Such nanowire lasers are currently among the smallest known lasing devices, with lengths between 1 and several tens of micrometers and diameters that can be significantly smaller than the emission wavelength in a vacuum. Because of the large dielectric contrast between the nanowire and the ambient in this size range, strong lateral optical confinement is created. The end facets of a single-crystalline nanowire form a natural mirror surface that creates an axial resonator. That is, onedimensional semiconductor nanowires act not only as a gain medium but also as a waveguide and a Fabry-Perot resonator, which provide coherent feedback. The light-emitting capability of the nanowires, combined with their other unique features that arise due to their one dimensionality, makes them particularly interesting to consider as candidates for components in future nanoscale photonic systems. However, most advances in nanowire lasers have successfully been achieved through wide-bandgap semiconductor materials, which provide ultraviolet-to-blue laser emissions. To date, limited investigations into nanowire lasers in the nearinfrared (NIR) spectral range have been reported [80], while there are vast areas of applications in NIR lasers particularly for optical fiber communications. For instance, lasers operating at $850 \mathrm{~nm}$ can be used in in-house and in-enterprise local area networks with extremely high transmission bit rates. In this case, GaAs-based materials are primarily important while the main difficulty in attaining nanowire lasers in these material systems is that the high density of surface states will lead to strong decay of emission intensity in GaAs nanowires.

We here describe GaAs/GaAsP coaxial core-shell nanowires fabricated by SA-MOVPE and report our observations that a single wire has optically pumped NIR lasing emissions. GaAs and GaAsP were grown in succession for core-shell heterostructures. The partial pressures for GaAs were $1.0 \times$ $10^{-6}$ atm for TMG and $2.5 \times 10^{-4}$ atm for $\mathrm{AsH}_{3}$. The growth temperature and growth time were set to $750^{\circ} \mathrm{C}$ and $60 \mathrm{~min}$. The partial pressures for growing the GaAsP shell 
corresponded to $1.0 \times 10^{-6}, 2 \times 10^{-4}$, and $2.5 \times 10^{-4}$ atm for TMG, TBP, and $\mathrm{AsH}_{3}$. The growth temperature and growth time were $650^{\circ} \mathrm{C}$ and $5 \mathrm{~min}$. Perfect selective epitaxy of both GaAs and GaAsP was achieved under these conditions.

As-grown nanowires were mechanically cut down and dispersed onto $2-\mu \mathrm{m}$ thick $\mathrm{SiO}_{2}$-covered $\mathrm{Si}$ substrates to optically characterize single nanowires. PL measurements were carried out using a regeneratively amplified Ti:sapphire pulsed laser (140-fs pulse duration and $78-\mathrm{MHz}$ repetition rate) with a wavelength of $753 \mathrm{~nm}$. The excitation beam was focused with a $\times 50$ microscope objective $(\mathrm{NA}=0.42)$ onto a spot of about $2 \mu \mathrm{m}$ in diameter on a sample in a cryostat. The emissions through the same microscope objective were collected into a liquid-nitrogen-cooled CCD for spectral analysis or a CCD camera for imaging.

First, the overall morphology of as-grown nanowires was examined by SEM. A schematic cross-section of a GaAs/GaAsP nanowire and typical SEM images of GaAs nanowires and $\mathrm{GaAs} / \mathrm{GaAsP}$ core-shell nanowires are shown in Figures 23(a) to 23(c). A uniform array of vertical standing nanowires was prepared with a smooth top surface and sidewall. Close-up SEM images of nanowires indicate they have a clear hexagonal shape. As the wires grew perpendicularly on GaAs (111)B substrates, the growth direction thus coincided with the (111)B direction. Since this SA-MOVPE method fully utilized the nature of epitaxial growth and used no gold as a seed for the nanowires, they were synthesized with superior crystalline quality at atomic precision. The nanowire diameters, $d$, in this study were controlled, so they ranged between 200 and $500 \mathrm{~nm}$ and their lengths ranged between 2 and $6 \mu \mathrm{m}$. The nanowire sizes depended on the prepared mask openings on the substrate and growth conditions $[37,81]$. The relatively larger nanowire diameters in this experiment were necessary to enhance reflectivity at both ends of the nanowire surface and bring sufficient optical confinement for lasing, especially at NIR wavelengths. SEM images (b) and (c) in Figure 23 show that the length of the core-shell wire is almost equal to that of GaAs, but the diameter of the core-shell wire is approximately $100 \mathrm{~nm}$ greater than that of the GaAs wire, suggesting a 50-nm thick GaAsP shell around the GaAs core wire.

TEM and EDX spectroscopy (EDX) were then applied to examine the structure of nanowires and their atomic composition. A bright-field TEM image and EDX line scans obtained from the axial plane of a single nanowire exposed by focused ion beam (FIB) etching are shown in Figures 24(a) to 24(c). Even though the heterointerface of GaAs and GaAsP cannot clearly be distinguished in the bright-field and high-resolution TEM images, the EDX line scan clearly shows the formation of core-shell structures. The green, blue, and red lines in Figures 24(b) and 24(c) correspond to the EDX counts of elemental Ga, As, and $P$. The length of this nanowire was $2.8 \mu \mathrm{m}$, and its diameter was $390 \mathrm{~nm}$. In Figure 24(b), the results are presented for an EDX line scan along the axis of the nanowire (along the solid white arrow in Figure 24(a)). Here the right end of the nanowire corresponds to its top end during growth. Another line scan performed across the diameter of the nanowire is shown in Figure 24(c). The scan position is labeled with a blue solid arrow in Figure 24(a). The axial EDX scan indicates that the nanowire core is entirely composed of GaAs along the center axis, and the $P$ content on its top surface is negligible. However, the radial line scan clearly designates the presence of a $P$ element around the GaAs core. These results prove that the nanowire structure should be a GaAs core surrounded by a GaAsP shell. The $P$ concentration in the GaAsP shell is around $10 \%$ in atomic content, that is $\mathrm{GaAs}_{0.8} \mathrm{P}_{0.2}$, and the thickness of the outer GaAsP shell is estimated to be $50 \mathrm{~nm}$, which is consistent with that SEM observations (Figures 23(b) and 23(c)) before and after the growth of GaAsP.

Fabry-Perot microcavity modes were observed in single GaAs nanowires [82] in our previous study. The PL spectra results suggested that a Fabry-Perot microcavity was formed along the length of the nanowire, and the (111) facets of both ends acted as reflecting mirrors. However, due to the nonradiative recombination of photoexcited carriers on the air-exposed GaAs sidewall surface where there is high density of surface states, the emission intensity of bare GaAs wires is too weak to obtain lasing. Core-shell nanowires are expected to be used instead of bare GaAs wires to further optimize this cavity quality. The function of the shell layer is to passivate the surface states of the GaAs core nanowires, resulting in high-quality and optically active nanowires. The $\mathrm{PL}$ spectra of the GaAs/GaAsP core-shell nanowires in this study displayed apparently strong emission intensity, which was stronger than that of bare GaAs nanowires by a factor of over two orders of magnitude. A shell, $50 \mathrm{~nm}$ in thickness, should have been sufficient in this study to passivate surface states since it has been reported that a shell of around $10 \mathrm{~nm}$ in thickness suppressed the reduction of PL efficiency $[59,60]$.

An isolated single GaAs/GaAsP core-shell nanowire was then excited with a pulsed laser, and PL emissions were subsequently collected at $4.2 \mathrm{~K}$, as can be seen in Figures 25(a), 25(b), and 25(c). This wire is $330 \mathrm{~nm}$ in diameter and $5.5 \mu \mathrm{m}$ in length. The PL spectra at low excitation power densities display a broad emission band at about $820 \mathrm{~nm}$. However, above a certain threshold $\left(8.4 \mathrm{~kW} / \mathrm{cm}^{2}\right.$ in this nanowire), a sharp and narrow peak centered at about $816 \mathrm{~nm}$ appears and the peak intensity increases rapidly with excitation energy. This emission peak is exactly consistent with the GaAs band gap energy $(1.52 \mathrm{eV}$ at $4.2 \mathrm{~K})$, indicating the onset of stimulated emissions from the GaAs core of the nanowire. From Figure 26(a), stimulated emission or lasing can be evidenced by the appearance of simultaneous line narrowing and a superlinear increase in intensity at pump densities above the threshold. However, as the pump power density is further increased above $15.0 \mathrm{~kW} / \mathrm{cm}^{2}$, the emission intensity saturates, indicating that gain is pinned. We speculated on what contributed to the quick saturation of laser emissions with the present experimental configuration. That is, we used a tight focus for the incident laser on the nanowire, where the diameter of the laser beam was around $2 \mu \mathrm{m}$, which is much less than the wire's length. These led to a smaller pumping area in the nanowire; thus, gain was only obtained in the vicinity of the excitation region. As a result, gain quickly saturated. Excitation-induced local heating may also be a reason for the quick saturation in gain. Moreover, the line 


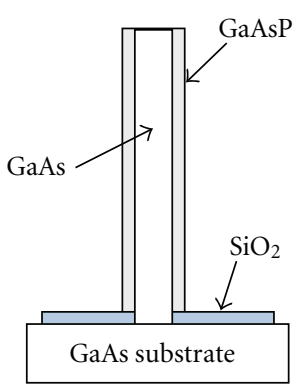

(a)

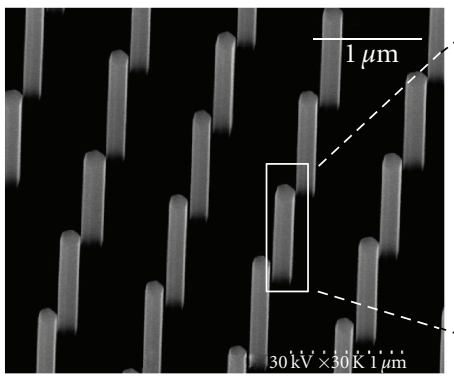

(b)

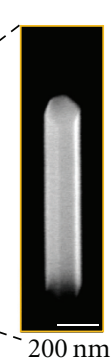

$200 \mathrm{~nm}$

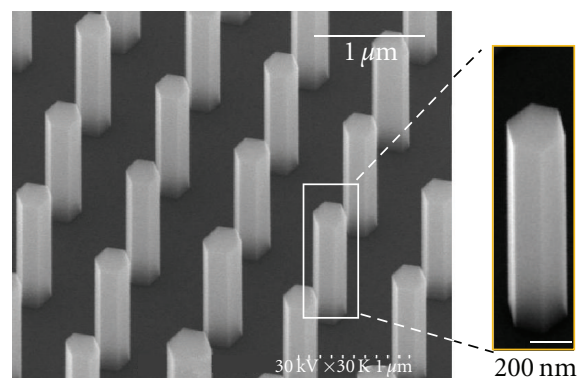

(c)

FIGURE 23: Schematic of structure and SEM images of nanowires. (a) Schematic of cross-sectional structure of core-shell nanowire. (b) and (c) SEM images of as grown GaAs and GaAs/GaAsP nanowires for comparison [32].

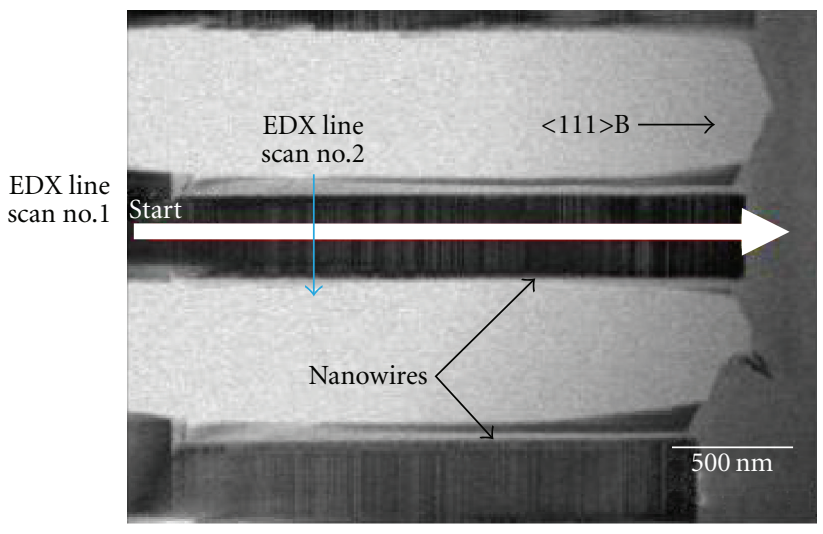

(a)

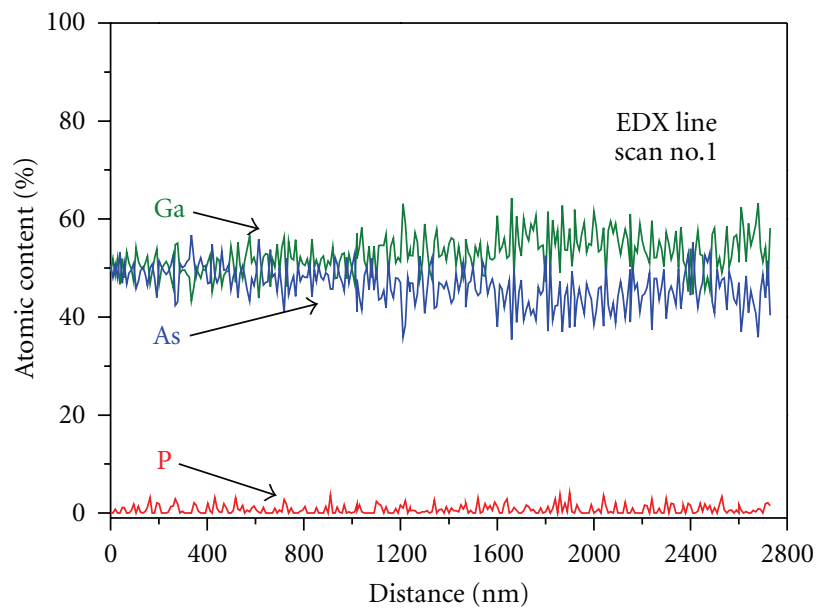

(b)

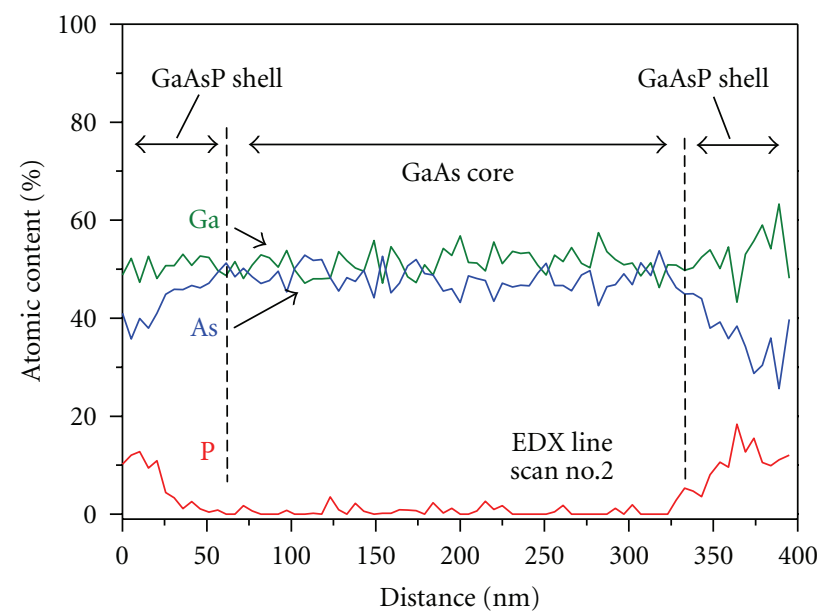

(c)

FIgURE 24: TEM/EDX analysis of GaAs/GaAsP nanowire. (a) Bright-field TEM image and axial/radial EDX line scans on nanowire. Axial plane of wire was exposed with FIB etching. (b) Variations in Ga (green), As (blue), and $P$ (red) content for axial line scan no. 1 in (a). (c) Variations in Ga, As, and P content for radial scan no. 2 in (a). Radial EDX scan indicates that GaAs core is surrounded by approximately 50-nm thick GaAsP shell [32]. 


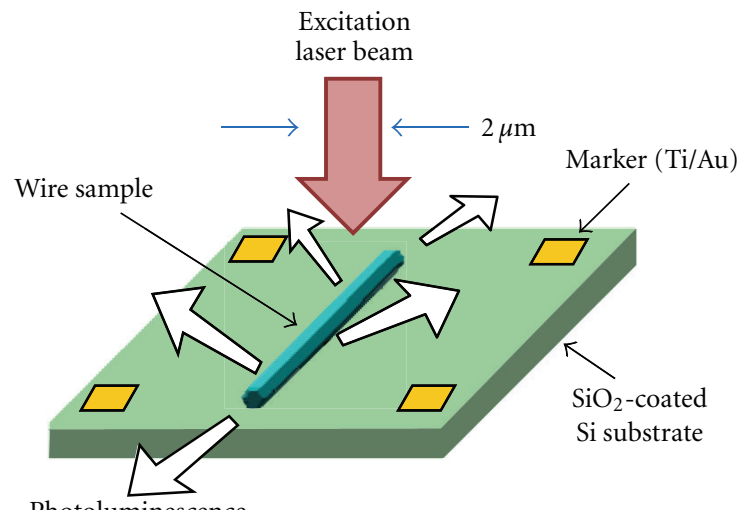

(a)

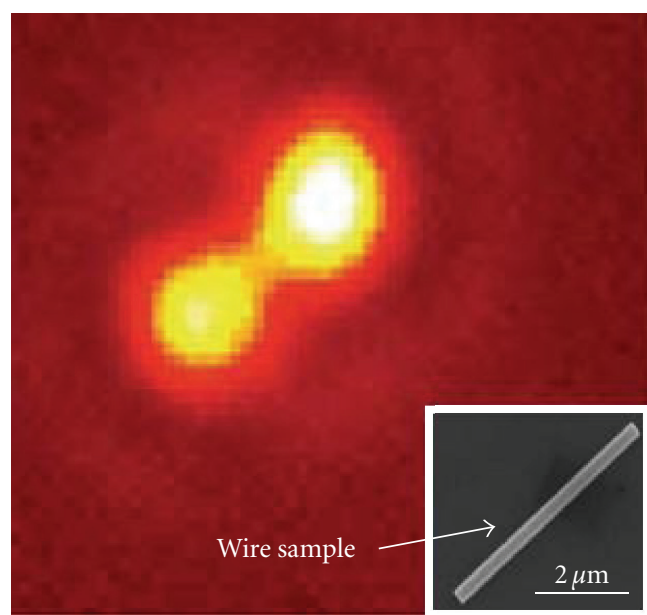

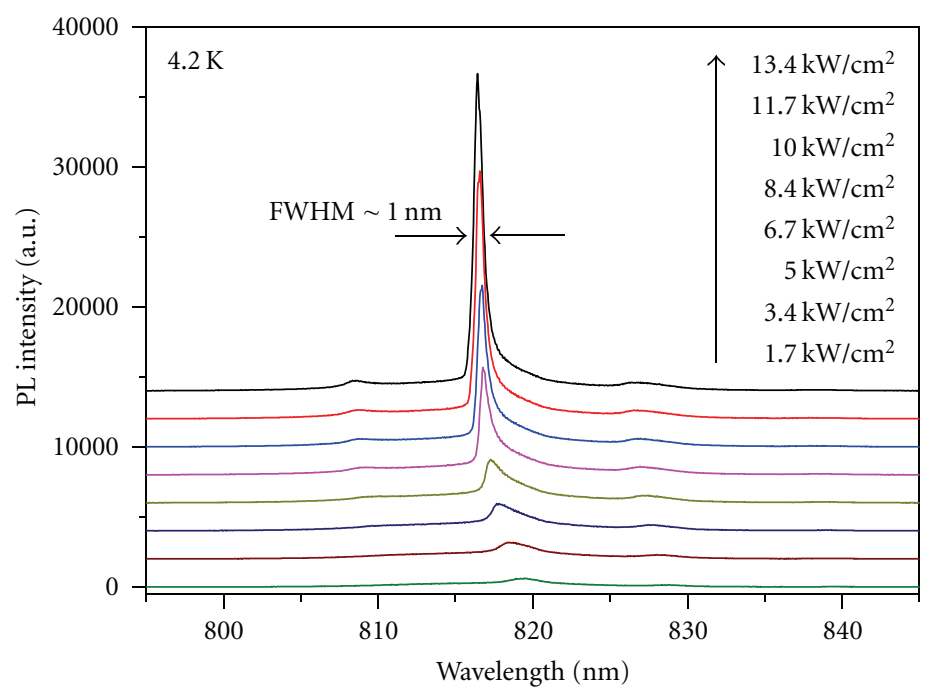

(c)

(b)

FIGURE 25: Images of optically pumped single GaAs/GaAsP core-shell nanowire lasers and PL spectra. (a) Schematic of nanowire sample excited by laser beam. (b) CCD camera image of luminescence from nanowire. Inset at bottom right is SEM image of 5.5- $\mu \mathrm{m}-\mathrm{long}$ nanowire. (c) PL spectra from single GaAs/GaAsP core-shell nanowire with length of $5.5 \mu \mathrm{m}$, as function of increasing excitation power density at $4.2 \mathrm{~K}$ [32].

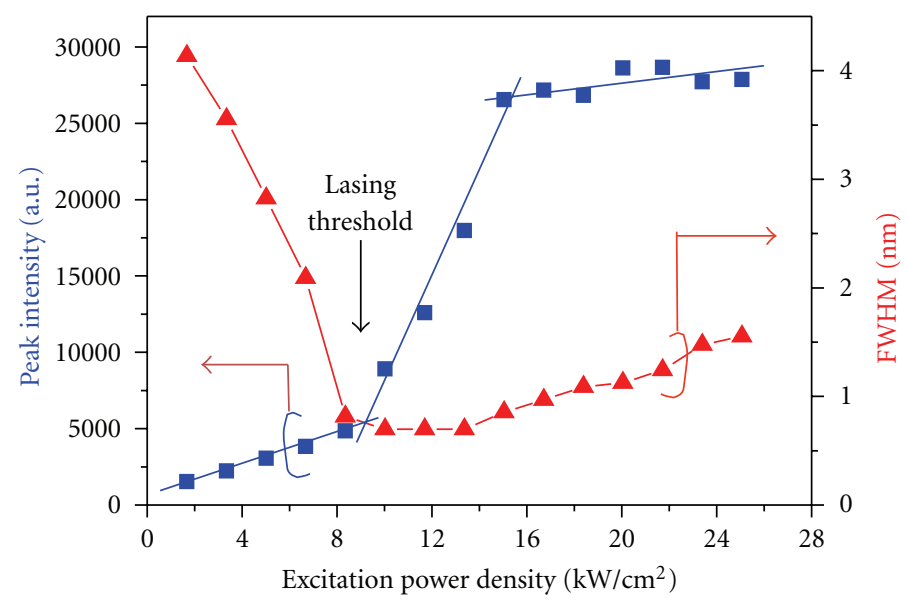

(a)

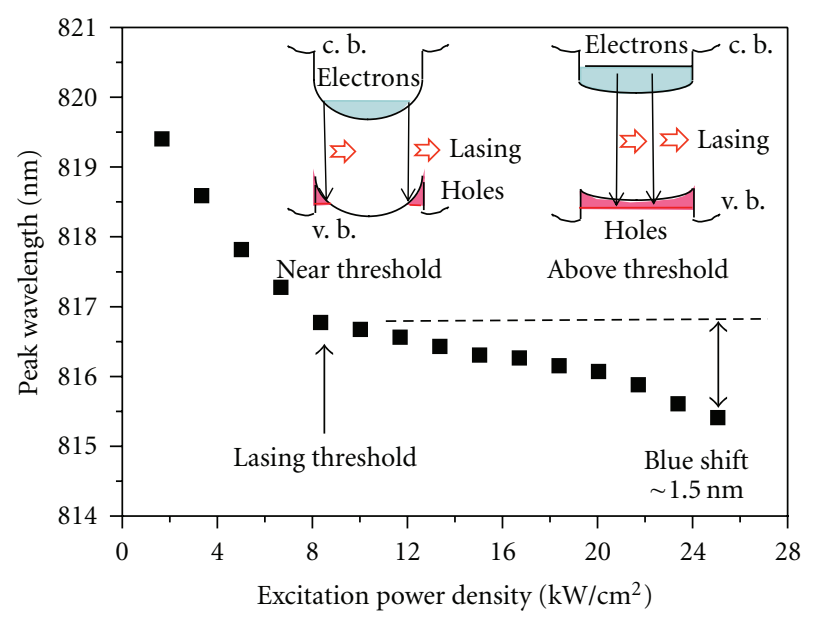

(b)

FIGURE 26: PL peak intensity/wavelength as function of excitation power density. (a) Plots of emission peak intensity (marks with filled squares) at center wavelength of $816 \mathrm{~nm}$ and full width at half maximum (FWHM) (marks with filled triangles) of PL spectra. (b) Plots of lasing peak wavelength versus excitation power density. Inset indicates how band bending of conduction band (c. b.) bottom and valence band (v. b.) top change as densities of accumulated photoexcited electrons and holes increase [32]. 


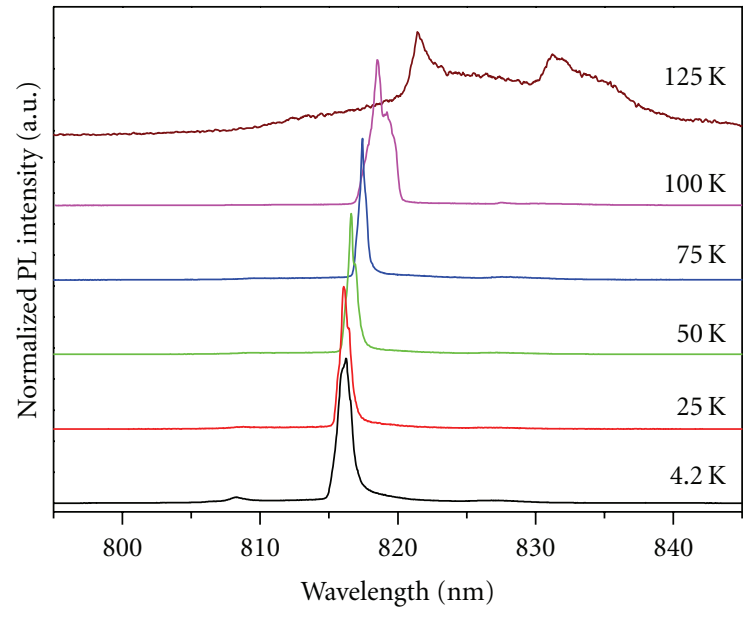

(a)

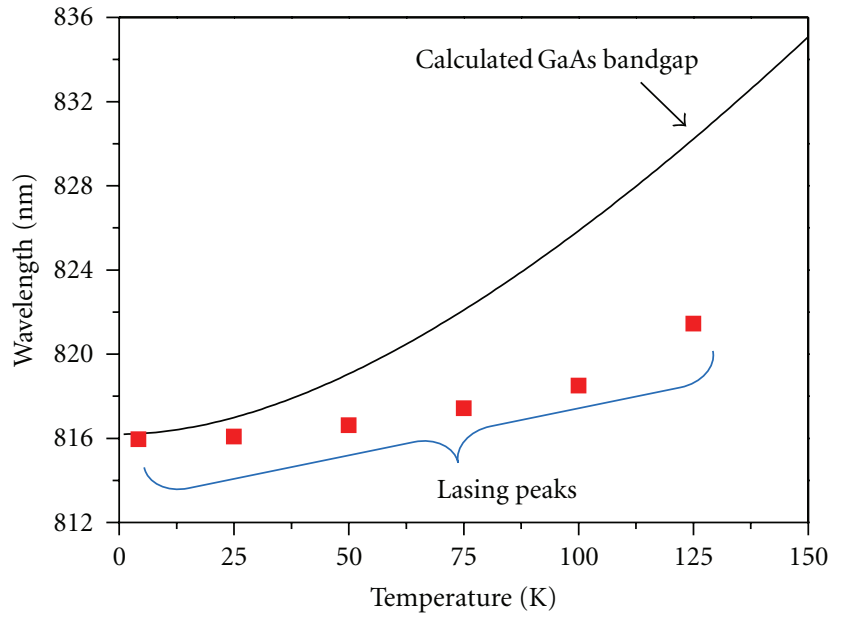

(b)

Figure 27: Normalized temperature-dependent spectral features recorded from single GaAs/GaAsP nanowire for lasing. (a) PL spectra for lasing recorded at 4.2, 25, 50, 75, 100, and $125 \mathrm{~K}$. (b) Plots of temperature-dependent lasing wavelengths (marks with filled squares). Dependence of temperature on GaAs bandgap is also given in curve for comparison [32].

width for conventional semiconductor lasers varies inversely with pump power, due to an increasing degree of population inversion. However, microlasers pumped with short intense pulses can exhibit additional nonequilibrium effects that act to broaden the lasing peak [83]. Such an effect can also be seen in Figure 26(a). The laser peak is broadened from 0.7 to $1.5 \mathrm{~nm}$ over the power range of 13.4 to $25.1 \mathrm{~kW} / \mathrm{cm}^{2}$. It is likely that there is increased spontaneous emission noise at higher pump intensities due to amplified spontaneous emissions that cause phase fluctuations that couple to the real and imaginary parts of the material susceptibility. These optical density fluctuations would couple to the cavity modes and act to broaden the lines, especially in regions with the highest dispersion and absorption.

As usual, nanowire lasing red shifts with increasing excitation power, due to the heating effect or the bandgap renormalization induced by the electron-hole plasma state [84]. In contrast, blue shift in the lasing peak of this wire can be observed due to an increase in the pump power as shown in Figure 26(b). The reason for this blue shift is unclear, but one possibility may be due to band bending $[85,86]$. According to the model, photoexcited charge carriers are generated and reach a dynamic equilibrium, where electrons get confined near the nanowire surface but holes accumulate near the nanowire center. Thus, an electron near the surface recombines with a hole near the center, leading to low transition energy, while at the high excitation intensity, the band bending effect weakens due to the formation of more charge carriers, bringing about high emission energy and transition probability. The nanowires used in the model are nondoped GaAs [85] and p-type InP [86]. However, the GaAs nanowires in our experiment were assumed to be $n$-type (carrier concentration of around $4 \times 10^{17} \mathrm{~cm}^{-3}$, obtained by electron Hall measurements of planar GaAs epitaxial layers grown on S.I. GaAs (001) substrates at the same temperature as the nanowires). Thus, the band bending and the accumulation of photoexcited charge carriers for the present $\mathrm{GaAs} / \mathrm{GaAsP}$ nanowires could be reversed compared to van Weert et al.'s model [86], as depicted in the inset of Figure 26(b), and the PL blue shift can be explained in the same fashion.

Furthermore, the normalized temperature-dependent PL spectra of the same nanowire were recorded from 4.2 to $150 \mathrm{~K}$, as shown in Figure 27(a). The excitation power density was set to $21.0 \mathrm{~kW} / \mathrm{cm}^{2}$ for all the spectra. The spectral features associated with lasing were expected to exhibit pronounced red shift with temperature. However, from Figure 27(b), compared to the temperature-dependent red shift in the GaAs bandgap energy, the red shift in the lasing wavelength is weak ( $\Delta \lambda$ is $2.5 \mathrm{~nm}$ from 4.2 to $100 \mathrm{~K})$. These results agree with the previous observation of the temperature-dependent cavity mode in single GaAs nanowires $(\Delta \lambda$ was $2-2.6 \mathrm{~nm}$ from 4.2 to $100 \mathrm{~K}$ ) [82]. Considering the expression of $m=2 n L / \lambda$, where $m$ is the longitudinal mode order, the peak position is strictly determined by refractive index $n$, for a certain nanowire with a length of $L$. With temperatures ranging from 4.2 to $100 \mathrm{~K}$, the temperature-dependent variations in the refractive index are weaker, leading to less change in the lasing peak with temperature.

The lasing characteristics of the GaAs core indicate that these heterostructure nanowires are very efficient gain media, which can be attributed to their ideal photon-confinement structures. Since the regular dimensions of the wires make them excellent waveguides, emission light can be accumulated and amplified through them being reflected from both ends of the wires to finally produce stimulated emissions or lasing under sufficiently high excitation. As a comparison, it must be emphasized that no lasing has been observed thus far for pure GaAs nanowires. The lasing in the present 
TABLE 1: Far-field optical images of lasing emissions from single GaAs/GaAsP core-shell nanowires. (a)-(c) Observed lasing emissions from different nanowires with lengths of 3.4, 4.5, and $5.5 \mu \mathrm{m}$. Incident laser spot is focused perpendicularly at center of nanowires. Scale bars: $5 \mu \mathrm{m}$. (d)-(f) Simulated interference patterns for corresponding nanowires in panels (a)-(c), assuming spherical nondirectional emissions from wire end facets [32].

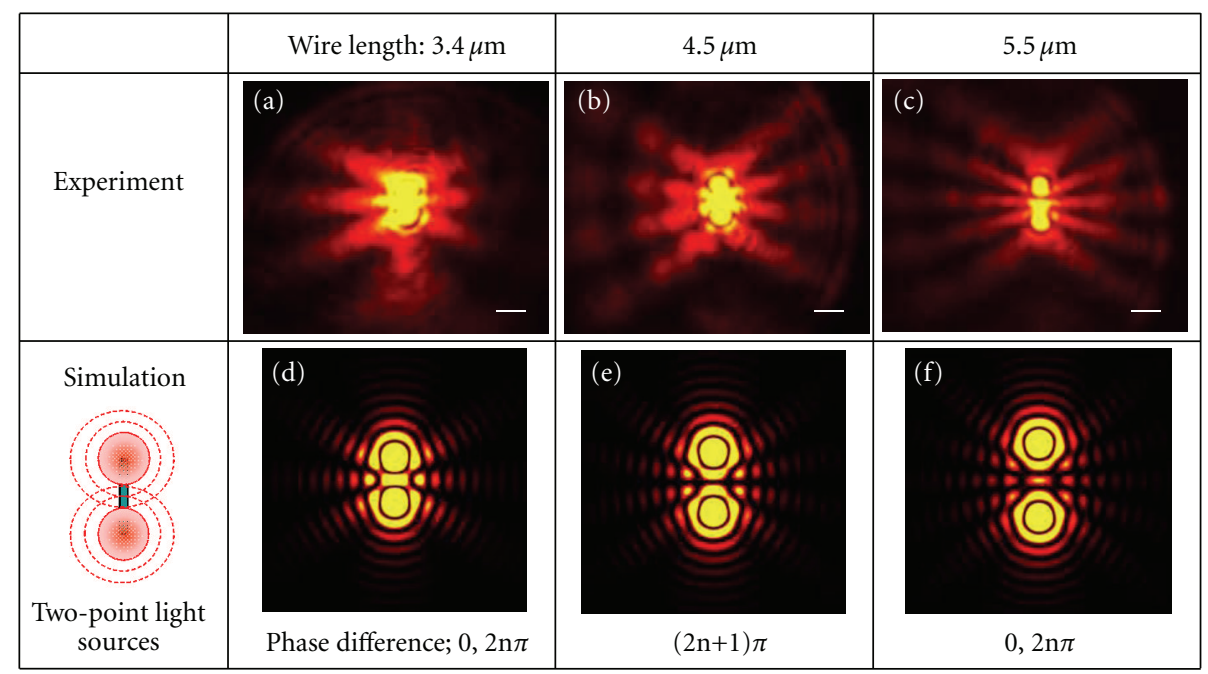

core-shell structures is mainly attributed to the reduction in nonradiative surface recombination as previously discussed. The influence of photon confinement due to index contrast between the core and shell is not important in the present structure because of the small refractive-index contrast (the refractive index at a $\lambda$ of $816 \mathrm{~nm}$ is 3.66 and 3.56 for GaAs and GaAsP, resp.) and thin shell thickness (about $50 \mathrm{~nm}$ ).

The top half of Table 1 shows lasing emission images from several core-shell nanowires of different lengths. The images were recorded with a laser filter when the excitation laser was focused at the center of the nanowires. All wires exhibit very bright luminescence spots that can be observed at both ends. This is a typical feature of an optical waveguide, and it suggests that the wires are able to absorb excitation light and propagate PL emissions toward the ends. Almost all the core-shell nanowires exhibited this kind of waveguide behavior. Since wave-guided light is generated from PL within the nanowires, these wires can be classified as active waveguides instead of passive ones where light must be coupled-in from external sources. Interestingly, intensity modulations around the wires can be observed, suggesting that diffraction and interference occur, which originate from spherical emissions from both wire end facets with a difference in fixed phase. These interference patterns are expected to depend on the wire length. The longer nanowires obviously exhibit more complex interference patterns, compared to the shorter ones. We simulated diffraction and interference patterns to enable more quantitative understanding following van Vugt et al.'s approach [87] (assuming spherical nondirectional emissions in nanowire end facets), which are presented in the bottom half of Table 1. Here, the NA of the microscope objective and the difference in the phase at two edges have been taken into account. We can see that the interference patterns around the nanowires are nicely reproduced for all three nanowires.
The next issue with GaAs/GaAsP core-shell nanowires is to develop an electrode fabrication process to enable current injection to the nanowires, where light from them should be extracted without being blocked or absorbed by electrode metal. Heat dissipation from nanowires during current injection is another issue that needs to be solved to enable stable operation of devices at room temperature (RT). The active layer in commercially available semiconductor lasers is buried in a semiconductor material with a greater energy bandgap than the active layer. Therefore, buried heterostructures should be considered to dissipate heat from nanowire LEDs to allow their practical use.

\section{Outlook for the Future}

The mitigation of mechanical strain and curbs to the development of crystal dislocations at the junction interface of lattice mismatching systems are advantages of using nanowires. Reducing the number of defects should effectively enable semiconductor nanowires to be fabricated on a $\mathrm{Si}$ platform, which is promising for integrating them with ICs or using them in large-scale production to achieve low cost. Important factors to consider in practical use in lighting and display systems for optical information processing where LEDs are used as optical sources are control over their emission wavelength and optical power and the reliability of devices. Lattice-mismatched heterostructure epitaxial layers, for example GaInAs/GaAs, GaInP/GaAs, and GaInN/GaN grown on a III-V semiconductor substrate or on a sapphire substrate, have been used to control the emission wavelength of LEDs. Material costs for the substrates would be reduced if $\mathrm{Si}$ was employed instead of expensive substrates [8892]. However, as previously mentioned, the heterostructures grown on Si are subject to mechanical strain and crystal 
dislocations at the substrate interface due to lattice mismatching. The dislocations developed in the active region of devices are detrimental to reliability. Using nanowires would offer a solution to this problem.

We found that InAs and GaAs nanowires were grown perpendicular to a $\mathrm{Si}(111)$ substrate surface with reduced misfit dislocations at the interface of $\mathrm{Si}$ and the nanowire [93, 94], which demonstrated tremendous potential for developing III-V nanowire devices on $\mathrm{Si}[95,96]$. A serious issue for III- $\mathrm{V}$ nanowires on $\mathrm{Si}$ is how the series resistance attributed to the energy band offset between Si and III-V can be minimized when electric current flows across the junction interface. Using an Esaki tunnel diode structure is a solution to the problem [92]. Compatibility with the planar structure of conventional CMOS-FETs is another issue when III-V nanowires are used as channel replacements in FETs. Further ideas for structures are expected.

\section{Summary}

III-V semiconductor nanowires with heterojunctions along their axial and radial directions grown by selective-area metal organic vapor phase epitaxy were reviewed. The photoluminescence of InGaAs/GaAs, GaAs/AlGaAs, or GaAs/GaAsP was investigated in axial heterojunction nanowires to estimate the thickness of an InGaAs or GaAs single quantum well buried in them. Radial heterostructure (core-shell type heterostructure) nanowires, that is, GaAs/AlGaAs, InP/InAs/InP, or GaAs/GaAsP, were fabricated to evaluate what effect surface passivation had on the core nanowire, which indicated enhanced intensity in photoluminescence. Further, photoexcited laser emissions from a single GaAs/GaAsP core-shell nanowire were evaluated. Transmission electron microscopy combined with energy dispersive $\mathrm{X}$-ray spectroscopy was used to analyze the crystal structure and atomic composition of the nanowires, which indicated that the axial or the radial heterostructure was successfully fabricated. The results revealed the basic optical properties of the nanowires and showed their promise in applications to optoelectronics devices.

\section{Acknowledgment}

This work was financially supported by a grant-in-aid from Scientific Research from the Ministry of Education, Culture, Sports, Science and Technology (MEXT) of Japan.

\section{References}

[1] R. S. Wagner and W. C. Ellis, "Vapor-liquid-solid mechanism of single crystal growth," Applied Physics Letters, vol. 4, no. 5, pp. 89-90, 1964.

[2] G. A. Bootsma and H. J. Gassen, "A quantitative study on the growth of silicon whiskers from silane and germanium whiskers from germane," Journal of Crystal Growth, vol. 10, no. 3, pp. 223-234, 1971.

[3] E. I. Givargizov, "Fundamental aspects of VLS growth," Journal of Crystal Growth, vol. 31, pp. 20-30, 1975.
[4] J. Kasahara, K. Kajiwara, and T. Yamada, "GaAs whiskers grown by a thermal decomposition method," Journal of Crystal Growth, vol. 38, no. 1, pp. 23-28, 1977.

[5] A. M. Morales and C. M. Lieber, "A laser ablation method for the synthesis of crystalline semiconductor nanowires," Science, vol. 279, no. 5348, pp. 208-211, 1998.

[6] C. Li, D. Zhang, S. Han, X. Liu, T. Tang, and C. Zhou, "Diameter-controlled growth of single-crystalline $\mathrm{In}_{2} \mathrm{O}_{3}$ nanowires and their electronic properties," Advanced Materials, vol. 15, no. 2, pp. 143-146, 2003.

[7] W. Han, S. Fan, Q. Li, and Y. Hu, "Synthesis of gallium nitride nanorods through a carbon nanotube- confined reaction," Science, vol. 277, no. 5330, pp. 1287-1289, 1997.

[8] M. H. Huang, Y. Wu, H. Feick, N. Tran, E. Weber, and P. Yang, "Catalytic growth of zinc oxide nanowires by vapor transport," Advanced Materials, vol. 13, no. 2, pp. 113-116, 2001.

[9] K. Haraguchi, T. Katsuyama, K. Hiruma, and K. Ogawa, "GaAs p-n junction formed in quantum wire crystals," Applied Physics Letters, vol. 60, no. 6, pp. 745-747, 1992.

[10] C. Li, D. Zhang, X. Liu et al., " $\mathrm{In}_{2} \mathrm{O}_{3}$ nanowires as chemical sensors," Applied Physics Letters, vol. 82, no. 10, pp. 1613-1615, 2003.

[11] Z. L. Wang and J. Song, "Piezoelectric nanogenerators based on zinc oxide nanowire arrays," Science, vol. 312, no. 5771, pp. 243-246, 2006.

[12] B. Tian, X. Zheng, T. J. Kempa et al., "Coaxial silicon nanowires as solar cells and nanoelectronic power sources," Nature, vol. 449, no. 7164, pp. 885-889, 2007.

[13] M. T. Björk, B. J. Ohlsson, C. Thelander et al., "Nanowire resonant tunneling diodes," Applied Physics Letters, vol. 81, no. 23, pp. 4458-4460, 2002.

[14] Y. Cui, Z. Zhong, D. Wang, W. U. Wang, and C. M. Lieber, "High performance silicon nanowire field effect transistors," Nano Letters, vol. 3, no. 2, pp. 149-152, 2003.

[15] O. Gunawan and S. Guha, "Characteristics of vapor-liquidsolid grown silicon nanowire solar cells," Solar Energy Materials and Solar Cells, vol. 93, no. 8, pp. 1388-1393, 2009.

[16] A. F. i Morral, D. Spirkoska, J. Arbiol, M. Heigoldt, J. R. Morante, and G. Abstreiter, "Prismatic quantum heterostructures synthesized on molecular-beam epitaxy GaAs nanowires," Small, vol. 4, no. 7, pp. 899-903, 2008.

[17] M. Heiß, A. Gustafsson, S. Conesa-Boj et al., "Catalyst-free nanowires with axial InxGal-xAs/GaAs heterostructures," Nanotechnology, vol. 20, no. 7, Article ID 075603, 2009.

[18] B.-S. Kim, T.-W. Koo, J.-H. Lee et al., "Catalyst-free growth of single-crystal silicon and germanium nanowires," Nano Letters, vol. 9, no. 2, pp. 864-869, 2009.

[19] T. Hamano, H. Hirayama, and Y. Aoyagi, "New technique for fabrication of two-dimensional photonic bandgap crystals by selective epitaxy," Japanese Journal of Applied Physics, Part 2, vol. 36, no. 3, pp. L286-L288, 1997.

[20] J. Motohisa, J. Takeda, M. Inari, J. Noborisaka, and T. Fukui, "Growth of GaAs/AlGaAs hexagonal pillars on GaAs (111)B surfaces by selective-area MOVPE," Physica E, vol. 23, no. 3-4, pp. 298-304, 2004.

[21] M. Yoshizawa, A. Kikuchi, M. Mori, N. Fujita, and K. Kishino, "Growth of self-organized GaN nanostructures on $\mathrm{Al}_{2} \mathrm{O}_{3}(0001)$ by RF-radical source molecular beam epitaxy," Japanese Journal of Applied Physics, Part 2, vol. 36, no. 4, pp. L459-L462, 1997.

[22] M. Yoshizawa, A. Kikuchi, N. Fujita, K. Kushi, H. Sasamoto, and K. Kishino, "Self-organization of $\mathrm{GaN} / \mathrm{Al}_{0.18} \mathrm{Ga}_{0.82} \mathrm{~N}$ multi-layer nano-columns on (0001) $\mathrm{Al}_{2} \mathrm{O}_{3}$ by $\mathrm{RF}$ molecular 
beam epitaxy for fabricating GaN quantum disks," Journal of Crystal Growth, vol. 189-190, pp. 138-141, 1998.

[23] H. Sekiguchi, K. Kishino, and A. Kikuchi, "Emission color control from blue to red with nanocolumn diameter of InGaN/GaN nanocolumn arrays grown on same substrate," Applied Physics Letters, vol. 96, no. 23, Article ID 231104, 2010.

[24] W. I. Park, D. H. Kim, S.-W. Jung, and G.-C. Yi, "Metalorganic vapor-phase epitaxial growth of vertically well-aligned $\mathrm{ZnO}$ nanorods," Applied Physics Letters, vol. 80, no. 22, p. 4232, 2002.

[25] S. J. An, J. H. Chae, G.-C. Yi, and G. H. Park, "Enhanced light output of GaN-based light-emitting diodes with $\mathrm{ZnO}$ nanorod arrays," Applied Physics Letters, vol. 92, no. 12, Article ID 121108, 2008.

[26] H. Paetzelt, V. Gottschalch, J. Bauer, G. Benndorf, and G. Wagner, "Selective-area growth of GaAs and InAs nanowireshomo- and heteroepitaxy using $\mathrm{SiN}_{\mathrm{x}}$ templates," Journal of Crystal Growth, vol. 310, no. 23, pp. 5093-5097, 2008.

[27] K. Sladek, V. Klinger, J. Wensorra, M. Akabori, H. Hardtdegen, and D. Grützmacher, "MOVPE of n-doped GaAs and modulation doped GaAs/AlGaAs nanowires," Journal of Crystal Growth, vol. 312, no. 5, pp. 635-640, 2010.

[28] L. Yang, J. Motohisa, J. Takeda, K. Tomioka, and T. Fukui, "Size-dependent photoluminescence of hexagonal nanopillars with single InGaAs/GaAs quantum wells fabricated by selective-area metal organic vapor phase epitaxy," Applied Physics Letters, vol. 89, no. 20, Article ID 203110, 2006.

[29] J. Noborisaka, J. Motohisa, S. Hara, and T. Fukui, "Fabrication and characterization of freestanding GaAs/AlGaAs core-shell nanowires and AlGaAs nanotubes by using selective-area metalorganic vapor phase epitaxy," Applied Physics Letters, vol. 87, no. 9, pp. 1-3, 2005.

[30] A. Hayashida, T. Sato, S. Hara, J. Motohisa, K. Hiruma, and T. Fukui, "Fabrication and characterization of GaAs quantum well buried in AlGaAs/GaAs heterostructure nanowires," Journal of Crystal Growth, vol. 312, no. 24, pp. 3592-3598, 2010.

[31] P. Mohan, J. Motohisa, and T. Fukui, "Fabrication of InPInAsInP core-multishell heterostructure nanowires by selective area metalorganic vapor phase epitaxy," Applied Physics Letters, vol. 88, no. 13, Article ID 133105, 2006.

[32] B. Hua, J. Motohisa, Y. Kobayashi, S. Hara, and T. Fukui, "Single GaAs/GaAsP coaxial core-shell nanowire lasers," Nano Letters, vol. 9, no. 1, pp. 112-116, 2009.

[33] S. Fujisawa, T. Sato, S. Hara, J. Motohisa, K. Hiruma, and T. Fukui, "Growth and characterization of a GaAs quantum well buried in $\mathrm{GaAsP} / \mathrm{GaAs}$ vertical heterostructure nanowires by selective-area metal organic vapor phase epitaxy," Japanese Journal of Applied Physics, vol. 50, no. 4, part 2, article 04DH03, 2011.

[34] D. K. Biegelsen, R. D. Bringans, J. E. Northrup, and L.-E. Swartz, "Reconstructions of GaAs(111) surfaces observed by scanning tunneling microscopy," Physical Review Letters, vol. 65, no. 4, pp. 452-455, 1990.

[35] M. Yoshimura, K. Tomioka, K. Hiruma, S. Hara, J. Motohisa, and T. Fukui, "Growth and characterization of InGaAs nanowires formed on GaAs(111)B by selective-area metal organic vapor phase epitaxy," Japanese Journal of Applied Physics, vol. 49, no. 4, Article ID 04DH08, 2010.

[36] T. Nishida, K. Uwai, Y. Kobayashi, and N. Kobayashi, "Phase diagram of GaAs (111)B surface during metal-organic chemical vapor deposition measured by surface photo-absorption,"
Japanese Journal of Applied Physics, Part 1, vol. 34, no. 12, pp. 6326-6330, 1995.

[37] J. Noborisaka, J. Motohisa, and T. Fukui, "Catalyst-free growth of GaAs nanowires by selective-area metalorganic vapor-phase epitaxy," Applied Physics Letters, vol. 86, no. 21, Article ID 213102, pp. 1-3, 2005.

[38] P. Bhattacharya, Properties of Lattice-Matched and Strained Indium Gallium Arsenide, EMIS Data Reviews Series No. 8, IE, London, UK, 1993.

[39] Y. D. Galeuchet, P. Roentgen, and V. Graf, "GaInAs/InP selective area metalorganic vapor phase epitaxy for one-stepgrown buried low-dimensional structures," Journal of Applied Physics, vol. 68, no. 2, pp. 560-568, 1990.

[40] T. Takebe, M. Fujii, T. Yamamoto, K. Fujita, and T. Watanabe, "Orientation-dependent Ga surface diffusion in molecular beam epitaxy of GaAs on GaAs patterned substrates," Journal of Applied Physics, vol. 81, no. 11, pp. 7273-7281, 1997.

[41] T. Sasaki, M. Kitamura, and I. Mito, "Selective metalorganic vapor phase epitaxial growth of InGaAsP / InP layers with bandgap energy control in InGaAs/InGaAsP multiplequantum well structures," Journal of Crystal Growth, vol. 132, no. 3-4, pp. 435-443, 1993.

[42] S. Martini, A. A. Quivy, A. Tabata, and J. R. Leite, "Influence of the temperature and excitation power on the optical properties of InGaAs/GaAs quantum wells grown on vicinal GaAs(001) surfaces," Journal of Applied Physics, vol. 90, no. 5, pp. 22802289, 2001.

[43] L. Yang, J. Motohisa, J. Takeda, K. Tomioka, and T. Fukui, "Selective-area growth of hexagonal nanopillars with single InGaAs/GaAs quantum wells on GaAs(111)B substrate and their temperature-dependent photoluminescence," Nanotechnology, vol. 18, no. 10, Article ID 105302, 2007.

[44] L. Ouattara, A. Mikkelsen, N. Sköld et al., "GaAs/AlGaAs nanowire heterostructures studied by scanning tunneling microscopy," Nano Letters, vol. 7, no. 9, pp. 2859-2864, 2007.

[45] K. Ishikawa, N. Yamamoto, K. Tateno, and Y. Watanabe, "Characterization of individual GaAs/AlGaAs self-standing nanowires by cathodoluminescence technique using transmission electron microscope," Japanese Journal of Applied Physics, Part 1, vol. 47, no. 8, pp. 6596-6600, 2008.

[46] C. Chen, N. Braidy, C. Couteau, C. Fradin, G. Weihs, and R. Lapierre, "Multiple quantum well AlGaAs nanowires," Nano Letters, vol. 8, no. 2, pp. 495-499, 2008.

[47] J. Heinrich, A. Huggenberger, T. Heindel et al., "Single photon emission from positioned GaAs/AlGaAs photonic nanowires," Applied Physics Letters, vol. 96, no. 21, Article ID 211117, 2010.

[48] M. Nakayama, T. Hirao, and T. Hasegawa, "Characteristics of photoluminescence due to exciton-exciton scattering in GaAs/AlAs multiple quantum wells," Journal of Applied Physics, vol. 105, no. 12, Article ID 123525, 2009.

[49] R. Dingle, W. Wiegmann, and C. H. Henry, "Quantum states of confined carriers in very thin $\mathrm{Al}_{\mathrm{x}} \mathrm{Ga}_{1-\mathrm{x}} \mathrm{As}-\mathrm{GaAs}_{\mathrm{A}} \mathrm{Al}_{\mathrm{x}} \mathrm{Ga}_{1-\mathrm{x}} \mathrm{As}$ heterostructures," Physical Review Letters, vol. 33, no. 14, pp. 827-830, 1974.

[50] S. M. Sze, Physics of Semiconductor Devices, John Wiley \& Sons, New York, NY, USA, 2nd edition, 1981.

[51] M. J. Tambe, F. Allard, and S. Gradĉeak, "Characterization of core-shell GaAs/AlGaAs nanowire heterostructures using advanced electron microscopy," Journal of Physics: Conference Series, no. 209, Article ID 012033, 2010.

[52] P. K. Mohseni, C. Maunders, G. A. Botton, and R. R. LaPierre, "GaP/GaAsP/GaP core-multishell nanowire heterostructures 
on (111) silicon," Nanotechnology, vol. 18, no. 44, Article ID 445304, 2007.

[53] C. Chen, M. C. Plante, C. Fradin, and R. R. LaPierre, "Layerby-layer and step-flow growth mechanisms in $\mathrm{GaAsP} / \mathrm{GaP}$ nanowire heterostructures," Journal of Materials Research, vol. 21, no. 11, pp. 2801-2809, 2006.

[54] C. P. T. Svensson, W. Seifert, M. W. Larsson et al., "Epitaxially grown $\mathrm{GaP} / \mathrm{GaAs} 1-\mathrm{xPx} / \mathrm{GaP}$ double heterostructure nanowires for optical applications," Nanotechnology, vol. 16, no. 6, pp. 936-939, 2005.

[55] W. Seifert, M. Borgström, K. Deppert et al., "Growth of onedimensional nanostructures in MOVPE," Journal of Crystal Growth, vol. 272, no. 1-4, pp. 211-220, 2004.

[56] J. J. Tietjen and J. A. Amick, "The preparation and properties of vapor-deposited epitaxial $\mathrm{GaAs}_{1-\mathrm{x}} \mathrm{P}_{\mathrm{x}}$ using arsine and phosphine," Journal of the Electrochemical Society, vol. 113, no. 7, pp. 724-728, 1966.

[57] A. P. Roth, S. Charbonneau, and R. G. Goodchild, "Residual shallow acceptors in GaAs layers grown by metal-organic vapor phase epitaxy," Journal of Applied Physics, vol. 54, no. 9, pp. 5350-5357, 1983.

[58] S. Ando, S. S. Chang, and T. Fukui, "Selective epitaxy of GaAs/AlGaAs on (111) B substrates by MOCVD and applications to nanometer structures," Journal of Crystal Growth, vol. 115, no. 1-4, pp. 69-73, 1991.

[59] S. Kodama, S. Koyanagi, T. Hashizume, and H. Hasegawa, "Silicon interlayer based surface passivation of near-surface quantum wells," Journal of Vacuum Science and Technology B, vol. 13, no. 4, pp. 1794-1800, 1995.

[60] J. M. Moison, K. Elcess, F. Houzay et al., "Near-surface GaAs $/ \mathrm{Ga}_{0.7} \mathrm{Al}_{0.3}$ As quantum wells: interaction with the surface states," Physical Review B, vol. 41, no. 18, pp. 12945-12948, 1990.

[61] H. W. Seo, S. Y. Bae, J. Park, H. Yang, K. S. Park, and S. Kim, "Strained gallium nitride nanowires," Journal of Chemical Physics, vol. 116, no. 21, pp. 9492-9499, 2002.

[62] L. J. Lauhon, M. S. Gudlksen, D. Wang, and C. M. Lieber, "Epitaxial core-shell and core-multishell nanowire heterostructures," Nature, vol. 420, no. 6911, pp. 57-61, 2002.

[63] F. Qian, Y. Li, S. Gradečak, D. Wang, C. J. Barrelet, and C. M. Lieber, "Gallium nitride-based nanowire radial heterostructures for nanophotonics," Nano Letters, vol. 4, no. 10, pp. 1975-1979, 2004.

[64] F. Qian, S. Gradečak, Y. Li, C.-Y. Wen, and C. M. Lieber, "Core/multishell nanowire heterostructures as multicolor, high-efficiency light-emitting diodes," Nano Letters, vol. 5, no. 11, pp. 2287-2291, 2005.

[65] P. Mohan, J. Motohisa, and T. Fukui, "Controlled growth of highly uniform, axial/radial direction-defined, individually addressable InP nanowire arrays," Nanotechnology, vol. 16, no. 12, pp. 2903-2907, 2005.

[66] P. Mohan, J. Motohisa, and T. Fukui, "Realization of conductive InAs nanotubes based on lattice-mismatched InP/InAs core-shell nanowires," Applied Physics Letters, vol. 88, no. 1, Article ID 013110, 2006.

[67] S. Hara, J. Motohisa, J. Noborisaka, J. Takeda, and T. Fukui, "Photoluminescence from single hexagonal nano-wire grown by selective area MOVPE," Institute of Physics Conference Series, no. 184, section 5, pp. 393-398, 2005.

[68] C. G. Van de Walle, "Band lineups and deformationpotentials in the model-solid theory," Physical Review B, vol. 39, no. 3, pp. 1871-1883, 1989.
[69] Y. Kajikawa, M. Hata, and T. Isu, "Optical matrix elements in (110)-oriented quantum wells," Japanese Journal of Applied Physics, Part 1, vol. 30, no. 9, pp. 1944-1945, 1991.

[70] S. L. Chuang, "Efficient band-structure calculations of strained quantum wells," Physical Review B, vol. 43, no. 12, pp. 9649-9661, 1991.

[71] M. Sugawara, N. Okazaki, T. Fujii, and S. Yamazaki, "Conduction-band and valence-band structures in strained $\mathrm{In}_{1-\mathrm{x}} \mathrm{Ga}_{\mathrm{x}} \mathrm{As} / \mathrm{InP}$ quantum wells on (001) InP substrates," Physical Review B, vol. 48, no. 11, pp. 8102-8118, 1993.

[72] B. Pal, K. Goto, M. Ikezawa et al., "Type-II behavior in wurtzite InP/InAs/InP core-multishell nanowires," Applied Physics Letters, vol. 93, no. 7, Article ID 073105, 2008.

[73] X. Duan, Y. Huang, R. Agarwal, and C. M. Lieber, "Singlenanowire elecctrically driven lasers," Nature, vol. 421, no. 6920, pp. 241-245, 2003.

[74] M. H. Huang, S. Mao, H. Feick et al., "Room-temperature ultraviolet nanowire nanolasers," Science, vol. 292, no. 5523, pp. 1897-1899, 2001.

[75] J. C. Johnson, H.-J. Choi, K. P. Knutsen, R. D. Schaller, P. Yang, and R. J. Saykally, "Single gallium nitride nanowire lasers," Nature Materials, vol. 1, no. 2, pp. 106-110, 2002.

[76] H.-J. Choi, J. C. Johnson, R. He et al., "Self-organized GaN quantum wire UV lasers," Journal of Physical Chemistry B, vol. 107, no. 34, pp. 8721-8725, 2003.

[77] S. Gradečak, F. Qian, Y. Li, H.-G. Park, and C. M. Lieber, "GaN nanowire lasers with low lasing thresholds," Applied Physics Letters, vol. 87, no. 17, Article ID 173111, pp. 1-3, 2005.

[78] B. Cao, Y. Jiang, C. Wang et al., "Synthesis and lasing properties of highly ordered CdS nanowire arrays," Advanced Functional Materials, vol. 17, no. 9, pp. 1501-1508, 2007.

[79] F. Qian, Y. Li, S. Gradečak et al., "Multi-quantum-well nanowire heterostructures for wavelength-controlled lasers," Nature Materials, vol. 7, no. 9, pp. 701-706, 2008.

[80] A. H. Chin, S. Vaddiraju, A. V. Maslov, C. Z. Ning, M. K. Sunkara, and M. Meyyappan, "Near-infrared semiconductor subwavelength-wire lasers," Applied Physics Letters, vol. 88, no. 16, Article ID 163115, 2006.

[81] K. Ikejiri, T. Sato, H. Yoshida et al., "Growth characteristics of GaAs nanowires obtained by selective area metal-organic vapour-phase epitaxy," Nanotechnology, vol. 19, no. 26, Article ID 265604, 2008.

[82] B. Hua, J. Motohisa, Y. Ding, S. Hara, and T. Fukui, "Characterization of Fabry-Pérot microcavity modes in GaAs nanowires fabricated by selective-area metal organic vapor phase epitaxy," Applied Physics Letters, vol. 91, no. 13, Article ID 131112, 2007.

[83] J. C. Johnson, H. Yan, P. Yang, and R. J. Saykally, "Optical cavity effects in $\mathrm{ZnO}$ nanowire lasers and waveguides," Journal of Physical Chemistry B, vol. 107, no. 34, pp. 8816-8828, 2003.

[84] P. J. Pauzauskie, D. J. Sirbuly, and P. Yang, "Semiconductor nanowire ring resonator laser," Physical Review Letters, vol. 96, no. 14, Article ID 143903, pp. 1-4, 2006.

[85] K. Ogawa, K. Hiruma, and T. Katsuyama, "Photoluminescence characteristics of GaAs nanowhiskers: effects of depletion potential," The Institute of Electronics, Information and Communication Engineers Transactions on Electronics, vol. E79-C, no. 11, pp. 1573-1578, 1996.

[86] M. H. M. van Weert, O. Wunnicke, A. L. Roest et al., "Large redshift in photoluminescence of $p$-doped InP nanowires induced by Fermi-level pinning," Applied Physics Letters, vol. 88, no. 4, Article ID 043109, pp. 1-3, 2006. 
[87] L. K. van Vugt, S. Ruhle, and D. Vanmaekelbergh, "Phasecorrelated nondirectional laser emission from the end facets of a ZnO nanowire," Nano Letters, vol. 6, no. 12, pp. 2707-2711, 2006.

[88] T. Mårtensson, C. Patrik, T. Svensson et al., "Epitaxial III-V nanowires on silicon," Nano Letters, vol. 4, no. 10, pp. 19871990, 2004.

[89] B. Mandl, J. Stangl, T. Mårtensson et al., "Au-free epitaxial growth of InAs nanowires," Nano Letters, vol. 6, no. 8, pp. 1817-1821, 2006.

[90] T. Mårtensson, J. B. Wagner, E. Hilner et al., "Epitaxial growth of indium arsenide nanowires on silicon using nucleation templates formed by self-assembled organic coatings," Advanced Materials, vol. 19, no. 14, pp. 1801-1806, 2007.

[91] M. Moewe, L. C. Chuang, S. Crankshaw, C. Chase, and C. Chang-Hasnain, "Atomically sharp catalyst-free wurtzite GaAsAlGaAs nanoneedles grown on silicon," Applied Physics Letters, vol. 93, no. 2, Article ID 023116, 2008.

[92] M. T. Björk, H. Schmid, C. D. Bessire et al., "Si-InAs heterojunction Esaki tunnel diodes with high current densities," Applied Physics Letters, vol. 97, no. 16, Article ID 163501, 2010.

[93] K. Tomioka, J. Motohisa, S. Hara, and T. Fukui, "Control of InAs nanowire growth directions on Si," Nano Letters, vol. 8, no. 10, pp. 3475-3480, 2008.

[94] K. Tomioka, Y. Kobayashi, J. Motohisa, S. Hara, and T. Fukui, "Selective-area growth of vertically aligned GaAs and GaAs/AlGaAs core-shell nanowires on $\mathrm{Si}(111)$ substrate," Nanotechnology, vol. 20, no. 14, Article ID 145302, 2009.

[95] T. Tanaka, K. Tomioka, S. Hara, J. Motohisa, E. Sano, and T. Fukui, "Vertical surrounding gate transistors using single InAs nanowires grown on Si substrates," Applied Physics Express, vol. 3, no. 2, Article ID 025003, 2010.

[96] K. Tomioka, J. Motohisa, S. Hara, K. Hiruma, and T. Fukui, "GaAs/AlGaAs core multishell nanowire-based light-emitting diodes on Si," Nano Letters, vol. 10, no. 5, pp. 1639-1644, 2010. 

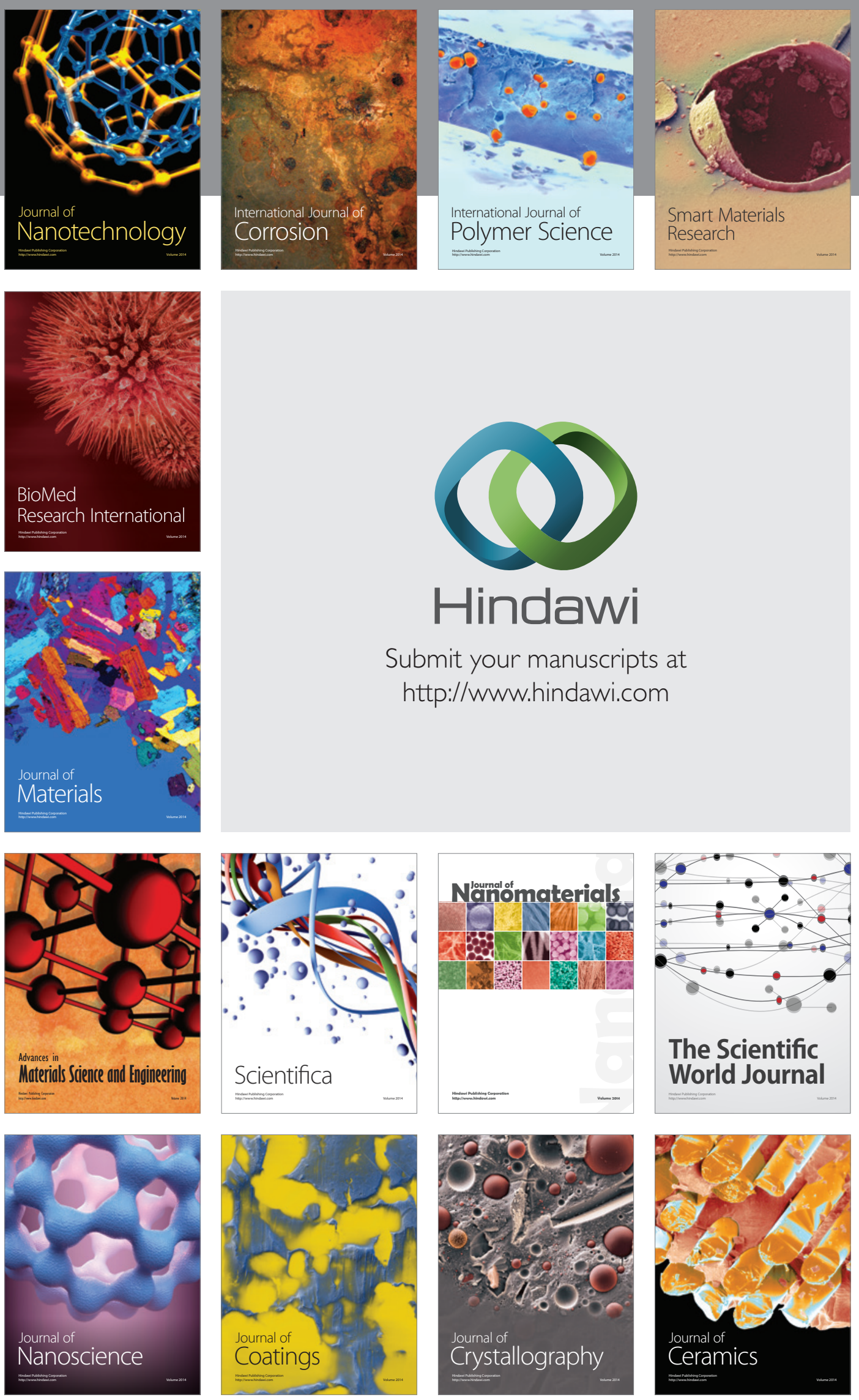

The Scientific World Journal

Submit your manuscripts at

http://www.hindawi.com

\section{World Journal}

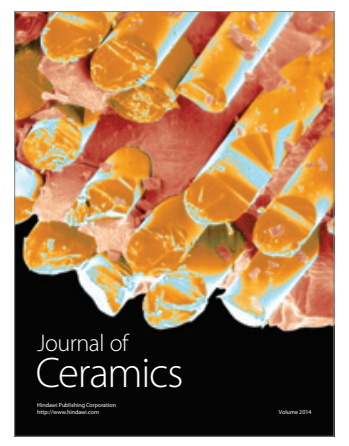

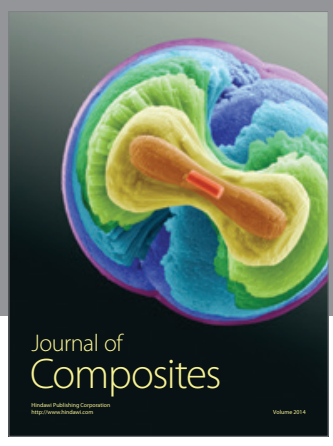
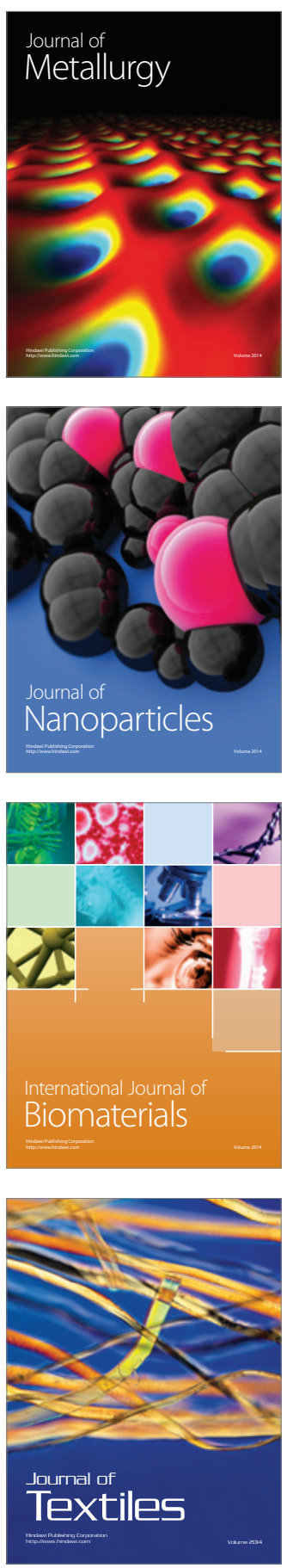